\title{
HIGHLY VARIABLE EXTINCTION AND ACCRETION IN THE JET-DRIVING CLASS I-TYPE YOUNG STAR PTF 10nvg (V2492 Cyg, IRAS 20496+4354)
}

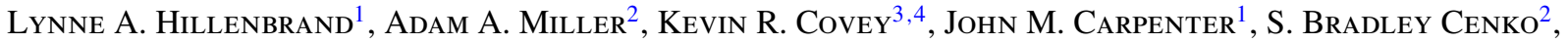 \\ JefFrey M. Silverman ${ }^{2}$, Philip S. Muirhead ${ }^{1}$, William J. Fischer ${ }^{5}$, Justin R. CrepP ${ }^{1,6}$, \\ Joshua S. Bloom ${ }^{2}$, AND Alexei V. FilipPEnKo ${ }^{2}$ \\ ${ }^{1}$ Astronomy Department, California Institute of Technology, Pasadena, CA 91125, USA \\ ${ }^{2}$ Department of Astronomy, University of California, Berkeley, CA 94720-3411, USA \\ ${ }^{3}$ Department of Astronomy, Cornell University, 226 Space Sciences Building, Ithaca, NY 14853, USA \\ ${ }^{4}$ Lowell Observatory, 1400 West Mars Hill Road, Flagstaff, AZ 86001, USA \\ ${ }^{5}$ Department of Physics and Astronomy, University of Toledo, 2801 West Bancroft Street, Toledo, OH 43606, USA \\ Received 2012 July 31; accepted 2012 December 7; published 2013 January 30
}

\begin{abstract}
We report extensive new photometry and spectroscopy of the highly variable young stellar object PTF 10nvg (also known as IRAS 20496+4354 and V2492 Cyg), including optical and near-infrared time-series data as well as mid-infrared and millimeter data. Following the previously reported 2010 rise to $R_{\mathrm{PTF}} \lesssim 13 \cdot 5$ and subsequent fade, during 2011 and 2012 the source underwent additional episodes of brightening, followed by several magnitude dimming events including prolonged faint states at $R_{\text {PTF }} \gtrsim 20^{\mathrm{m}}$. The observed high-amplitude variations are largely consistent with extinction changes $\left(\Delta A_{V}\right.$ up to $30 \mathrm{mag}$ ) having a $\sim 220$ day quasi-periodic signal. However, photometry measured when the source was near maximum brightness in mid-2010 as well as in late-2012 does not phase well to this period. Spectral evolution includes not only changes in the spectral slope but also correlated variation in the prominence of $\mathrm{TiO} / \mathrm{VO} / \mathrm{CO}$ bands and atomic line emission, as well as anti-correlated variation in forbidden line emission which, along with $\mathrm{H}_{2}$, dominates optical and infrared spectra at faint epochs. Notably, nightto-night variations in several forbidden doublet strengths and ratios are observed. High-dispersion spectra were obtained in a variety of photometric states and reveal time-variable line profiles. Neutral and singly ionized atomic species are likely formed in an accretion flow and/or impact while the origin of zero-velocity atomic Li I $\lambda 6707$ in emission is unknown. Forbidden lines, including several rare species, exhibit blueshifted emission profiles and likely arise from an outflow/jet. Several of these lines are also seen spatially offset from the continuum source position, presumably in a shocked region of an extended jet. Blueshifted absorption components of the Na I D doublet, $\mathrm{K}_{\mathrm{I}} \lambda \lambda 7665,7669$ doublet, and the O I 7774 triplet, as well as blueshifted absorption components seen against the broad $\mathrm{H} \alpha$ and $\mathrm{Ca}$ II triplet emission lines, similarly are formed in the outflow. CARMA maps resolve on larger scales a spatially extended outflow in millimeter-wavelength $\mathrm{CO}$. We attribute the recently observed photometric and spectroscopic behavior to rotating circumstellar disk material located at separation $a \approx 0.7\left(M_{*} / M_{\odot}\right)^{1 / 3} \mathrm{AU}$ from the continuum source, causing the semi-periodic dimming. Occultation of the central star as well as the bright inner disk and the accretion/outflow zones renders shocked gas in the inner part of the jet amenable to observation at the faint epochs. We discuss PTF 10nvg as a source exhibiting both accretion-driven (perhaps analogous to V1647 Ori) and extinction-driven (perhaps analogous to UX Ori or GM Cep) high-amplitude variability phenomena.
\end{abstract}

Key words: stars: formation - stars: individual (V2492 Cyg, PTF 10nvg, IRAS 20496+4354) - stars: pre-main sequence

Online-only material: color figures, machine-readable and VO tables

\section{INTRODUCTION}

PTF 10nvg, in the North America Nebula region of recent star formation, was identified by the Palomar Transient Factory (PTF; Law et al. 2009; Rau et al. 2009) on 2010 July 8 as an optical transient based on automatic discovery and classification codes (Bloom et al. 2012). The source was rapidly followed up by the PTF collaboration. We announced our findings concerning the 2010 outburst of this Class I-type young star in Covey et al. (2011) where we presented optical and infrared light curves and multi-epoch optical and infrared spectroscopy. Therein, we made the analogy of PTF 10nvg (now called V2492 Cyg) to the behavior of V1647 Ori, an embedded Class I-type young star that was observed over the past decade to undergo several large-amplitude photometric events on few-year timescales.

\footnotetext{
6 Current address: Department of Physics, University of Notre Dame, 225 Nieuwland Science Hall, Notre Dame, IN 46556, USA.
}

V1647 Ori and PTF 10nvg both displayed 4-6 mag photometric rises from their faint states that were similar in the early stages to the outbursts of FU Ori stars. However, these particular objects and others like them do not have the spectroscopic characteristics of FU Ori stars; rather than being absorption line dominated (especially at high dispersion), they are emission line dominated, with absorption seen in only a handful of blueshifted features arising in strong winds. Further, the V1647 Ori-type objects do not remain in the elevated photometric state for the long timescale associated with FU Ori outbursts (estimated at roughly a century); instead, their brightening episodes last only a few months to a few years and are characterized by large-amplitude fluctuations on month to few month timescales. Members of this category possibly undergo repeated episodes of their outbursting (and/or extinction-dominated) behavior at several year intervals. In this regard they are similar to the lower amplitude ( 2-4 mag) but repeating outbursts of EX Lup-type systems, which last months to $>1$ year each and repeat on few-year to decade intervals. 
Whether small-scale, low-amplitude events such as EX Luptype outbursts, larger scale, high-amplitude events such as the V1647 Ori-type events, or similarly large-amplitude but also longer duration FU Ori-type events, the star/disk system in such outbursts is interpreted as undergoing an episode of enhanced mass accretion and associated mass outflow. The accretion mechanisms for the different categories of objects are likely related, and are attributed to instabilities in the inner accretion disks, possibly associated with cyclic magnetospheredisk interactions. For PTF 10nvg in particular, Covey et al. (2011) estimated a mass accretion rate of $2.5 \times 10^{-7} M_{\odot} \mathrm{yr}^{-1}$ in the elevated state of $2010,{ }^{7}$ similar to the value estimated for EX Lup during its 2008 outburst (e.g., Juhász et al. 2012).

Time-variable accretion, however, may not be the entire explanation for many large-amplitude young star variables. As demonstrated herein, time-variable extinction is clearly an important part of the PTF 10nvg interpretation, and may also play a significant role in the observed photometric behavior of many of the so-called outburst light curves highlighted to date in the literature. For example, GM Cep was discussed by SiciliaAguilar et al. (2008) as an EX Lup-type object, but later assessed by Xiao et al. (2010), Semkov \& Peneva (2012), and Chen et al. (2012) as having extinction-dominated rather than accretiondominated time-series behavior. Both phenomena are perhaps simultaneously relevant, as we argue here for PTF 10nvg. Other well-known low-amplitude young star variables such as UX Ori, RR Tau, VV Ser, AA Tau as well as the "dippers" discussed by Morales-Calderón et al. (2011) and Cody \& Hillenbrand (2010, 2011) also appear to be undergoing short timescale extinction events. Larger amplitude and-importantly-longperiod examples include KH15D (Hamilton et al. 2012), WL4 (Plavchan et al. 2008), and YLW16A (Plavchan et al. 2010), which have found explanation in binary interactions with circumstellar disk material.

The 2010 brightening of PTF 10nvg was independently detected by K. Itagaki and reported in 2010 August in the subscription service of the Central Bureau for Astronomical Telegrams as CBET 2426. Additional papers to date discussing the post-outburst source include those by Kóspál et al. (2011) and Aspin (2011) who also present multi-color photometric, spectroscopic, and spectral energy distribution (SED) analysis of the source, often using the Covey et al. (2011) data.

In this paper, we present new data gathered by us since the publication of Covey et al. (2011). Intensive time-series photometry shows that the source has continued its large-amplitude and color photometric fluctuations. Time-series spectroscopy demonstrates (1) continuum changes; (2) variation in the broad $\mathrm{TiO} / \mathrm{VO}$ optical band emission that was detected for the first time during the 2010 outburst of PTF 10nvg, along with variation in other molecules such as $\mathrm{CO}$ and $\mathrm{H}_{2}$; and (3) drastic changes in the atomic emission lines indicative of accretion and outflow that are correlated (permitted lines) and anti-correlated (forbidden lines) with the photometric brightness. We also present highdispersion spectral data; the line profiles are used to quantify the velocities relevant to the inflowing and outflowing material. An updated post-outburst SED is discussed, demonstrating that the source brightened relative to its historical SED in the mid-infrared, as well as in the near-infrared and optical, during 2010. A spatially extended, low-velocity molecular outflow is detected at millimeter wavelengths. New high spatial resolution near-infrared imaging rules out the presence of stellar companions within several hundred AU.

The accretion rates quoted in Aspin (2011) are a factor of 2.5-10 higher.
In Section 2, we describe our observations from 2009 to 2012. We then present our analysis of the multi-wavelength light curves (Section 3), changes in the overall spectral energy distribution (Section 4), and analysis of the continuum, absorption line, and emission line spectroscopy including discussion of spatially offset forbidden line emission in Section 5. In Section 6, we present constraints on the source multiplicity from high angular resolution direct imaging. In Section 7, we interpret our results and discuss the broader implications of our findings for accretion and extinction evolution of young stars. Finally, in Section 8, we summarize and conclude.

\section{OBSERVATIONS, DATA REDUCTION, AND RESULTS}

Our photometric and spectroscopic monitoring of PTF 10nvg (V2492 Cyg) between 2009 and 2012 includes continued monitoring in the $R$-band as well as $J$-, $H$-, and $K_{s}$-band time-series photometry. Almost 30 epochs of low-dispersion spectroscopy at optical or infrared wavelengths were obtained. High-dispersion spectroscopy in the red optical was acquired at six epochs and in the $1 \mu \mathrm{m}$ atmospheric window at three epochs. In this section, we describe the details of these various observations and resulting data sets.

\subsection{New Photometry}

\subsubsection{Optical Data from the Palomar Transient Factory}

Continued optical monitoring of PTF 10nvg was conducted as part of the regular observations of the greater North America Nebula field with the main PTF Survey Camera on the 48 inch Samuel Oschin Telescope at Palomar Observatory (hereafter P48). All observations were taken with $60 \mathrm{~s}$ exposures in the $R_{\mathrm{PTF}}$ filter, a Mould $R$ filter that is similar to the SDSS$r$ band (see Law et al. 2009). P48 observations from 2009 August to 2010 December are presented in Covey et al.; the new observations reported here started in 2011 February and continued through 2012 October. The typical cadence during the 2011 observing season was two observations separated by roughly one hour obtained every night during astronomical bright time, though there was a period of high-cadence observations during 2011 July with as many as eight observations taken in a single night. From 2012 April 18 onward, a typical cadence of three observations per night was adopted.

Brightness variations of PTF 10nvg are measured via a pointspread function (PSF) fitting routine as described in Sullivan et al. (2011). Briefly, in each image frame the PSF is determined from several field stars and the average PSF is then fit to the position of PTF 10nvg with each pixel weighted according to photon statistics. Saturated pixels are masked from the fit, enabling photometry on even moderately saturated point sources (relevant near the absolute peak in 2010). The flux calibration is performed relative to Sloan Digital Sky Survey (SDSS), based on nightly observations of SDSS calibration fields (see Ofek et al. 2012 for further details), and all reported magnitudes are given for the native $R_{\mathrm{PTF}}$ filter in the Vega system. ${ }^{8}$ Finally, we note that while the photometric measurements presented here are consistent with those we previously published in Covey et al., the improved calibration and PSF model used here supersede our previous photometry.

The optical light curve for PTF 10nvg is shown in Figure 1 and photometric measurements are reported in Table 1. From 2011

\footnotetext{
8 We convert from the $\mathrm{AB}$ mag system to the Vega system using the following transformation: $m_{R}($ Vega $)=m_{R}(\mathrm{AB})-0.19$.
} 


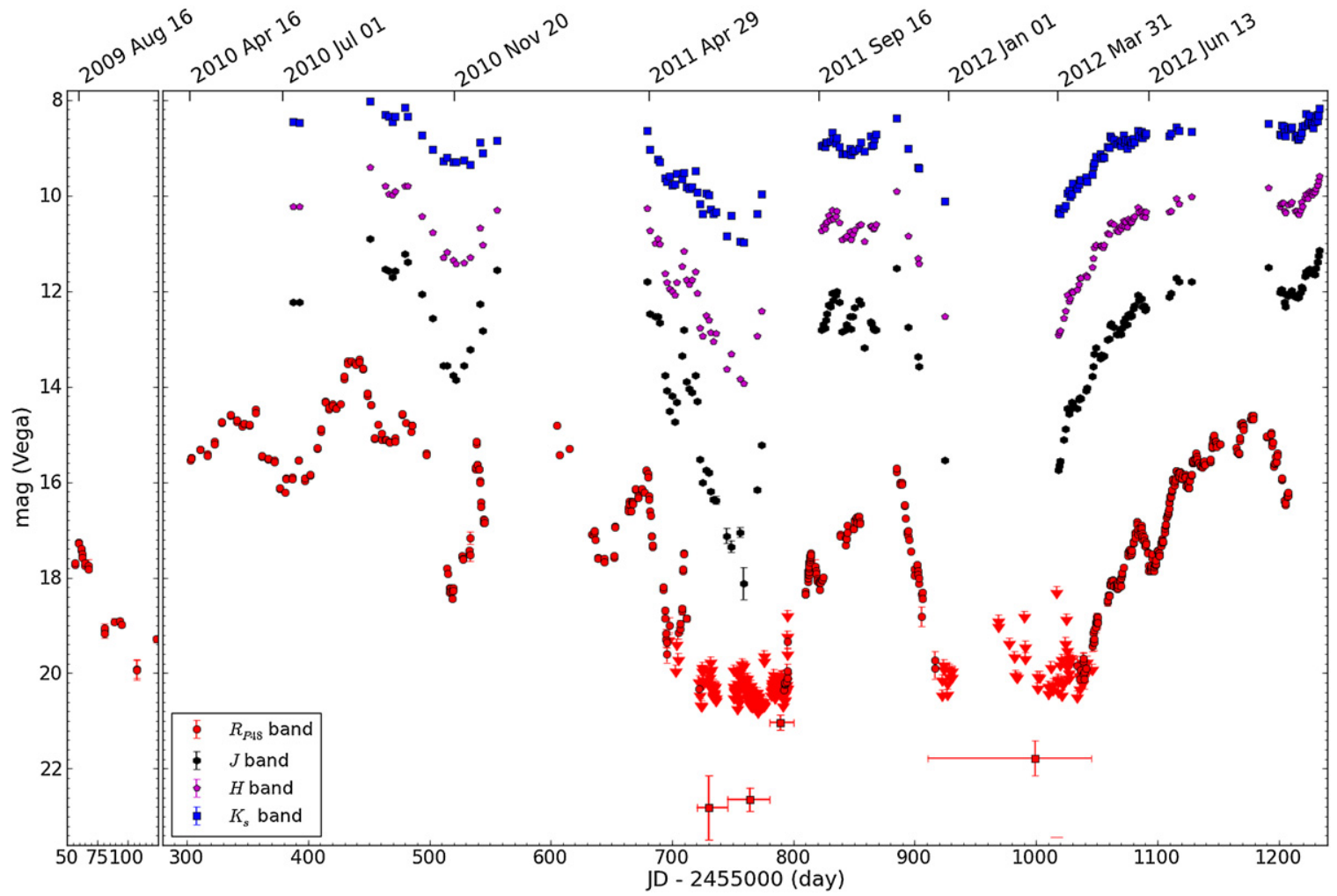

Figure 1. Multi-wavelength light curve of PTF 10nvg with UT dates indicated above the figure. From bottom to top the data streams represent variability in the $R$ band (red; data from PTF) and in the $J, H$, and $K$ bands (black, purple, and blue respectively; data from PAIRITEL). Error bars are shown, but the uncertainty in magnitude is typically smaller than the size of the symbols. During faint states when the source was not detected in individual frames, photometry was measured from stacked PTF images (red squares, in the 21-23 mag range); horizontal error bars indicate the time range of measurements included in each stack.

(A color version of this figure is available in the online journal.)

Table 1

P48 R-band Photometry of PTF 10nvg

\begin{tabular}{lcc}
\hline \hline Epoch (MJD) & $\begin{array}{c}R_{\text {PTF }} \\
(\mathrm{mag})\end{array}$ & err \\
\hline 55056.221 & 17.831 & \\
55056.295 & 17.772 & 0.019 \\
55059.262 & 17.328 & 0.020 \\
55059.357 & 17.380 & 0.017 \\
55061.316 & 17.520 & 0.017 \\
$\ldots$ & $\ldots$ & 0.023 \\
$\ldots$ & $\ldots$ & $\ldots$ \\
$\ldots$ & $\ldots$ & $\ldots$ \\
$\ldots$ & $\ldots$ & $\ldots$ \\
$\ldots$ & $\ldots$ & $\ldots$ \\
$\ldots$ & $\ldots$ & $\ldots$ \\
$\ldots$ & $\ldots$ & $\ldots$ \\
\hline
\end{tabular}

(This table is available in its entirety in machine-readable and Virtual Observatory (VO) forms in the online journal. A portion is shown here for guidance regarding its form and content.)

June 8 through 2011 August 21, and 2011 December 26 to 2012 April 9, PTF 10nvg was below the $5 \sigma$ detection limit of the P48 images. In order to constrain the brightness of PTF 10nvg during these prolonged faint periods, we took the average flux from the PTF measurements over $\sim 1$ month intervals and measured timeaveraged photometry. Uncertainties were determined by adding in quadrature the flux uncertainties from the individual nondetections and dividing by the total number of images included in the average flux measurement. The averages and the dates over which they were measured are shown in Figure 1 and summarized in Table 2. During the summer of 2011 PTF 10nvg was on average $R \sim 23 \mathrm{mag}$, which can be compared to its summer of 2010 peak brightness $R \sim 13.5 \mathrm{mag}$.

\subsubsection{Near-infrared Data from PAIRITEL}

Continued near-infrared monitoring of PTF 10nvg was conducted with the $1.3 \mathrm{~m}$ Peters Automated Infrared Imaging Telescope (PAIRITEL; Bloom et al. 2006) on Mt. Hopkins, AZ. PAIRITEL is a robotic system using the former Two Micron All Sky Survey (2MASS) Southern Hemisphere survey camera that employs two dichroics to observe simultaneously in the $J, H$, and $K_{s}$ bands. Observations were scheduled and executed via a robotic system. PAIRITEL is operated in a fixed observing mode in which $7.8 \mathrm{~s}$ double-correlated images are created from the difference of a $7.851 \mathrm{~s}$ and a $51 \mathrm{~ms}$ integration taken in rapid succession (see Blake et al. 2008). The standard observing procedure involves taking three image pairs prior to dithering the telescope.

The raw data from these images are reduced using standard IR reduction methods via PAIRITEL PIPELINE III and the flux for all sources is measured via aperture photometry using SExtractor (Bertin \& Arnouts 1996). The final absolute calibration is determined using high signal-to-noise detections from 2MASS. When PTF 10nvg is very bright, corresponding to roughly $m<10 \mathrm{mag}$, it saturates the $7.851 \mathrm{~s}$ frames. This occurs in most of the reported $K_{s}$-band imaging and at some of the brightest epochs also in the $H$ band. PIPELINE III produces "short-frame" mosaics consisting of reduced, stacked $51 \mathrm{~ms}$ images that are used for bright stage photometry (see Bloom 
Table 2

Stacked $R_{\text {PTF }}$ Photometry Representing Faint Epochs

\begin{tabular}{lcccc}
\hline \hline Mean Epoch (MJD) & $\begin{array}{c}R_{\text {PTF }} \\
(\mathrm{mag})\end{array}$ & err & Start of Stack (MJD) & End of Stack (MJD) \\
\hline 55729.67 & 22.7 & 0.7 & 55721.32 & 55735.46 \\
55763.68 & 22.6 & 0.2 & 55750.18 & 55775.31 \\
55788.93 & 21.0 & 0.2 & 55781.21 & 55794.51 \\
55999.68 & 21.8 & 0.4 & 55922.10 & 56041.48 \\
\hline
\end{tabular}

Table 3

PAIRITEL $J H K$-band Photometry of PTF 10nvg

\begin{tabular}{lcccccc}
\hline \hline Epoch (MJD) & $J$ mag & $J$ err & $H$ mag & $H$ err & $K$ mag & $K$ err \\
\hline 55387.376 & 12.229 & 0.025 & 10.232 & 0.030 & $8.456^{\mathrm{s}}$ & $0.060^{\mathrm{s}}$ \\
55392.294 & 12.231 & 0.026 & 10.231 & 0.030 & $8.475^{\mathrm{s}}$ & $0.063^{\mathrm{s}}$ \\
55450.191 & 10.896 & 0.025 & $9.414^{\mathrm{s}}$ & $0.031^{\mathrm{s}}$ & $8.029^{\mathrm{s}}$ & $0.060^{\mathrm{s}}$ \\
55463.151 & 11.538 & 0.025 & 9.801 & 0.030 & $8.303^{\mathrm{s}}$ & $0.060^{\mathrm{s}}$ \\
55466.126 & 11.585 & 0.025 & 9.966 & 0.030 & $8.347^{\mathrm{s}}$ & $0.060^{\mathrm{s}}$ \\
$\ldots$ & $\ldots$ & $\ldots$ & $\ldots$ & $\ldots$ & $\ldots$ & $\ldots$ \\
$\ldots$ & $\ldots$ & $\ldots$ & $\ldots$ & $\ldots$ & $\ldots$ & $\ldots$ \\
$\ldots$ & $\ldots$ & $\ldots$ & $\ldots$ & $\ldots$ & $\ldots$ & $\ldots$ \\
$\ldots$ & $\ldots$ & $\ldots$ & $\ldots$ & $\ldots$ & $\ldots$ & $\ldots$ \\
$\ldots$ & $\ldots$ & $\ldots$ & $\ldots$ & $\ldots$ & $\ldots$ & $\ldots$ \\
$\ldots$ & $\ldots$ & $\ldots$ & $\ldots$ & $\ldots$ & $\ldots$ & $\ldots$ \\
$\ldots$ & $\ldots$ & $\ldots$ & $\ldots$ & $\ldots$ & $\ldots$ & $\ldots$ \\
\hline
\end{tabular}

Notes.

s Photometry measured on short read PAIRITEL images (see the text).

n No reliable photometric measurements are available due to large thermal backgrounds (see the text).

(This table is available in its entirety in machine-readable and Virtual Observatory (VO) forms in the online journal. A portion is shown here for guidance regarding its form and content.)

et al. 2009). The "short-frame" mosaics contain a few dozen bright 2MASS stars which serve as calibration sources.

PAIRITEL has a known systematic uncertainty of a few percent on all flux measurements in each of the $J, H$, and $K_{s}$ bands (see Blake et al. 2008; Perley et al. 2010), which is larger than the typical statistical uncertainties. Following the method in Perley et al. we estimate the systematic uncertainties by measuring the scatter in the calibrated flux measurements of several (11 in this case) bright stars, arriving at 0.025 , 0.03 , and $0.06 \mathrm{mag}$ in the $J, H$, and $K_{s}$ bands, respectively. These systematic uncertainties are added in quadrature with the statistical uncertainties to provide the final uncertainties on each measurement. We note that occasionally large thermal backgrounds prevented the detection of PTF 10nvg in the $K_{s}$-band images, which has affected some of the epochs included in our analysis and results in only $J$ - and $H$-band measurements being reported.

The near-infrared light curve of PTF 10nvg is shown in Figure 1 and the photometric measurements are summarized in Table 3, which marks measurements made on short-frame mosaics as well as those affected by thermal background.

\subsubsection{Mid-infrared Data from WISE}

The Wide-field Infrared Survey Explorer (WISE) observed PTF 10nvg as part of routine survey operations, obtaining several tens of scans in late May and early June of 2010. This was around the time of the first peak but before the absolute peak brightness in the optical light curve shown in
Figure 1. The reported WISE photometry is partially saturated in bands $W 1(3.35 \mu \mathrm{m}), W 2(4.60 \mu \mathrm{m})$, and $W 3(11.56 \mu \mathrm{m})$ with $12 \%-15 \%$ of the pixels in the PSF in the saturated regime. The profile-fitted magnitudes account for the saturation while the aperture magnitudes are lower limits to the fluxes. The $W 4(22.24 \mu \mathrm{m})$ band photometry is not affected by saturation. Additional mid-infrared observations with WISE, ${ }^{9}$ as well as Spitzer and Herschel, ${ }^{10}$ have also been obtained.

\subsubsection{Millimeter-wave Data from CARMA}

PTF 10nvg was observed in the $2.7 \mathrm{~mm}$ continuum and several molecular lines with the Combined Array for Research in Millimeter-wave Astronomy (CARMA), located at an altitude of 7200 feet in the Inyo mountains of eastern California. The observations used six $10.4 \mathrm{~m}$ diameter antennas and nine $6.1 \mathrm{~m}$ diameter antennas. C-configuration, which provides baseline lengths between 26 and $370 \mathrm{~m}$, data were acquired on UT 2010 November 1 and 2, near the 2010 photometric minimum. Additional observations in the D-configuration, with baseline lengths between 11 and 148 m, were obtained on UT 2012 June 22 and 23.

For the C-configuration observations, the local oscillator was set to a frequency of $107.7 \mathrm{GHz}$. The CARMA correlator contains eight bands. Six bands were configured to have $487 \mathrm{MHz}$ bandwidth to observe the continuum. The remaining two bands were set to observe the $J=1-0$ rotational transitions of ${ }^{12} \mathrm{CO}(115.271204 \mathrm{GHz})$ and ${ }^{13} \mathrm{CO}(110.201353 \mathrm{GHz})$ with $0.98 \mathrm{kHz}$ resolution $\left(\sim 0.25 \mathrm{~km} \mathrm{~s}^{-1}\right)$. Time-variable atmospheric and instrumental gains were calibrated by observing the source J2038+513 every 20 minutes. Between gain calibrator observations, PTF 10nvg was observed for 7 minutes, followed by a 7 minute observation of a second embedded star in the North America Nebula. The passband amplitude and phases were calibrated using observations of $3 \mathrm{C} 454.3$ on each night. The absolute flux was calibrated by observing Uranus on November 1 and Neptune on November 2; the estimated absolute flux uncertainty is $10 \%$. The visibility data were calibrated using the MIRIAD reduction package.

The D-configuration observations were similar to the C-configuration observations, with the following differences. Two of the correlator bands were centered on $\mathrm{CN}, N=$ $1-0 \quad J=3 / 2-1 / 2(113.490982 \mathrm{GHz})$, and $\mathrm{C}^{18} \mathrm{O}, J=1-0$ $(109.78216 \mathrm{GHz})$, but these data are not presented here. The passband was calibrated with observations of 3C84. Flux calibration was performed by observing MWC 349 on the first night

\footnotetext{
9 The post-cryogenic phase of the WISE mission covered the field again in late November of 2010 . These would consist of only $W 1$ and $W 2$ data, but as the observations took place near the source's photometric minimum for the 2010 season they could be unsaturated. These data are not yet available in the WISE archive.

10 Spitzer has observed this source at several epochs during 2011 and 2012 (program PI: Abraham). Herschel has also observed PTF 10nvg (program PI: A. Kóspál) obtaining imaging photometry at 70,100, and $160 \mu \mathrm{m}$ at several epochs as well as single epoch 250,350 , and $500 \mu \mathrm{m}$ data.
} 

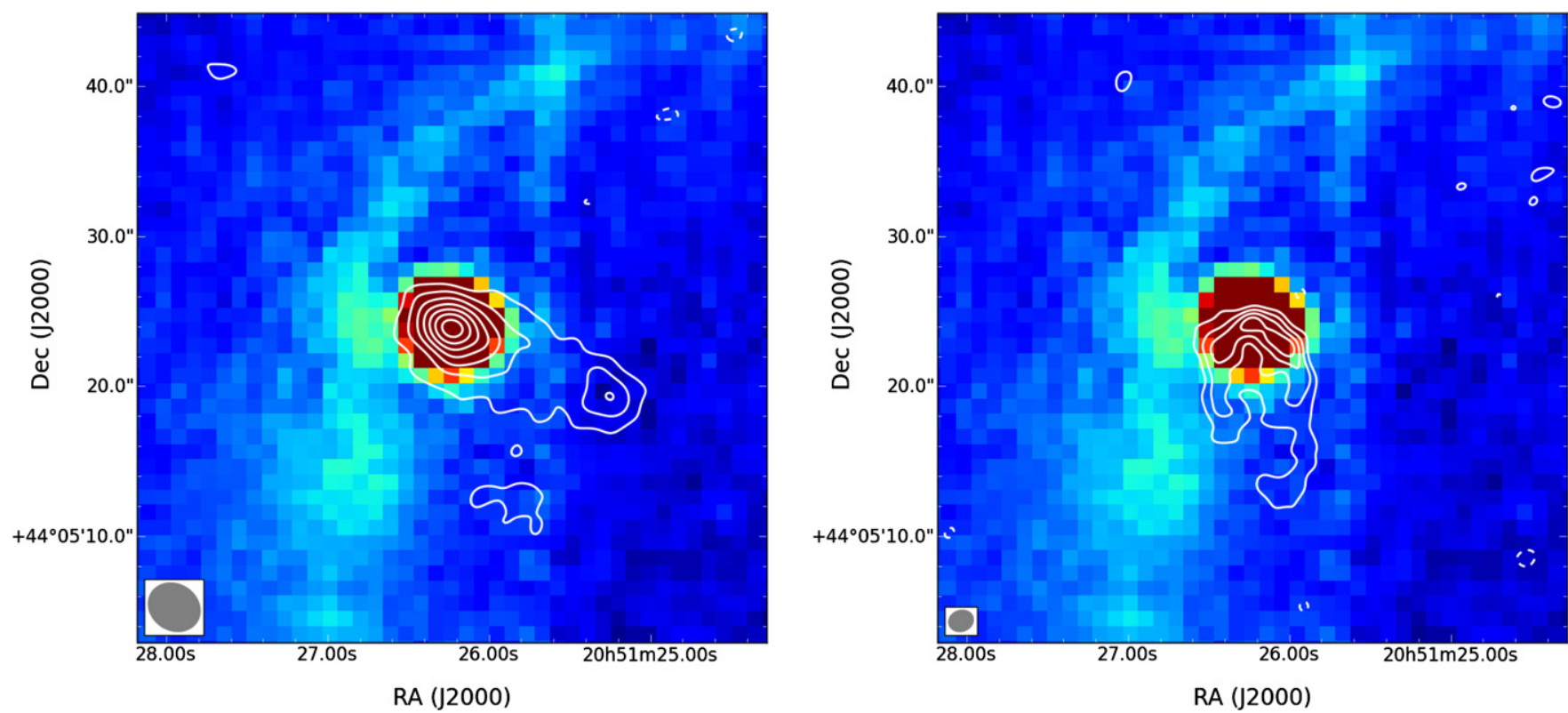

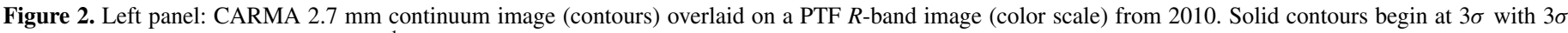

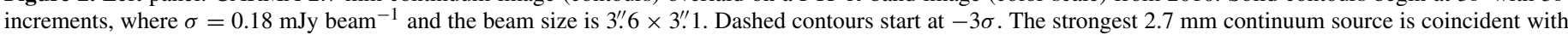

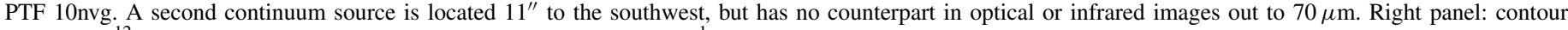

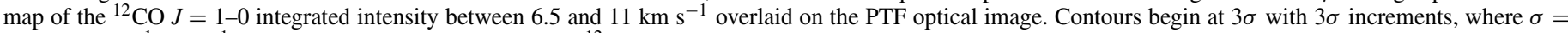

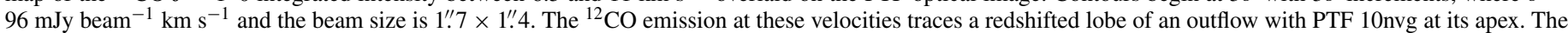
corresponding blueshifted lobe of the outflow was not detected.

(A color version of this figure is available in the online journal.)

and Neptune on the second night. Finally, on the second night in D-configuration, a seven-point mosaic was obtained to image the larger scale molecular cloud.

Continuum images were generated by inverting the complex visibilities using "natural" weighting for optimal pointsource sensitivity. The images were then deconvolved using the MIRIAD task mossdi. The rms noise in the image measured in emission-free regions near the vicinity of PTF $10 \mathrm{nvg}$ is $0.18 \mathrm{mJy}^{\text {beam }}{ }^{-1}$, with an FWHM beam size of 3 ". $6 \times 3$ ". 1 . Figure 2 shows the $2.7 \mathrm{~mm}$ continuum map overlaid on a PTF optical image. A compact source with a signal-to-noise ratio of 21 is detected in the field at a right ascension and declination of 20:51:26.2, $+44: 05: 23.8 \mathrm{~J} 2000$, corresponding to the optical/near-infrared position of PTF 10nvg. A secondary compact peak in the continuum image is located $11^{\prime \prime}$ to the southwest; this peak has no counterpart in the optical image shown, or in archival ground-based or Spitzer images between 1.2 and $70 \mu \mathrm{m}$.

The integrated $2.7 \mathrm{~mm}$ continuum flux toward PTF 10nvg is $5.60 \pm 0.26 \mathrm{mJy}$ (random error), obtained by fitting an elliptical Gaussian to the image. Formally the source is extended relative to the synthesized beam with a deconvolved size of $2^{\prime \prime} .7 \times 1^{\prime \prime} .5$ and a position angle of $66^{\circ}$. However, when imaged with the C-configuration data only, the source is unresolved with a synthesized beam of $1^{\prime \prime} .8 \times 1^{\prime \prime} .5$ and an integrated flux of $3.2 \pm 0.30 \mathrm{mJy}$. Similar combinations of extended and point source components have been observed in protostellar sources (e.g., Jørgensen et al. 2004), and have been interpreted to represent a resolved envelope and a compact disk. The presence of these structures in PTF 10nvg are consistent with the Class I classification from the shape of the SED.

The ${ }^{12} \mathrm{CO}$ and ${ }^{13} \mathrm{CO}$ spectral line images were generated using a Brigg's robust factor of zero to yield a beam size of $3^{\prime \prime} .2 \times 2$ ". 8 .
Since the emission is extended over several arcminutes, we used the maximum entropy program in MIRIAD (mosmem) to deconvolve the images. However, we caution that since no zero spacing data are available, the extended features are not reliably recovered in these images. Figure 3 shows images of the integrated ${ }^{12} \mathrm{CO}$ and ${ }^{13} \mathrm{CO}$ intensity between velocities of 3 and $6.25 \mathrm{~km} \mathrm{~s}^{-1}$, which covers the main part of the cloud.

PTF 10nvg, denoted by the cross $(x)$ in the figure, is coincident with a local maximum in the ${ }^{12} \mathrm{CO}$ and ${ }^{13} \mathrm{CO}$ emission. Emission from the larger scale extends to the west and southwest over a spatial extent of at least $0.18 \mathrm{pc}$. The full extent of the local cloud has not been encompassed with the CARMA mosaic, however. The detailed spatial structure of the extended cloud differs in the ${ }^{12} \mathrm{CO}$ and ${ }^{13} \mathrm{CO}$ images, which we attribute both to differences in optical depth and the lack of sensitivity to extended emission with an interferometer.

To the east, both the ${ }^{12} \mathrm{CO}$ and ${ }^{13} \mathrm{CO}$ emissions fall off sharply, suggesting that this structural feature has been robustly imaged with CARMA; PTF 10nvg is located along this ridge. The white contours in Figure 3 show the PTF optical image, which encompasses the $\mathrm{H} \alpha$ line. The $\mathrm{H} \alpha$ emission peaks to the east of the ${ }^{12} \mathrm{CO}$ and ${ }^{13} \mathrm{CO}$ emission, and closely follows the edge of the molecular cloud. Thus the eastern edge of the cloud appears to be illuminated by stars that are photodissociating the $\mathrm{H}_{2}$ on the cloud surface.

Figure 4 shows the ${ }^{12} \mathrm{CO}$ and ${ }^{13} \mathrm{CO}$ spectra within $\pm 2^{\prime \prime}$ of PTF 10nvg. Both ${ }^{12} \mathrm{CO}$ and ${ }^{13} \mathrm{CO}$ contain strong emission at a velocity of $\sim 5 \mathrm{kms}^{-1}$, which corresponds to the systematic velocity of the cloud. As shown in Figure 3, the molecular emission at these velocities contains a local maximum at the position of PTF 10nvg. The ${ }^{12} \mathrm{CO}$ line profiles in Figure 4 also display weaker emission that is redshifted up to $10-15 \mathrm{~km} \mathrm{~s}^{-1}$ from the systemic cloud velocity. The right panel in Figure 2 


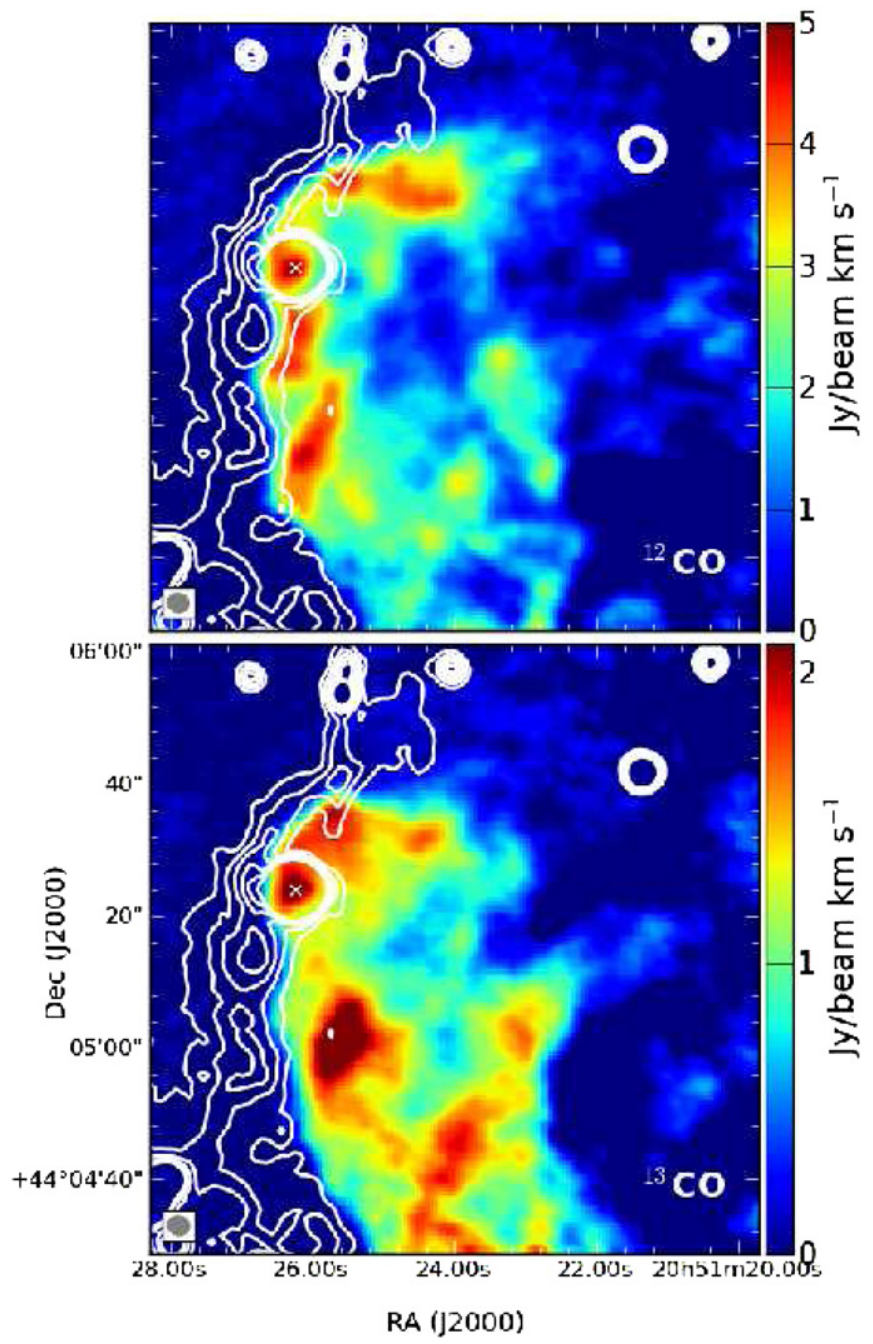

Figure 3. Wide-field view showing velocity-integrated $\left(3-6.25 \mathrm{~km} \mathrm{~s}^{-1}\right){ }^{12} \mathrm{CO}$ (top panel) and ${ }^{13} \mathrm{CO}$ (bottom panel) images in the color scale compared with PTF $R$-band intensity in the contours. Note the reversal in color vs. contour representation compared to Figure 2. The location of PTF 10nvg is marked by the " $x$ " toward the upper left. A remarkable alignment is found between the arc of $\mathrm{H} \alpha$ nebulosity (see also Figure 5) and the eastern edge of the $\mathrm{CO}$ emission.

(A color version of this figure is available in the online journal.)

shows an image of the redshifted image constructed from the $\mathrm{C}$-configuration data. The ${ }^{12} \mathrm{CO}$ emission at these velocities traces an arc-like structure extending to the south, with PTF 10nvg at the apex. The morphology and the redshifted velocities indicate an outflow from the young (proto)star. No evidence is seen in the ${ }^{12} \mathrm{CO}$ spectra for a corresponding blueshifted component of an extended flow.

\subsection{Adaptive Optics Imaging}

To assess the multiplicity of PTF 10nvg at wide orbital separations, we acquired high spatial resolution near-infrared images on UT 2010 September 25 using NIRC2 (K. Matthews) and the Keck II adaptive optics system at Mauna Kea. The source was sufficiently bright during its outburst phase to serve as its own natural guide star at the time of our observations. We obtained 18 unocculted, dithered frames in the $K_{s}$ band totaling $135 \mathrm{~s}$ of on-source integration time. The NIRC2 narrow camera mode provided fine spatial sampling (10 mas pixel ${ }^{-1}$ ) of the stellar PSF (FWHM $\approx 65$ mas for these observations).
Raw frames were processed by cleaning hot pixels, subtracting background noise, and aligning and co-adding the results. As discussed in Section 6, no companions are found in these observations.

\subsection{New Spectroscopy}

Tables 4 and 5 describe the optical and infrared spectroscopy obtained by our collaboration of PTF 10nvg since its photometric outburst in 2010. Low-resolution optical spectroscopy generally covering the red optical spectral region (>6200 $)$ were acquired at over 20 epochs with the facility spectrographs at one of the Keck I 10 m (LRIS; Oke et al. 1995; McCarthy et al. 1998; Steidel et al. 2004), Palomar 5 m (DBSP; Oke \& Gunn 1982), or Lick 3 m (Kast; Miller \& Stone 1993) telescopes. High-dispersion optical data were also obtained at Keck I using HIRES (Vogt et al. 1994) on six occasions. In the infrared, low-resolution data covering approximately $1-2.5 \mu \mathrm{m}$ were obtained at over 10 epochs using facility spectrographs at the $3 \mathrm{~m}$ Infrared Telescope Facility (SPeX; Rayner et al. 2003), Palomar Observatory $5 \mathrm{~m}$ (TripleSpec; Herter et al. 2008), and $3.5 \mathrm{~m}$ Apache Point Observatory (TripleSpec; Wilson et al. 2004). High-dispersion infrared data in the $Y$ band ( $1 \mu \mathrm{m}$ atmospheric window) were also obtained, at Keck II using NIRSPEC (McLean et al. 1998) at three epochs.

The data acquisition strategy included sensitivity to obtaining observations at the parallactic angle, and to proper acquisition of flux calibration standards. Spectral data processing followed standard procedures for bias level correction, flat fielding, spectral extraction including background subtraction, and flux calibration for the low-dispersion data. Of note is the extended emission from a nebular arc region about 7 " away from PTF 10nvg (see Figure 3 or 5 for morphology) that must be accounted for when performing background subtraction on spectra. The absolute calibration of the low-dispersion data is typically good to $\pm 20 \%$ and slightly better under photometric conditions. The resulting signal-to-noise ratios in the optical spectra range from 15 to 45, but lower in the 2011 late June to early August time frame when the source was optically very faint. For the moderate resolution infrared spectra, the signal-to-noise ratio was typically $\sim 250$ in the $K$ band and $\sim 40$ in the $J$ band though varied by factors of a few depending on the weather and source brightness, and color at any particular epoch.

\section{PHOTOMETRIC ANALYSIS}

\subsection{Optical and Near-infrared Variability}

The light curves published in Covey et al. included data obtained through 2010 December 14 (JD 2455545). Updated multi-wavelength light curves appear in Figure 1 and show that the source continued its large-amplitude fluctuations throughout the 2011 and 2012 observing seasons. The data do not completely sample the photometric behavior, notably during the period when the field was too close to the Sun from approximately late December to early February of each year or during survey-designed gaps in the $R_{\mathrm{PTF}}$ observing, as well as when the source was optically faint and below the PTF detection limits. Our near-infrared observations, in addition to the gaps in coverage associated with solar conjunction, have large annular gaps in August when Mt. Hopkins is closed due to the Arizona monsoon season. Despite the incomplete sampling, the photometric evolution of PTF 10nvg is obviously dominated by large-amplitude variations on several month timescales, with superposed chaotic behavior on shorter timescales. 
Table 4

Summary of Optical Spectra

\begin{tabular}{|c|c|c|c|c|c|}
\hline UT Date & $\begin{array}{l}\text { Wavelength } \\
\text { Range ( }(\AA)\end{array}$ & $\begin{array}{l}\text { Spectral } \\
\text { Resolution }\end{array}$ & $\begin{array}{l}\text { Telescope/ } \\
\text { Instrument }\end{array}$ & $\begin{array}{l}\text { Slit } \\
\text { P.A. }\end{array}$ & Observers \\
\hline $2010 \mathrm{Jul} 8$ & $3010-10200$ & $4 / 7 \AA$ & Keck/LRIS-Blue+Red & 113.0 & Bloom, Cucchiara, Morgan, Perley \\
\hline $2010 \mathrm{Jul} 19$ & $3452-10800$ & $4 / 10 \AA$ & Lick/Kast-Blue+Red & 117.6 & Griffith, Kandrashoff \\
\hline 2010 Aug 12 & $3400-5500 / 6300-8800$ & $2 / 5 \AA$ & Palomar $200^{\prime \prime} /$ DoubleSpec-Blue + Red & 268.0 & Rebull \\
\hline 2010 Sep 16 & $3500-10000$ & $4 / 10 \AA ̊$ & Lick/Kast-Blue+Red & 186.4 & Cenko \\
\hline 2010 Dec 5 & $6440-7105$ & $2.6 \AA$ & Palomar $200^{\prime \prime}$ / DoubleSpec-Red & 97.0 & Hillenbrand, Mooley \\
\hline 2010 Dec 13 & $4310-8770$ & HIRES & Keck/HIRES & 110.8 & Hillenbrand, Kraus, Law \\
\hline 2011 May 3 & $3440-10700$ & $4 / 10 \AA$ & Lick/Kast-Blue+Red & 90.8 & Barth \\
\hline 2011 Jun 3 & $3370-10200$ & $4 / 10 \AA$ & Keck/LRIS-Blue+Red & 166.0 & Silverman, Filippenko, Cenko \\
\hline 2011 Jun 15 & $3645-7975$ & HIRES & Keck/HIRES & 176.0 & Hillenbrand \\
\hline 2011 Jun 28 & $4315-8775$ & HIRES & Keck/HIRES & 16.9 & Kraus, Law \\
\hline 2011 Jun 29 & $3324-10150$ & $4 / 10 \AA$ & Keck/LRIS-Blue+Red & 124.0 & Silverman, Cenko, Nugent \\
\hline $2011 \mathrm{Jul} 4$ & $6165-6832$ & $2.6 \AA$ & Palomar $200^{\prime \prime} /$ DoubleSpec-Red & 198.0 & Kirby \\
\hline $2011 \mathrm{Jul} 5$ & $3500-9816$ & $4 / 10 \AA$ & Lick/Kast-Blue+Red & 132.8 & Graham, Hsiao \\
\hline 2011 Aug 1 & $3298-10200$ & $4 / 10 \AA$ & Keck/LRIS-Blue+Red & 125.0 & Silverman, Cenko \\
\hline 2011 Aug 2 & $3276-10200$ & $4 / 10 \AA$ & Keck/LRIS-Blue+Red & 125.0 & Silverman, Cenko \\
\hline 2011 Aug 28 & $3184-10200$ & $4 / 10 \AA$ & Keck/LRIS-Blue+Red & 107.0 & Silverman, Cenko, Miller \\
\hline 2011 Aug 31 & $3300-5200 / 6100-8250$ & $8 / 10 \AA$ & Palomar $200^{\prime \prime} /$ DoubleSpec-Blue + Red & 210.0 & Liu \\
\hline 2011 Oct 25 & $3432-10246$ & $4 / 10 \AA$ & Lick/Kast & 133.6 & Kandrashoff, Blanchard, Silverman \\
\hline 2011 Oct 30 & $3500-10185$ & $4 / 10 \AA$ & Palomar 200" /DoubleSpec-Blue + Red & unk. & Tendulkar, Ofer \\
\hline 2011 Nov 26 & $4350-10260$ & $4 / 6 \AA$ & Keck/LRIS-Blue+Red & 93.6 & Silverman, Clubb \\
\hline 2011 Dec 9 & $4775-9220$ & HIRES & Keck/HIRES & 102.6 & Hillenbrand \\
\hline 2012 Jan 1 & $4800-11000 \AA$ & $6 \AA$ & Palomar $200^{\prime \prime}$ / DoubleSpec-Red & 82.0 & Hillenbrand \\
\hline 2012 Jan 2 & $4800-11000 \AA$ & $6 \AA$ & Palomar $200^{\prime \prime}$ / DoubleSpec-Red & 81. & Hillenbrand \\
\hline 2012 Jan 6 & $4420-8770$ & HIRES & Keck/HIRES & 86.6 & Kraus, Law, Hillenbrand \\
\hline 2012 May 17 & $3230-10200$ & $4 / 6 \AA$ & Keck/LRIS-Blue+Red & 220.0 & Clubb, Silverman, Cenko, Filippenko, Miller \\
\hline 2012 Jul 11 & $3460-10395$ & $4 / 10 \AA$ & Lick/Kast-Blue+Red & 133 & Clubb, Silverman \\
\hline $2012 \mathrm{Jul} 28$ & $4000-6000 / 7750-9250$ & $4 / 10 \AA$ & Palomar 200" / DoubleSpec-Blue & 263.0 & Hillenbrand, David \\
\hline 2012 Aug 7 & $3640-7985$ & HIRES & Keck/HIRES & 91.3 & Marcy, Isaacson \\
\hline
\end{tabular}

Notes. Divider separates spectra published in Covey et al. (2011) from those newly presented here.

Table 5

Summary of Near-infrared Spectra

\begin{tabular}{lcccc}
\hline \hline UT Date & $\begin{array}{c}\text { Wavelength } \\
\text { Range }(\mu \mathrm{m})\end{array}$ & $\begin{array}{c}\text { Spectral } \\
\text { Resolution }\end{array}$ & $\begin{array}{c}\text { Telescope/ } \\
\text { Instrument }\end{array}$ & Observers \\
\hline 2010 Jul 14 & $0.8-2.5$ & $R \sim 2000$ & IRTF/SpeX & Rayner \\
2010 Jul 14 & $3-4.2,4.5-5.0$ & $R \sim 2500$ & IRTF/SpeX & Rayner \\
2010 Jul 16 & $0.95-1.12$ & $R \sim 25000$ & Keck/NIRSPEC & Hillenbrand \\
2010 Jul 18 & $1-2.5$ & $R \sim 5000$ & APO/Triplespec & Covey \\
\hline 2010 Sep 23 & $1-2.5$ & $R \sim 2700$ & Palomar/TripleSpec & Covey \\
2010 Nov 26 & $1-2.5$ & $R \sim 2700$ & Palomar/TripleSpec & Liu, Angione \\
2010 Dec 14 & $1-2.5$ & $R \sim 2700$ & Palomar/TripleSpec & Muirhead \\
2010 Dec 15 & $1-2.5$ & $R \sim 2700$ & Palomar/TripleSpec & Muirhead \\
2011 Jun 26 & $0.8-2.5$ & $R \sim 1200$ & IRTF/SpeX & Covey \\
2011 Jul 15 & $0.8-2.5$ & $R \sim 1200$ & IRTF/SpeX & Covey \\
2011 Aug 17 & $1-2.5$ & $R \sim 2700$ & Palomar/TripleSpec & Muirhead \\
2011 Sep 2 & $0.8-2.5$ & $R \sim 1200$ & IRTF/SpeX & Covey \\
2011 Sep 15 & $0.95-1.12$ & $R \sim 25000$ & Keck/NIRSPEC & Hillenbrand \\
2011 Oct 18 & $0.8-2.5$ & $R \sim 2000$ & IRTF/SpeX & Covey \\
2012 May 5 & $0.95-1.12$ & $R \sim 25000$ & Keck/NIRSPEC & Herczeg, Gong \\
\end{tabular}

Notes. Divider separates spectra published in Covey et al. (2011) from those newly presented here.

As described in Covey et al., PTF 10nvg was not a recognized optical or near-infrared source prior to its 2010 identification as an outbursting (possibly transient) source. It is noted in Aspin (2011), however, that the source is reported at faint optical magnitudes in the USNO-B1 catalog in 1979 November ${ }^{11}$ as

11 We were unable to find a POSS image of the field taken in 1979. However, in the digitized scans of all POSS images including the position of PTF 10nvg, the source is detected in one red plate taken in 1990 (see Figure 5). The $B$ and
$R$ mag measurements reported in Aspin (2011) correspond to the USNO-B1.0 source 1340-0394684, which has a cataloged position that is $\sim 3$ ". 4 away from the optically measured position of PTF $10 \mathrm{nvg}$. The USNO-B1.0 catalog reports an epoch of 1966.2 for this source, which is the result of considering the B1 and R1 plates from the early 1950s (on which the reported photometry is in fact likely related to the nebulosity rather than the point source), and the R2 plate from 1990 (on which there is a true source detection), with the final position given as the geometric mean of these three epochs after taking a potential proper motion term into account (S. Levine 2012, private communication). 


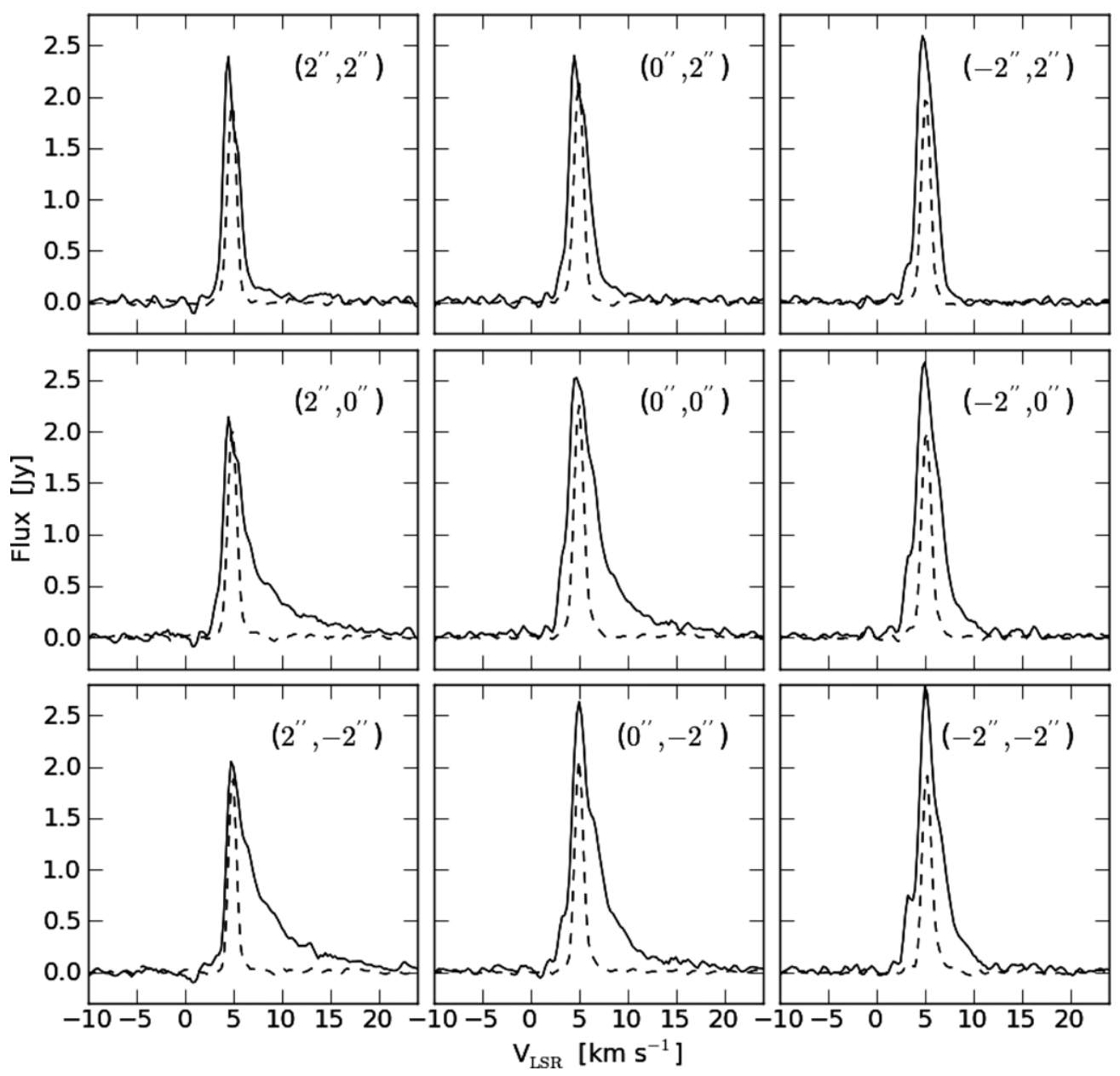

Figure 4. ${ }^{12} \mathrm{CO}$ (solid) and ${ }^{13} \mathrm{CO}$ (dashed) $J=1-0$ spectra in the vicinity of PTF 10nvg. Panels are labeled with positional offsets in arcseconds relative to the position of PTF 10nvg. Both the ${ }^{12} \mathrm{CO}$ and ${ }^{13} \mathrm{CO}$ data cubes contain strong emission at the cloud systemic velocity that peaks at the location of PTF $10 \mathrm{nvg}$ (see Figure 3 ). The ${ }^{12} \mathrm{CO}$ emission also displays redshifted emission that extends to the south of PTF 10nvg (see the right panel of Figure 2), representing a redshifted outflow.

well as in a 1997 October $30 \mathrm{H} \alpha$ image (their Figure 2); it is also recorded in UKIDSS infrared data from 2006 June. These detections led us to investigate the historical variability of PTF 10nvg via examination of images taken over several decades prior to the 2009 optical detection by PTF. In Figure 5, we show the vicinity of PTF 10nvg from several sources including the digitized plate scans of POSS-I and POSS-II, our own previously unpublished optical images from the KPNO-0.9 m, and those from SDSS. The apparitions of the source in 1990, 1997, and 2006 combined with the large number of non-detections in other imaging data sets spanning approximately 50 years (see Figure 5) hint that this young stellar object has displayed long-term, large-amplitude variability. Notably, although the 1997 October $\mathrm{H} \alpha$ detection of the point source exhibited by Aspin (2011) is convincing, the source was not detected in $B V R I$ or $\mathrm{H} \alpha$ images taken only a few months earlier (1997 August). Considering the body of evidence, the average faintstate magnitude in the red optical is likely below about $22 \mathrm{mag}$, based on the deepest digital data from the late 1990s. The source clearly has been intermittently detectable, however.

In 2009 August, the object was captured by PTF at around $R_{\mathrm{PTF}} \approx 17.2 \mathrm{mag}$ in what may have been the decline from an earlier local peak, with subsequent fading over a roughly 1 month timescale to $\approx 20$ mag. Our next observations were approximately 200 days later by which point PTF $10 \mathrm{nvg}$ had brightened by over 4 mag. It exhibited a first local peak in 2010
July, then dropped in brightness by more than a magnitude and rose to its maximum recorded brightness of $R_{\mathrm{PTF}} \approx 13.5 \mathrm{mag}$ approximately 40 days later, in 2010 August. The light curve published in Covey et al. demonstrate a third peak in early December of 2010.

During the 2011 and 2012 observing seasons, several subsequent peaks that were typically narrower in time and fainter than the initial two 2010 peaks have been observed, as well as a broad, bright peak exhibited in mid-2012. Notably, the declines from each peak were sharp, approximately $0.1 \mathrm{mag} \mathrm{day}^{-1}$, while the rise times were about half as steep. ${ }^{12}$ The total optical variability amplitude observed during the 2011 and 2012 seasons was more than $4.5 \mathrm{mag}$, with PTF 10nvg as bright as $R_{\mathrm{PTF}} \sim 15 \mathrm{mag}$ in early 2011 and repeatedly reaching $R_{\mathrm{PTF}} \sim 16$ mag during the rest of 2011 and 2012. The source was also repeatedly fainter than the P48 $60 \mathrm{~s}$ detection limit $(\approx 20.5 \mathrm{mag}$ in this high background field). Stacked images from these prolonged faint periods (e.g., between late June through late August in 2011 and again from late December 2011 through mid-March 2012) indicate that the average flux from PTF 10nvg reached as faint as $R_{\mathrm{PTF}} \sim 23 \mathrm{mag}$ (see Section 2.1.1). Near-infrared

\footnotetext{
12 The individually measured slopes for events lasting longer than a week are $0.14,0.14$, and 0.14 mag day $^{-1}$ for the drops following the peaks that occurred on JD 2455678, 2455884, and 2456086, respectively. For the rise times, the measured slopes are $\sim 0.05,0.05$, and $0.07 \mathrm{mag} \mathrm{day}^{-1}$ characterizing the events starting on JD 2455652, 2455820, and 2456058, respectively.
} 


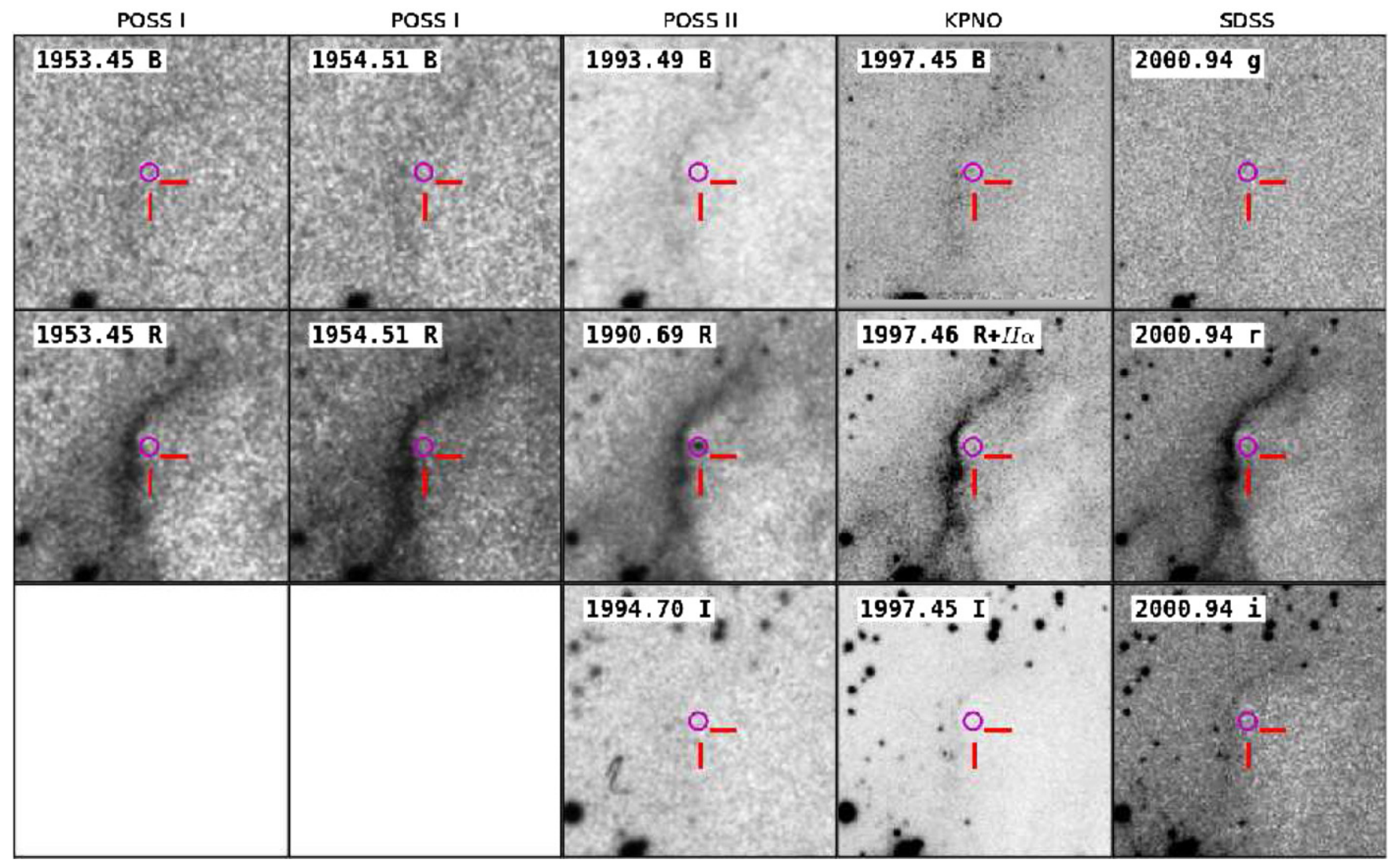

Figure 5. Optical images with north upward and east to the left covering $96^{\prime \prime}$ of the PTF 10nvg vicinity, spanning many decades. Rows contain blue, red, and far-red data from top to bottom, while the columns contain different imaging surveys, as indicated above the figure. The individual panels correspond to different epochs. PTF 10nvg is located at the center of the magenta circle and is not visible at most epochs, though was apparent on the $1990 R$-band plate. The intersection of the red cross hairs indicates the position of the USNO-B source identified in Aspin (2011) as the likely quiescent counterpart to PTF 10nvg but no source is detected at this position in any of the POSS I plate scans.

(A color version of this figure is available in the online journal.)

detections continued through the periods of optical dimming and at some epochs better sample the source fading and rising while it remained optically faint.

The infrared light curves (Figure 1) generally follow the fading and peaking behavior seen in the optical, with no detectable time lag between the optical and infrared valleys and peaks seen within our coarse sampling. However, the amplitudes are different between the $R J H K_{s}$ bands, and indicate large color variations. PAIRITEL obtains data simultaneously in the $J, H$, and $K_{s}$ bands allowing for precise measurements of color change in the near-infrared. A color-color diagram (left side of Figure 6) indicates that during its large-amplitude brightness changes, the color evolution of PTF 10nvg does not deviate strongly from the behavior expected from variations in line-ofsight extinction. The observed scatter around the expected relationship for time-variable obscuration by standard dust grains is roughly consistent with the typical observational uncertainties. A color-magnitude diagram (right side of Figure 6) reinforces the finding that the source becomes redder when fainter in a manner consistent with extinction. ${ }^{13}$

\footnotetext{
13 We note that the same conclusion is not reached in Aspin (2011) based on an analysis of the UKIDSS detection from 2006 and seven additional observations made between 2010 July and 2010 September. We find that indeed the 2006 detection by UKIDSS $\left(J-H=2.85 \mathrm{mag} ; H-K_{s}=3.4 \mathrm{mag}\right.$ ) lies far from the ensemble of 2010 outburst through 2012 points measured by PAIRITEL, as shown in Figure 6. However, the deviation argued for in Aspin (2011) of the observations taken during outburst from standard reddening laws appears to be the result of an assumption that the unreddened origin for PTF
}

Assuming a standard interstellar extinction law and that all of the observed near-infrared color variations are the result of changes in extinction along the line of sight (rather than to an outburst), we find that the observed excursions in nearinfrared color-color and color-magnitude diagrams correspond to variations in extinction of $\Delta A_{V} \gtrsim 30 \mathrm{mag}$ (see also the discussion in Section 5 related to Figure 9). Extending the analysis, if we adopt the position that all of the historical photometric variation observed for this source can be attributed to changes in line-of-sight extinction, the 2MASS non-detection of the source at the $K_{s}$ band relative to the peak brightness observed with PAIRITEL implies $\Delta A_{V} \approx 70 \mathrm{mag}$.

We sought next to quantify the timescales associated with the observed large-amplitude systematic changes in source brightness. Some general challenges for interpreting the light curve of PTF 10nvg include (1) the inhomogeneous sampling that fails to resolve all the peaks and valleys in the light curve; (2) the limited time baseline of the observations that may not properly resolve long-term trends; and (3) the likely possibility

10nvg lies along the "classical T Tauri locus." As shown in both Covey et al. and Aspin (2011), however, PTF 10nvg was not a classical T Tauri star prior to outburst, but rather a Class I-type source. As Class I sources have both envelope and disk emission contributing to the near-infrared colors, there is no reason to expect that the origin of the reddening vector should lie on the classical T Tauri star locus. This realization does not, however, explain the discrepant colors reported by UKIDSS. One possibility is that in its faint state the source becomes more dominated by scattered light which could explain the turn blueward in the $J-H$ color. However, the $H-K$ discrepancy seems to require redder colors at this epoch rather than bluer. 

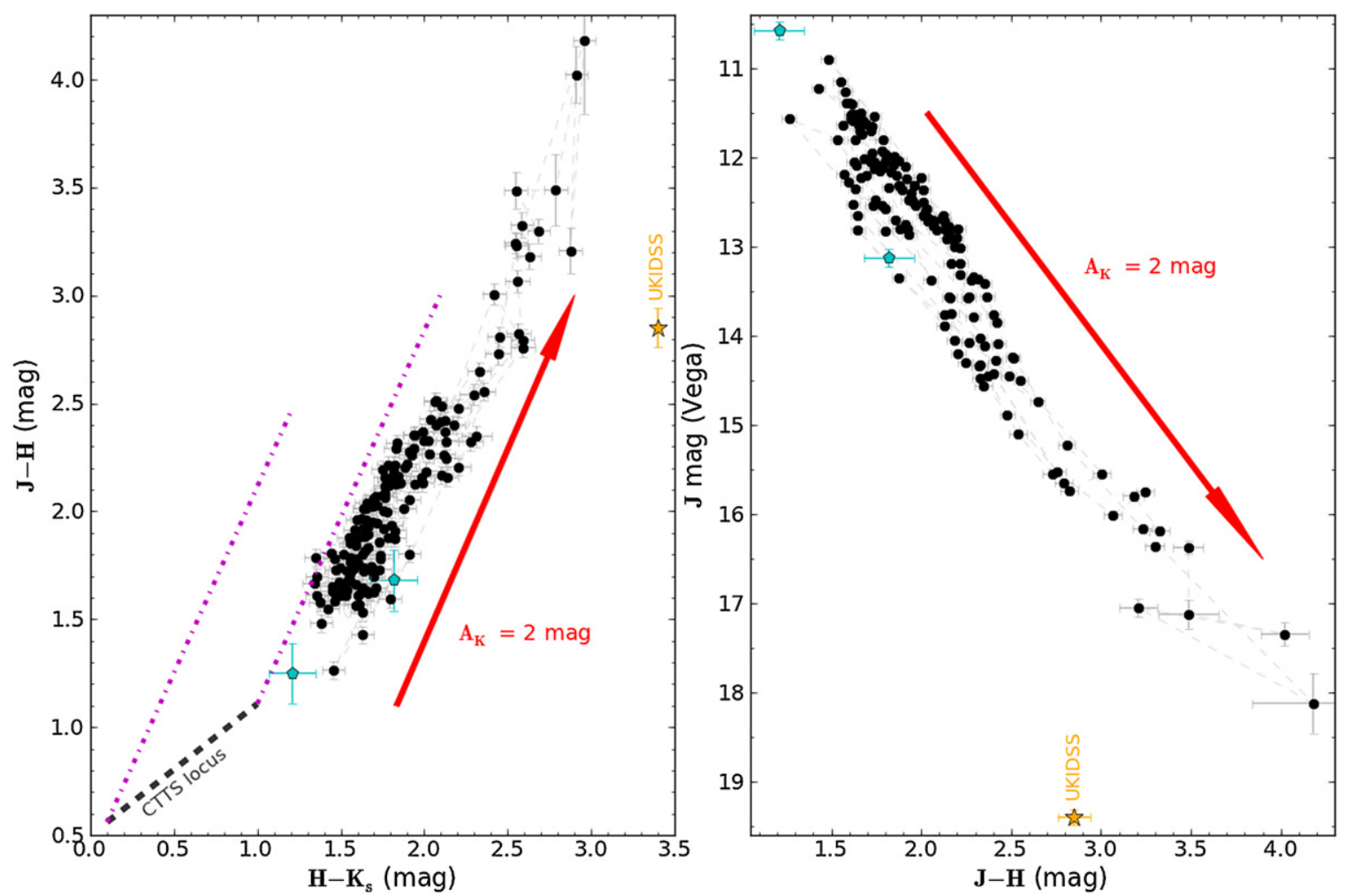

Figure 6. Color-color and color-magnitude diagrams with the $J H K_{s}$ light curve data exhibited in Figure 1 shown as black points. Cyan pentagons show two measurements from Aspin (2011) while the gold star shows the 2006 measurement from UKIDSS. For reference, the dashed line indicates the locus of classical T Tauri stars as measured by Meyer et al. (1997) using the color transform of Carpenter (2001). When the overall features of the data set are considered, the time variation of the black and cyan points is consistent with the effects of extinction following an interstellar extinction law from Indebetouw et al. (2005), plotted in dash-dotted lines. The point-to-point variations, however, may require additional thermal effects beyond the dominant extinction effect.

(A color version of this figure is available in the online journal.)

that the system is dynamic, meaning that periods, amplitudes, and relative phases could be changing cycle to cycle.

In our search for periodic signals in the brightness variations of PTF 10nvg we use a generalized Lomb-Scargle periodogram (Lomb 1976; Scargle 1982; Zechmeister \& Kürster 2009) and analyze each photometric band separately (see Richards et al. 2011 for more details on our implementation). The extended period of non-detections in the $R_{\text {PTF }}$ band would lead to a biased periodogram since the framework for the Lomb-Scargle analysis does not account for upper limits. As a result, we focus on the three near-infrared bands. The form of the long-term secular changes in the light curve is difficult to identify over the relatively limited timescales that we probe. Thus, we perform our Lomb-Scargle analysis by simultaneously fitting for the periodic and long-term linear trend in the data. The observations do not clearly justify the use of a higher order polynomial, yet we note that simultaneously fitting for either a quadratic or cubic long-term trend does not significantly alter the results described below.

In Figure 7, we show the periodogram for each of the three near-infrared bands, including observations taken prior to 2012 June 16 only. The periodogram exhibits significant power on long timescales. In each of the $J, H$, and $K_{s}$ bands we find a large peak in the periodogram corresponding to a period $P \sim 221$ days. The power at the 1 day parasite frequency, an artifact produced by the fact that we observe nightly, is strong, but weaker than the power inferred for the astrophysically significant period at 221 days. The peak is somewhat broad in frequency, perhaps indicating only semi-periodic behavior from cycle to cycle.

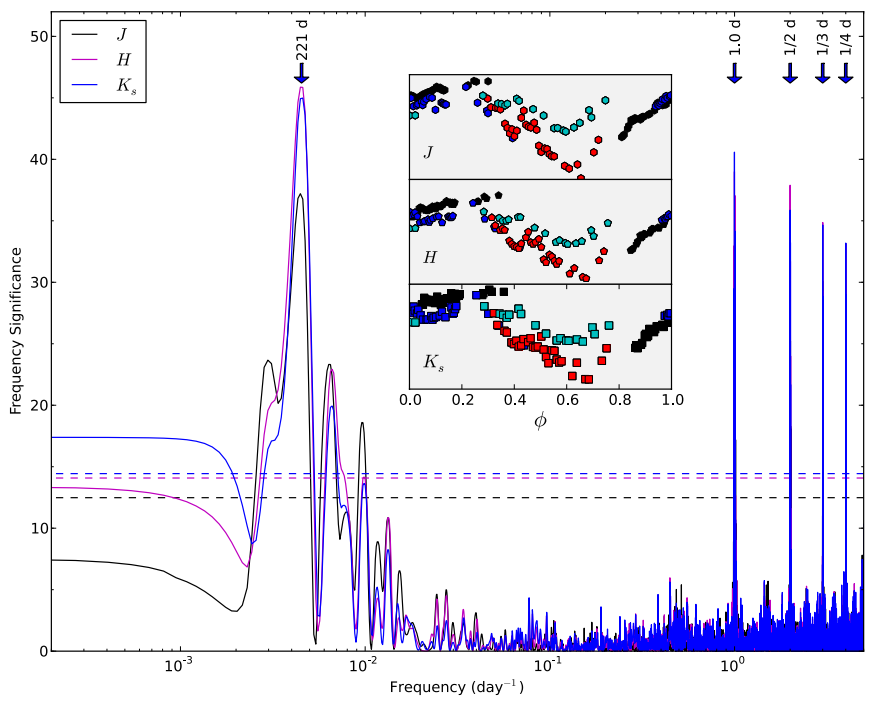

Figure 7. Analysis of timescales inferred from the photometric time series. Lomb-Scargle periodogram for each of the $J H K_{s}$ passbands. A period of around 221 days, though with a broad frequency peak, is apparent. It is well above the signal level of the calculated $0.1 \%$ false alarm probability signal, shown as the horizontal dashed lines and calculated using $10^{4}$ random re-shuffled instances of the data. Insets show the phased data, with the simultaneously fit linear trend removed for clarity of presentation. The colors indicate sequential observing seasons or other gaps in the data as follows: cyan, 2010; red, first part of 2011; blue, second part of 2011; black, 2012 prior to June.

(A color version of this figure is available in the online journal.) 
To quantify the significance of the observed peak in the periodogram we randomly resample the PAIRITEL flux measurements while fixing the cadence to match our observations. Following this we repeat the Lomb-Scargle analysis described above and measure the peak value in the resulting periodogram, while excluding the $1,1 / 2,1 / 3$, and $1 / 4$ days overtones of the 1 day alias. This procedure is repeated 10,000 times, which allows us to determine a "false alarm probability" that the observed peak in the periodogram is the result of noise by examining the rank ordered values of the peak significance from the 10,000 trials. In Figure 7, we show the $0.1 \%$ false alarm probability, meaning that a peak in the periodogram greater than this value has $<0.1 \%$ chance of being the result of random fluctuations. In each filter the peak is well above this value, which clearly shows that the measured periodicity is significant. This can visually be confirmed based on the phase-folded trend-removed light curves shown in the inset of Figure 7. We interpret these results as tentative evidence that the emission from PTF 10nvg varies periodically on timescales of roughly 221 days; however, we caution that the signal may be driven by the particular peaks and minima that we do observe, specifically the two deep minima observed near JD 2455520 and 2455760. With higher cadence observations taken over longer time baselines we will be able to confirm if the periodic signal observed in the 2010-2012 data set is fundamental to the system, or a peculiarity of the observing window through which we have measured the source.

Summarizing our findings, we see evidence that the largeamplitude $J H K_{s}$ near-infrared variations are semi-periodic, with a roughly 220 day timescale. Local peaks in the $R_{\mathrm{PTF}}$ optical light curve on JD $\approx 2455679,2455884$, and 2456084 reinforce this timescale. We note, however, that during the 2010 season in particular, the $R_{\text {PTF }}$ photometry does not phase well with the period derived from the near-infrared $\left(J H K_{s}\right)$ photometry. Further, beginning in the middle of 2012 June, the $R_{\text {PTF }}$ light curve clearly deviates from the previously observed semiperiodic behavior; rather than fading as expected based on the derived period, PTF 10nvg instead reached its brightest optical state since 2010. PAIRITEL sampling during this time frame is sparse due to the Arizona monsoon season, and corresponding telescope closure; nevertheless, the near-infrared observations confirm the deviation from the previously observed semiperiodic behavior. This suggests that while extinction clearly plays a prominent role in the variability, other processes such as variable accretion (which would affect optical photometry moreso than infrared photometry) could also be important in driving the photometric evolution of this source. As we discuss in Section 5, the correlation of enhanced optical $\mathrm{TiO} / \mathrm{VO}$ band emission (requiring heating and then cooling of molecular material) and permitted line emission (requiring accretion) with the bright state supports this explanation. Concerning the historical imaging of this source, while it was not visible to the limits of the 2MASS survey, not once over the past three years of our photometric monitoring has it been fainter than $K_{s} \approx 11 \mathrm{mag}$. Thus, although discussed above in the context of possible variations in extinction of $A_{V} \approx 70 \mathrm{mag}$, the recently bright $K_{s}$-band magnitude could instead support the importance of an outburst component, despite variable extinction clearly being part of the picture.

Should the periodic signal be confirmed, that in conjunction with the evidence that the photometric variations are largely due to changes in extinction along the line of sight (Figure 6) would naturally suggest that the recently observed long period variability arises in a dusty structure characterized by a dynamical time $\tau_{\text {dyn }} \sim 221$ day. If this is a rotating disk around a $0.5-1 M_{\odot}$ star, it would indicate higher opacity material in a clump or warp located around $0.5-0.7$ AU from the star. The implied orbital velocity for a circular orbit $\left(25 \mathrm{~km} \mathrm{~s}^{-1}\right)$ can be compared to the characteristic timescale for the photometric rises and declines that would correspond to repeated clearing and obscuration events. Referring to Figure 1, the relevant rise and fall times are a few tens of days. This timescale relative to the observed period is similar to the ratio between the derived location and the orbital circumference $(2 \pi)$, resulting in a size scale for the obscuring material that is thus similar to its distance from the star, namely $\sim 0.5 \mathrm{AU}$.

\subsection{Mid-infrared Variability}

The data acquisition strategy for the WISE mission included multiple scans of each point on the sky, which provides timeseries data on timescales that vary with ecliptic latitude. For the scans covering PTF 10nvg, the time-series data are rather concentrated, with several observations obtained within just a few several-day-long time frames. The observations took place around the time of the first peak and before the absolute peak brightness exhibited in the optical light curve (Figure 1). PTF 10 nvg is flagged in the WISE catalogs as a potential variable in the $W 3$ and $W 4$ bands based on 26 individual measurements. Examination of the photometry from these individual scans reveals nothing systematic about the behavior in time between, e.g., the 2010 May 27 and 28 WISE data versus the 2010 June 4 WISE data. Scatter among the many observations taken so close in time that is larger than the quoted errors likely leads to the variable source flag; it is not clear whether the source is actually varying on sub-day timescales, however.

Comparing the WISE photometry to previously reported measurements at similar wavelengths from Spitzer, MSX, and $I R A S$, illustrates that the source brightened in 2010 at midinfrared wavelengths as well as at the near-infrared and optical wavelengths highlighted in Covey et al. and discussed in detail above. Specifically, the Spitzer fluxes from 2006/2007 reported by Rebull et al. (2011) at both $3.6 \mu \mathrm{m}$ and $4.5 \mu \mathrm{m}$ were a factor of more than 10 lower than those recorded by WISE in 2010 at $3.35 \mu \mathrm{m}$ and $4.6 \mu \mathrm{m}$. The WISE $22 \mu \mathrm{m}$ measurement can be compared with data from not only Spitzer but also from earlier $\operatorname{MSX}(21.3 \mu \mathrm{m})$ and IRAS $(25 \mu \mathrm{m})$ measurements. While there are wavelength and beam size differences between these various missions to consider, it is clear that the 2010 WISE photometry is brighter by a factor of $\sim 4$ compared to the 2006 Spitzer $24 \mu \mathrm{m}$ data, with the earlier missions all reporting photometry at intermediate flux levels.

\section{SPECTRAL ENERGY DISTRIBUTION AND VARIABILITY}

In Figure 8, the outburst SED presented by Covey et al. is updated to include the photometry from WISE and CARMA.

Using the CARMA continuum flux measurement of $5.6 \mathrm{mJy}$ at $2.7 \mathrm{~mm}$, a total mass (dust + gas assuming a ratio of $1: 100$ ) of $0.06 M_{\odot}$ is calculated. This assumes a distance of $520 \mathrm{pc}$, a dust opacity of $0.01 \mathrm{~cm}^{2} \mathrm{~g}^{-1}$ at $107 \mathrm{GHz}$ (Beckwith et al. 1990), that the continuum emission is optically thin and isothermal with a temperature of $20 \mathrm{~K}$, and that free-free emission is a negligible contribution. This value is on the high end of circumstellar masses measured for young stars and consistent with the Class I-type SED of the source. 


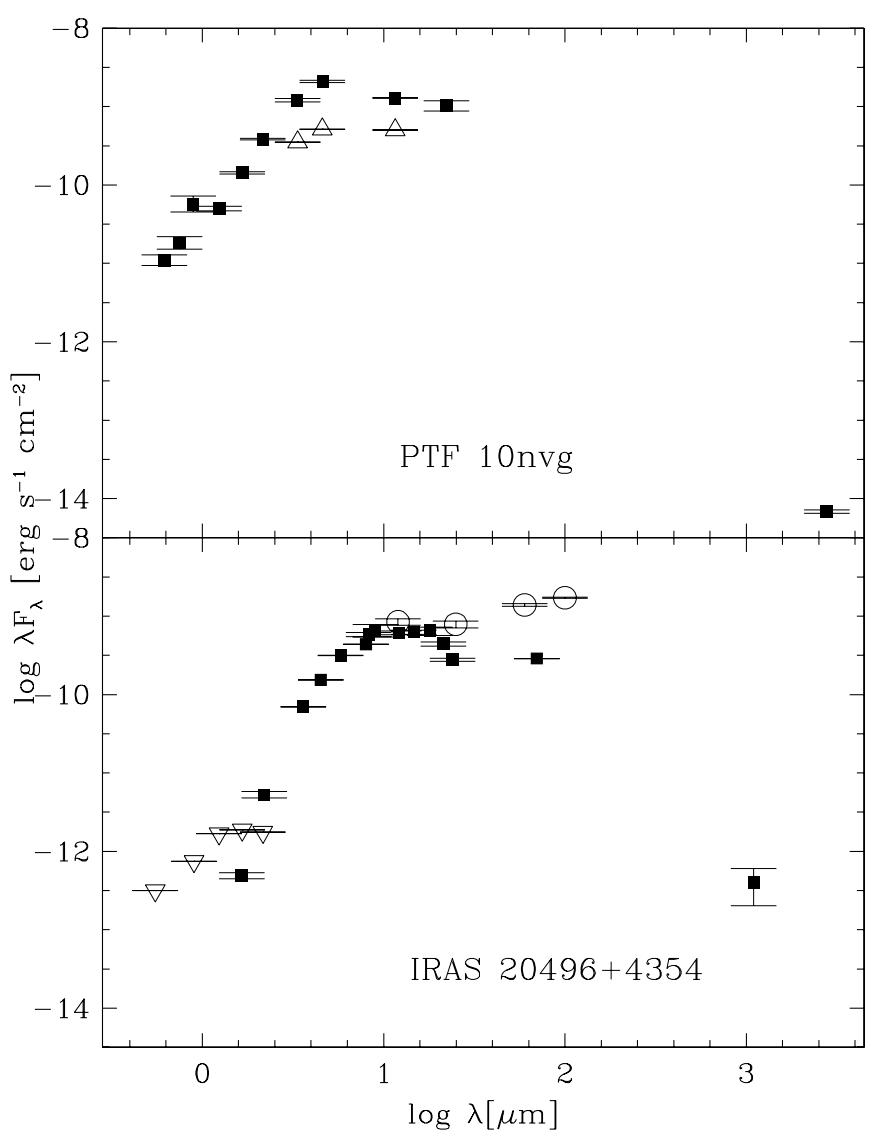

Figure 8. Spectral energy distributions updated from those presented in Covey et al. to include WISE and CARMA photometry. Bottom panel shows literature data from 1980-2009 while upper panel shows outburst photometry from 2010. Optical and near-infrared photometry is selected to be closest in time to plotted mid-infrared and millimeter photometry. At WISE wavelengths, both the saturated and the profile-fit-corrected magnitudes are shown. Downward facing triangles are upper limits and upward facing triangles are lower limits while squares and circles (large beam IRAS measurements in the left panel) are detections. As discussed in the text, the 2010 brightening of the source had mid-infrared as well as near-infrared and optical manifestations.

The post-outburst CARMA measurements at $2.7 \mathrm{~mm}$ can be compared with the pre-outburst data from the Bolocam Galactic Plane Survey at $1.1 \mathrm{~mm}$ (Rosolowsky et al. 2010) to search for variability at millimeter wavelengths. The Bolocam survey reported a $2 \sigma$ detection at the position of PTF 10nvg with an integrated flux of $148 \pm 74 \mathrm{mJy}$, although the integrated flux may be underestimated by $50 \%$ (Rosolowsky et al. 2010). The $1.1 \mathrm{~mm}$ detection is consistent with a point-source at the $33^{\prime \prime}$ angular resolution of Bolocam. The higher resolution CARMA $2.7 \mathrm{~mm}$ continuum image resolves the continuum emission into two compact sources and a diffuse extended component (see Figure 2). The integrated flux of these three components measured with aperture photometry is $11.3 \pm 1.4 \mathrm{mJy}$, where a $10 \%$ calibration uncertainty is assumed. While additional extended emission may be "resolved out" with CARMA, we nonetheless derive a spectral millimeter spectral slope $\left(F_{v} \propto\right.$ $\left.v^{\alpha}\right)$ of $\alpha=2.9 \pm 0.6$. Assuming the millimeter continuum emission is optically thin, the expected slope is $\alpha \approx 4$ for interstellar medium grains, and $\alpha \approx 3$ if grains have grown to approximately millimeter in size. Thus within the considerable measurement uncertainty, the observed spectral slope is consistent with that observed in circumstellar disks and envelopes, and we find no evidence for variation in the millimeter flux toward PTF 10nvg.

As demonstrated above, however, PTF 10nvg has shown significant variability at mid-infrared wavelengths. The $2-25 \mu \mathrm{m}$ spectral slope, originally defined for ground-based $K$-band and IRAS data by Lada (1987) as $\alpha=d \log \lambda F_{\lambda} / d \log \lambda$, can be computed in the pre-outburst and post-outburst stages. Using the data shown in Figure 8 and with the caveat that there are not ideal matches in the timing between available $K$-band and mid-infrared photometry, we indeed find a dramatic change in the spectral slope of the source from pre-outburst to postoutburst. Regardless of whether IRAS $25 \mu \mathrm{m}, M S X 21 \mu \mathrm{m}$, or Spitzer $24 \mu \mathrm{m}$ photometry (taken over a time span of three decades) is compared to the 2MASS $2 \mu \mathrm{m}$ upper limits from 2000 or the UKIDSS measurement from 2006, we find the pre2010 spectral slope is indicative of a "Class I"-type SED: specifically, we find $\alpha>2$ (in the range $>2.1$ to $>2.5$ ) when computed with the 2MASS upper limit, and $1.6<\alpha<2.0$ when computed from the UKIDSS measurement.

Computing an $\alpha$ value from the post-outburst PAIRITEL $K$-band observations and the similarly post-outburst WISE $22 \mu \mathrm{m}$ observations produces a much flatter spectral slope of $\alpha \sim 0.3$. This $\alpha$ value lies at the low end of the typically defined "Class I" range, but the dramatic change in spectral slope from the pre-2010 measurements indicates that, while PTF 10nvg demonstrably brightened by factors of several or more during and following the 2010 outburst, the near-infrared and optical brightening significantly exceeded that in the mid-infrared.

While the data in hand do establish that PTF 10nvg's preoutburst and post-outburst mid-infrared spectral slopes were notably different, we have little leverage for establishing the physical mechanism that drove the observed change. We consider three mechanisms: (1) direct extinction of the mid-infrared emitting region, as we invoke to explain much of the optical and near-infrared variability; (2) shadowing of the outer disk by a vertical perturbation in the inner disk; (3) an overall increase in the luminosity of the inner disk, due to internal or external heating related to an increase in the disk or stellar accretion rate. The current data do not clearly identify one of these possibilities as the favored interpretation.

1. Extinction. As noted above, PTF 10nvg's 2010 brightening included a four-fold increase in its $\sim 22 \mu \mathrm{m}$ flux. Interestingly, this change is equivalent to a difference of $1.5 \mathrm{mag}$ in extinction at this wavelength, and corresponds to a difference in $A_{V} \sim 30 \mathrm{mag}$ according to standard extinction laws. This is close to the maximum extinction change inferred from variations in the near-infrared colors (see Figures 6 and 9). Explaining the observed mid-infrared flux variability as an extinction event does require, however, the obscuration of a significantly larger portion of the inner disk than is required to explain the extinction experienced by the source's optical and near-infrared emission, which presumably arises directly from the central protostar and the inner disk wall. To understand the spatial scale an obscuring clump would need to possess to occult the full $22 \mu \mathrm{m}$ emitting region, we consulted a range of disk models calculated as in Isella et al. (2012) spanning a range of flaring angles $(\mathrm{h} / \mathrm{R})$. These models indicate that nearly all of the $2 \mu \mathrm{m}$ flux emits from the disk's innermost radii, $r<0.2 \mathrm{AU}$, with the bulk of it at $r<0.1 \mathrm{AU}$, regardless of geometry. The $22 \mu \mathrm{m}$ flux, by contrast, does show a greater dependence on the disk's flaring angle; for flat and moderately flared disks, the bulk of the $22 \mu \mathrm{m}$ flux arises 


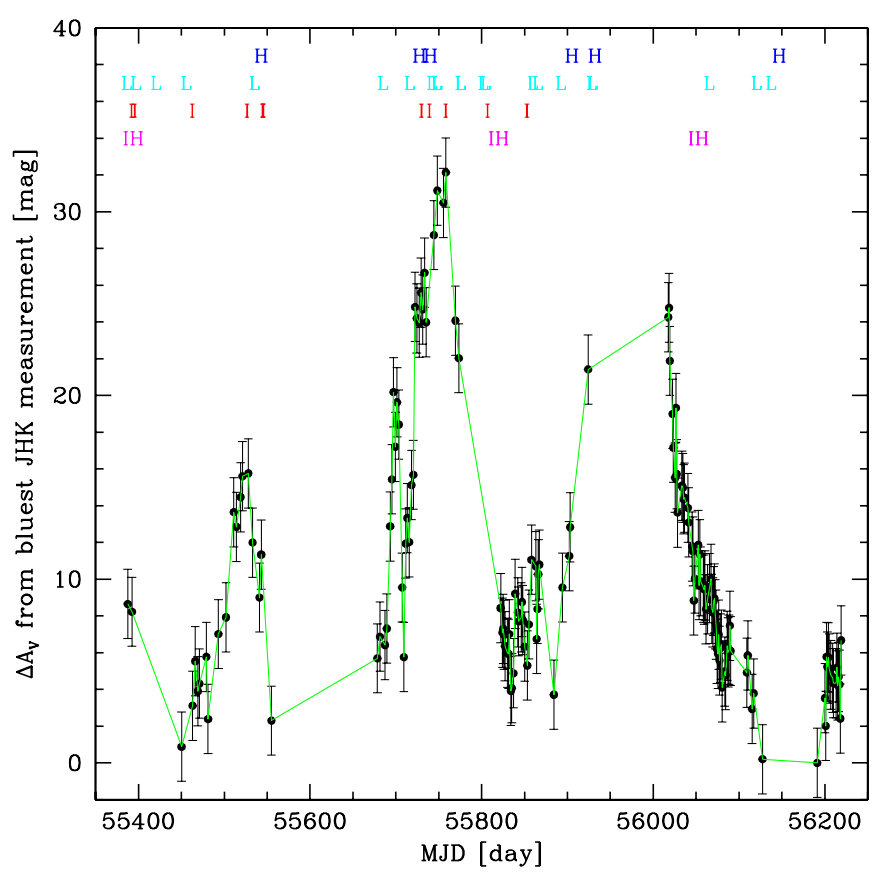

Figure 9. Differential optical extinction, $\Delta A_{V}$, measured from the color changes in Figure 6 relative to the bluest photometry captured in our near-infrared time series and an assumed extinction law. Error bars are calculated assuming the typical photometric errors in Table 3. The alphabetical labels indicate the timing of our spectroscopic follow-up of the 2010 brightening of PTF $10 n v g$, with " $H$ " representing high-dispersion optical data, "L" representing low-dispersion optical data, "I" indicating low-dispersion near-infrared data, and " $\mathrm{IH}$ " indicating high-dispersion near-infrared data, all as described in the text.

(A color version of this figure is available in the online journal.)

from the disk's inner $0.5-0.7$ AU region, within the radii implied for the location of the obscuring clump based on the characteristic timescale of the extinction events detected at shorter wavelengths. For disks with larger flaring angles, as might be expected for a typical Class I source, the $22 \mu \mathrm{m}$ emitting region extends over a larger radial range and well beyond $1 \mathrm{AU}$, requiring a larger obscuring clump to explain the observed mid-infrared variability.

2. Shadowing events. Models in which an increase in the apparent scale height of the inner disk (due either to, e.g., dynamical changes in the disk structure, or the apparent position with respect to the line of sight of a persistent orbiting perturbation) decreases the illumination of the outer disk have recently been developed to explain correlated near- and mid-infrared variability observed from young disked stars (Muzerolle et al. 2009; Espaillat et al. 2011; Flaherty et al. 2012). These models, by radiatively coupling the inner disk to more distant disk regions, provide a viable mechanism for inducing changes in the $24 \mu \mathrm{m}$ flux on timescales shorter than the local dynamical timescale. However, these models typically have been invoked to explain changes in $22 \mu \mathrm{m}$ flux at the $0.1-0.2 \mathrm{mag}$ level, not the 1.5 mag level observed from PTF 10nvg. In modeling midinfrared variability in T Tau S, van Boekel et al. (2010) note that detailed radiative transfer models of this mechanism suggest that mid-infrared brightness changes larger than 0.2 mag require remarkably large perturbations in the inner disk. These models also predict a "pivot point" at 5-10 $\mu \mathrm{m}$, where the spectral changes on either side of the pivot are anti-correlated, due to the increased near-infrared flux emit- ted by the inner disk as it shadows the outer disk, and vice versa. The brightening exhibited by PTF 10 nvg provides no evidence for such a pivot point, but given the numerous components which are likely contributing to PTF 10nvg's spectral evolution, and the lack of simultaneous data over a broad wavelength range, the existence of a $\sim 8 \mu \mathrm{m}$ pivot point cannot be ruled out either.

3. Accretion-related increase in disk luminosity. If PTF 10nvg's brightening is associated with a major accretion outburst, the increase in external illumination from the stellar accretion shock and/or a similar increase in viscous heating due to material flowing through the circumstellar disk could lead to a significant increase in the system's mid-infrared flux. This mechanism has provided the foundation for increasingly detailed models of the spectral morphology of FU Ori objects, over a range of wavelengths from the optical into the far-infrared (Zhu et al. 2008), and is also consistent with observations of the wavelength dependent timescales for the decay of the near- and mid-infrared emission from V1057 Cyg, a post-outburst FU Ori object (Simon \& Joyce 1988). Intriguingly, van Boekel et al. (2010) also demonstrate that their models of accretion-driven disk emission predict near- and mid-infrared color-magnitude changes that are broadly consistent with expectations from standard extinction models: if true, this suggests that even simultaneous broadband photometry spanning the near- to mid-infrared during a distinct brightening and fading event may not suffice to establish the true nature of PTF 10nvg's mid-infrared variations.

\section{SPECTROSCOPIC ANALYSIS}

Our new optical and near-infrared spectra at low dispersion can be considered along with the spectroscopic data published by Covey et al. that sampled the source both before and after its absolute brightness maximum in 2010 August. The 2011 and 2012 spectra sample epochs in the light curve of PTF 10 nvg when the source was near local maxima in brightness as well as when it was deep in extended faint states, e.g., during summer/fall of 2011 and fall/winter of 2012 (Figure 1). The high-dispersion spectra also sample the light curve at relatively bright as well as much fainter photometric states.

Figure 9 illustrates the ensemble of spectral epochs relative to the estimated optical extinction variation over the time series. The latter comes from calculating the color excess from the bluest observed near-infrared color and applying the Indebetouw et al. (2005) extinction law. We note the high density of spectral coverage obtained during the faintest, reddest state of the PTF 10nvg light curve in 2011, summer. Given the sparse spectral sampling we do not consider the evidence for spectroscopic periodicity. However, we do observe a seemingly repeating pattern in several spectroscopic signatures. Specifically, the forbidden lines are most prominent during the high-extinction epochs represented in Figure 9. Further, spectra taken when PTF 10nvg was in one photometric cycle are very similar to those taken in earlier photometric cycles at similar phase; for example, repeatedly when brightening, the forbidden lines are clearly present but quite weak relative to their dominance in the faint photometric states.

Our discussion of the spectral time-series data is divided into the presentation of the continuum behavior (illustrated for the optical in Figure 10 and for the infrared in Figures 11 and 12) in Section 5.1, and then a description of the lines, as illustrated 

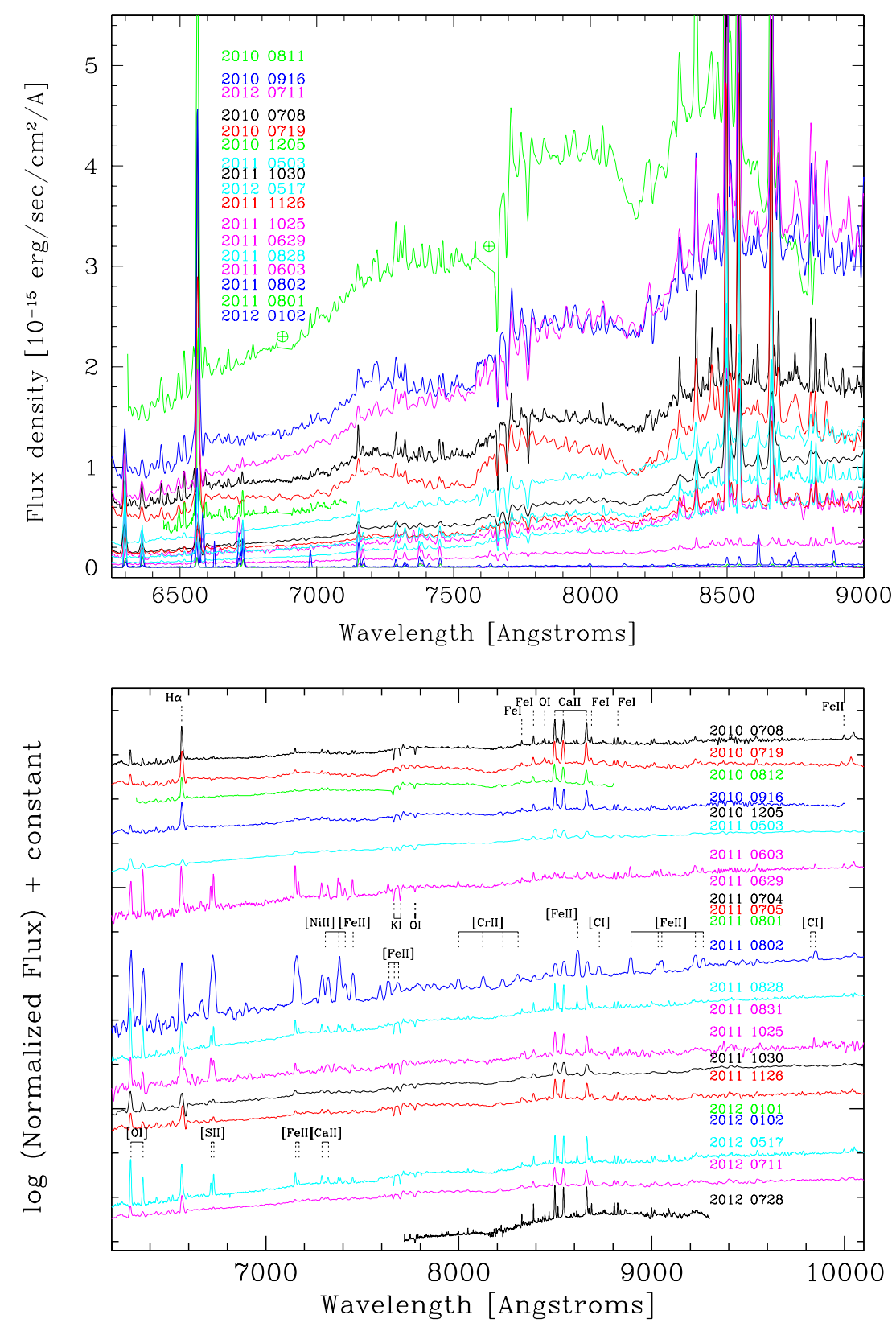

Figure 10. Top panel: flux-calibrated optical spectra plotted to highlight variable continuum brightness. Data from 2011 July 4 and July 5 , and from 2012 January 1 are excluded due to significant noise. The spectrum from 2010 August 11 is not corrected for telluric absorption (the prominent atmospheric $B$ and $A$ bands have been smoothed over). The spectrum from 2010 August 12 is not shown but has 2.5 times the flux of the previous night's spectrum, consistent with the light curve in this period. Bottom panel: normalized spectral time series, plotted on a log scale to emphasize variable emission line strengths. Unmarked weaker emission lines are generally low-excitation transitions of Fe I or Fe II. Note the slightly wider spectral range than in the top panel, so as to feature additional forbidden lines. Spectra are ordered by date; some dates have no accompanying spectra plotted due to significant continuum noise or, in the case of 2010 August 12 , redundancy with an adjacent spectrum. During brighter photometric states the $\mathrm{H} \alpha$ and $\mathrm{Ca}$ II lines are prominent, while in fainter states, the forbidden emission lines are more prominent, as also shown in Figures 13 and 14.

(A color version of this figure is available in the online journal.)

in Figures 13-19. The line discussion is subdivided into windindicating absorption lines in Section 5.2 and then accretionand outflow-diagnostic emission lines in Section 5.3. The various emission species are further separated into molecular (Section 5.3.1), permitted atomic (Section 5.3.2), and forbidden atomic (Section 5.3.3) lines. Evidence for spatially extended forbidden line emission is presented in Section 5.3.4. A wide range of thermal and mechanical processes are probed with the array of available spectral diagnostics. The molecular lines measure gas having temperatures up to several thousand kelvin. The atomic lines diagnose temperatures usually in the range $\sim 5000-20,000 \mathrm{~K}$ (but up to $40,000 \mathrm{~K}$ ), and densities $n_{e} \approx$ $10^{4}-10^{9} \mathrm{~cm}^{-3}$ (forbidden lines) and $n_{\mathrm{H}} \approx 10^{9}-10^{12} \mathrm{~cm}^{-3}$ (permitted lines). Our main focus is on describing the evolution in time of the line presence, line ratios, and line profiles in the context of mass accretion and outflow.

\subsection{Evolution of the Optical and Infrared Spectral Continuum}

The large-amplitude photometric changes exhibited by PTF 10 nvg are mirrored by large changes in the spectral continuum. 

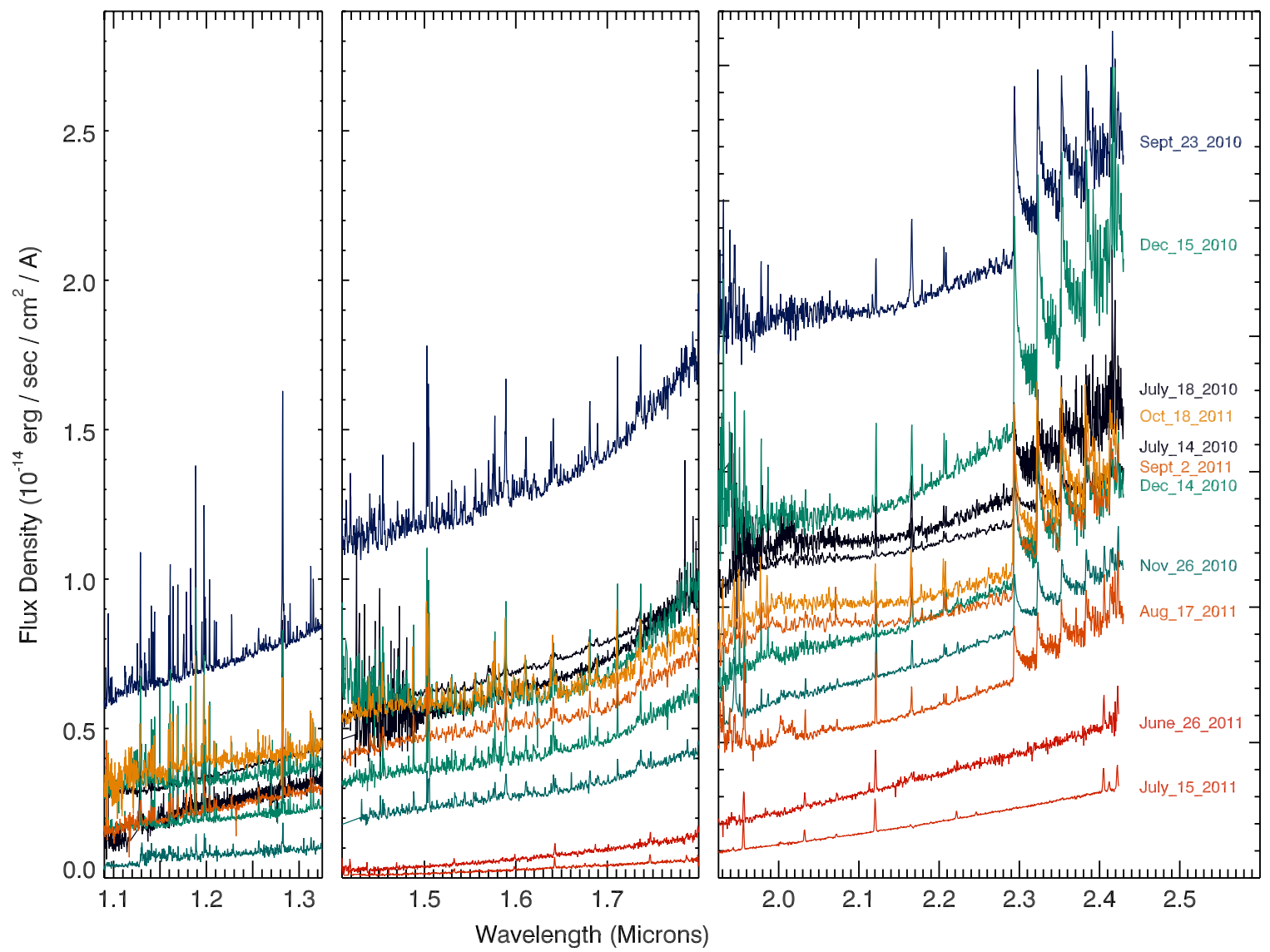

Figure 11. Flux-calibrated near-infrared spectra of PTF 10nvg. While the absolute flux densities observed in the time series vary by a factor of $\sim 10$, once the spectra have been corrected with a standard $R_{V}=3.1$ extinction law for the photometrically derived reddening estimates shown in Figure 9 , the spread in flux reduces to a factor of two-within expectations given the non-simultaneity of the spectra and the photometric extinction estimates. Spectra obtained on 2011 June 26 and July 15 , when PTF 10nvg was in a particularly faint state, lack the numerous atomic (e.g., $\mathrm{H}, \mathrm{Na}, \mathrm{Ca}, \mathrm{Mg}$, etc.) and molecular (e.g., $\left.\mathrm{CO}, \mathrm{H}_{2} \mathrm{O}\right)$ emission features that dominate the brighter spectra. The few emission features that remain visible in the faint state (e.g., $\mathrm{H}_{2}$, [Fe II]) likely arise in the jet/outflow.

(A color version of this figure is available in the online journal.)

This is illustrated in Figure 10 showing the time series of optical spectroscopy within the $R$ - and $I$-band regions and in Figure 11 showing the infrared spectra in the $Y_{-}^{-}, J_{-}, H_{-}$, and $\mathrm{K}$-band atmospheric windows.

Flux-calibrated optical spectra (top panel in Figure 10) exhibit spectral continuum slope changes that accompany the photometric brightness changes, as discussed in Covey et al. Notably, the $\mathrm{TiO} / \mathrm{VO}$ emission features (which are broad in wavelength and thus act like a continuum) have varied in strength relative to the nearby out-of-band continuum regions. Normalized optical spectra (bottom panel of Figure 10) illustrate line-to-continuum changes. During epochs when the source was fainter the signal-to-noise ratio is lower, but this does not preclude the detection of numerous prominent forbidden lines that are not seen in the brighter photometric states when the continuum emission is stronger. Conversely, when the source is bright, permitted lines are more prominent than forbidden lines. We discuss these and other spectral line changes in more detail in Section 5.3.

The infrared spectral continuum also exhibited differential brightening and fading. Along with the time series shown in Figure 11, we highlight the near-infrared spectra from the brightest and the (nearly) faintest epochs in Figure 12. Variations in PTF 10nvg's near-infrared spectral continuum appear consistent with the extinction variations discussed above based on the near-infrared photometric monitoring data, and illustrated in Figures 6 and 9. To verify this assertion, we used an $R_{V}=3.1$ Fitzpatrick (1999) extinction law ${ }^{14}$ to de-redden each moderate resolution near-infrared spectrum according to the photometrically derived $A_{V}$ estimates in Figure 9, as interpolated onto each spectroscopic epoch. While the flux densities of the observed spectra differ by more than an order of magnitude, the flux densities of the de-reddened spectra agree to within a factor of $\sim 2$. Similarly, the spectral slopes of the de-reddened spectra also agree well generally, with the exceptions of the spectra acquired on 2010 November 26, 2011 June 26, and 2011 July 15, when the source was deep in (two different) photometric minima. Considering the uncertainties imposed by the non-simultaneity of the spectra and the photometric data from which the extinction estimates are derived, this level of agreement suggests that extinction variations account for much of the near-infrared photometric and spectroscopic continuum variability within our time-series data.

\footnotetext{
14 The Fitzpatrick (1999) reddening prescription (employed here to the spectra because it is smoothly varying with wavelength) and the Indebetouw et al. (2005) extinction (used earlier with the photometry since it is determined empirically using the same filters) are the same to within 5\% in the near-infrared; this difference is roughly the same as the estimated error in the flux calibration of our near-infrared spectra.
} 


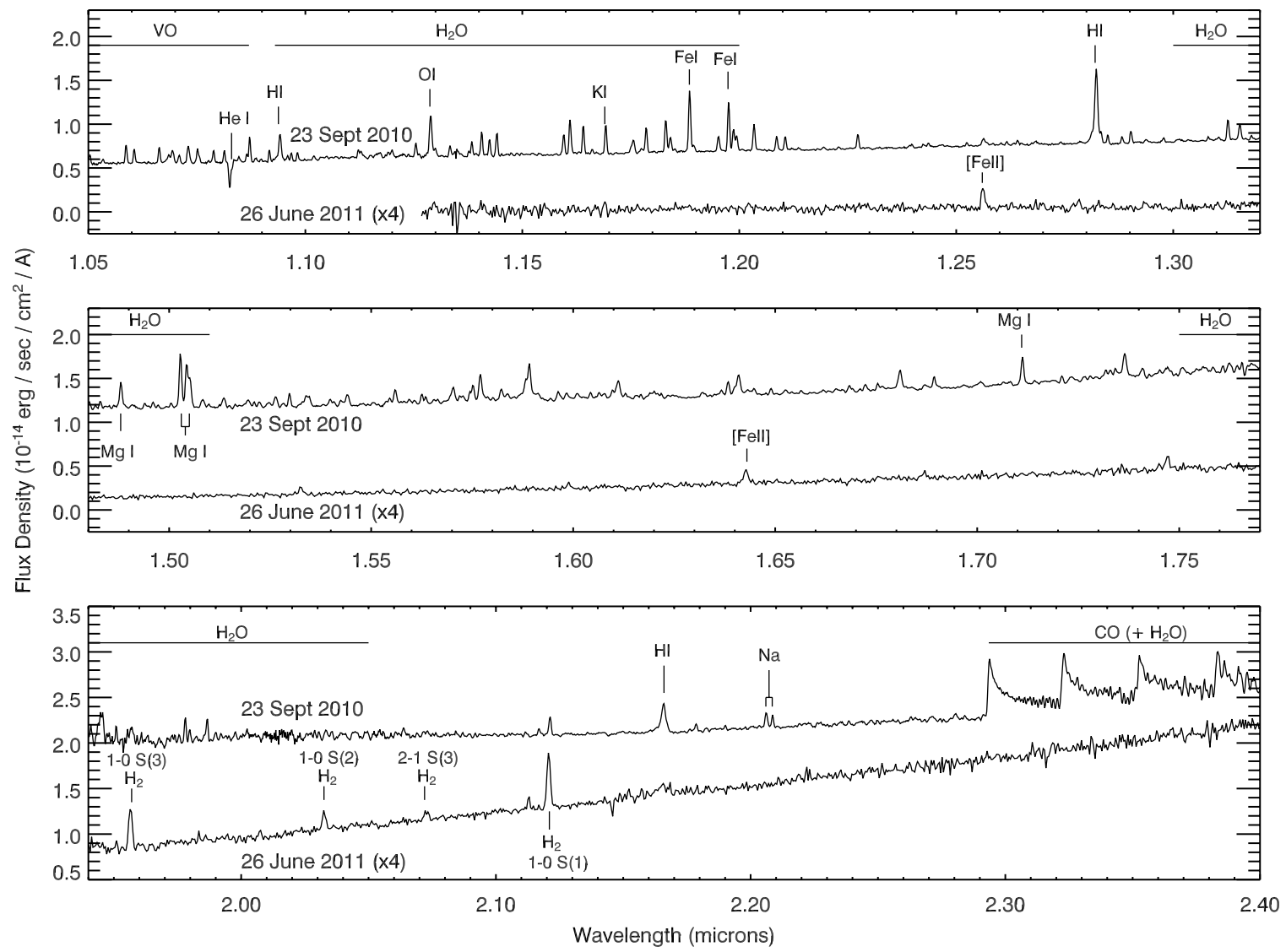

Figure 12. Comparison of PTF 10nvg near-infrared spectra during the brightest (2010 September 23) and second faintest (2011 June 26, adopted because it is morphologically similar to the faintest 2011 July 15 spectrum but extends to bluer wavelengths) epochs captured in our moderate resolution near-infrared spectral monitoring. Numerous permitted atomic lines (e.g., Fe I cluster at $\sim 1.15-1.2 \mu \mathrm{m}$ ) are prominent in the brighter spectrum but absent in the fainter spectrum, which exhibits primarily $\mathrm{H}_{2}$ and $[\mathrm{Fe} \mathrm{II}]$.

\subsection{Evolution of the Wind-indicative Absorption Lines}

Our spectra contain a number of lines sensitive to outflowing material. The early optical spectrum of PTF 10nvg shown by Covey et al. exhibited only the following lines in absorption Na I D, K I $\lambda \lambda 7665,7699$, and Oг $\lambda 7774$, all with blueshifted velocities relative to the expected line center wavelengths. These are either resonance lines or lines having a metastable lower level, so they readily go into absorption. Absorption was seen against the broad $\mathrm{TiO} / \mathrm{VO}$ molecular emission continuum mentioned above, and there were no emission components to the profiles. As the source varied photometrically over the 2010, 2011, and 2012 seasons, the blueshifted absorption remained relatively constant with respect to a normalized continuum, i.e., in equivalent width; values hovered around $2-3 \AA$ over most of this time period (though possibly somewhat higher, $3-4 \AA$, toward the end of 2011). Between 2011 June 3 and 29, these absorptions disappeared as the continuum faded; this could be as they became undetectable in the lower signal-to-noise ratios characteristic of these spectra, or perhaps intrinsically if the wind strength decreased. By 2011, late August, when the source was again near a peak in brightness, line absorption from winds was again apparent at the same equivalent width level and is seen in all subsequent "bright-state" spectra. The lack of change in absorption equivalent width is consistent with the extinction variation interpretation of the continuum behavior, implying that the large extinction variations arise exterior to the wind absorption region.
At high-dispersion, the bright-state data (e.g., in Figure 15) show that the $\mathrm{Na}$ I $\mathrm{D}$ doublet absorption is saturated at line center, with a boxcar like profile at zero flux extending blueward to $\sim-275 \mathrm{~km} \mathrm{~s}^{-1}$. The $\mathrm{K}_{\mathrm{I}} \lambda \lambda 7665,7699$ doublet also has a broad blueshifted absorption profile, extending from approximately -50 to $-250 \mathrm{~km} \mathrm{~s}^{-1}$. Its depth is approximately $40 \%$ of the continuum level, shallower than the saturated Na I D doublet, absorption. Both lines have, in addition to their broad blueshifted absorptions, a very narrow, very slightly blueshifted absorption component. ${ }^{15}$ The Oг $\lambda 7774$ triplet absorption profile is hard to decipher due to the component blending, but it appears approximately neutral in velocity and extends to approximately $60 \%$ of continuum depth; approximately $130 \mathrm{~km} \mathrm{~s}^{-1}$ of the line width is due to the triplet blending. We note that it is claimed in Aspin (2011) that the $\mathrm{O}$ I $\lambda 8446$ line also exhibited a blueshifted absorption profile in 2010 September, but we see only emission from our low-resolution data taken in August and September (Figure 10) and in our high-resolution data taken in December (Figure 15) of that year.

The time evolution of the optical wind-indicative absorption lines cannot be well documented given that source faintness precluded the detection of continuum and thus any absorption against it in many of our high-dispersion spectra. In the HIRES

\footnotetext{
15 While this might be attributed to an interstellar contribution, the appearance of the low-velocity narrow $\mathrm{K} I$ and narrow $\mathrm{Na}$ I is similar to a non-interstellar low-velocity narrow component in the He I $\lambda 10830$ profile discussed below. In the bright state, there is also a narrow absorption at this velocity in $\mathrm{H} \alpha$ profiles (see Figure 16).
} 


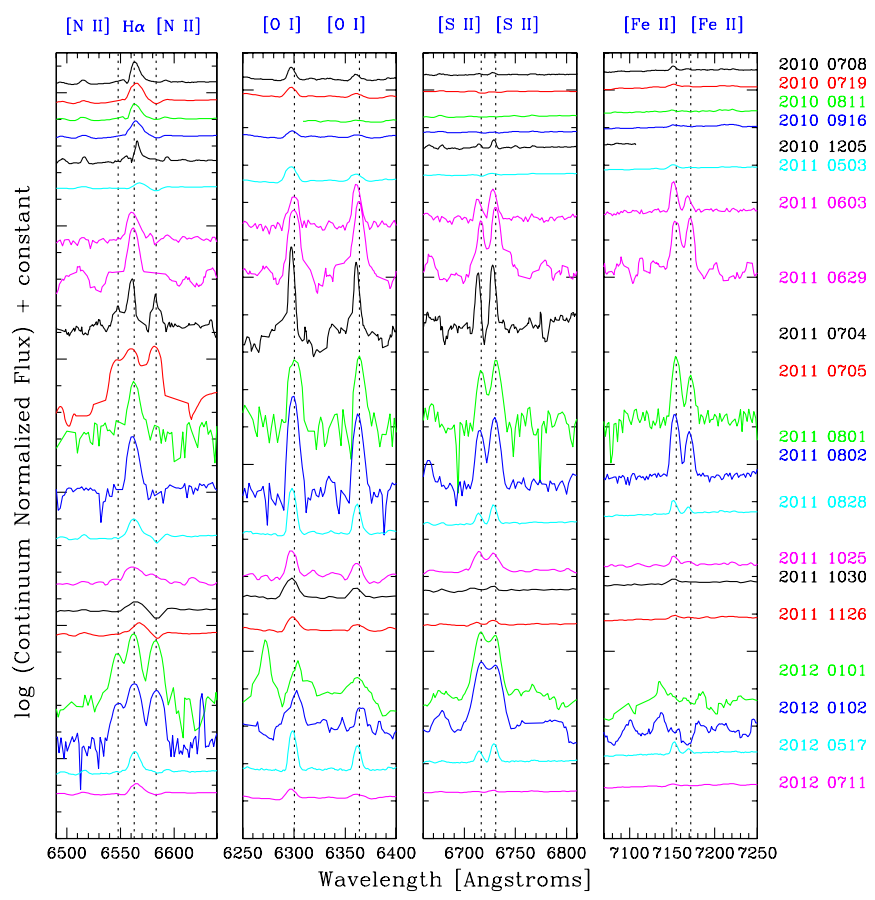

Figure 13. Time-series optical spectra in the same color scheme and order as the bottom panel of Figure 10, showing $\mathrm{H} \alpha$ as well as the [N II], [OI], [S II], and [Fe II] forbidden line doublets. Lower signal-to-noise spectra have been smoothed. Given the large variations in line-to-continuum ratio over the time series, the ordinate is on a log scale. Note the variation in the doublet line ratios and the slightly blueshifted line centers which are apparent even at low resolution.

(A color version of this figure is available in the online journal.)

data from 2011 December, however (taken after the source had undergone several episodes of photometric fading and brightening), the $\mathrm{K}_{\mathrm{I}} \lambda \lambda 7665,7699$ doublet and the NaI D doublet absorption profiles appear to have approximately the same form and terminal velocities as in the 2010 December bright-state spectrum. Another bright-state HIRES spectrum from 2012 August shows roughly similar K I and Na I features, but even higher velocity blueshifted absorption in the O I $\lambda 7774$ line-out to $-250 \mathrm{~km} \mathrm{~s}^{-1}$ compared to only -150 in Figure 15 . This broadening of blueshifted absorption by mid-2012 is also apparent at $\mathrm{H} \alpha$; see below.

Edwards et al. (2006) discussed the utility of the He I $\lambda 10830$ line (also with a metastable lower level) as a wind indicator, demonstrating that its sub-continuum absorption over a range of velocities probes both disk winds and stellar/polar winds in young stars. Kwan \& Fischer (2011) derived the physical conditions traced by He I (density $n_{\mathrm{H}} \sim 10^{9} \mathrm{~cm}^{-3}$, temperature $T \sim 10,000 \mathrm{~K}$, excitation through UV photoionization) as well as other accretion/outflow tracing lines. Kurosawa \& Romanova (2012) demonstrated in "observation" of MHD accretion/outflow radiative transfer models the sensitivity of this line to the high-energy radiation field, namely, $L_{X}$ and $T_{X}$, and were able to produce line profiles having redshifted and blueshifted absorption and emission features consistent with observations of young accreting stars. The early infrared spectrum of PTF 10nvg shown in Covey et al. exhibited only $\mathrm{He}$ I $\lambda 10830$ in absorption. The line was blueshifted relative to line center. In the left panel of Figure 17 we show the time-series behavior of this line at low dispersion. While the range of resolutions of the spectra in Figure 17 do influence the details of the observed profile somewhat, the He I line appears

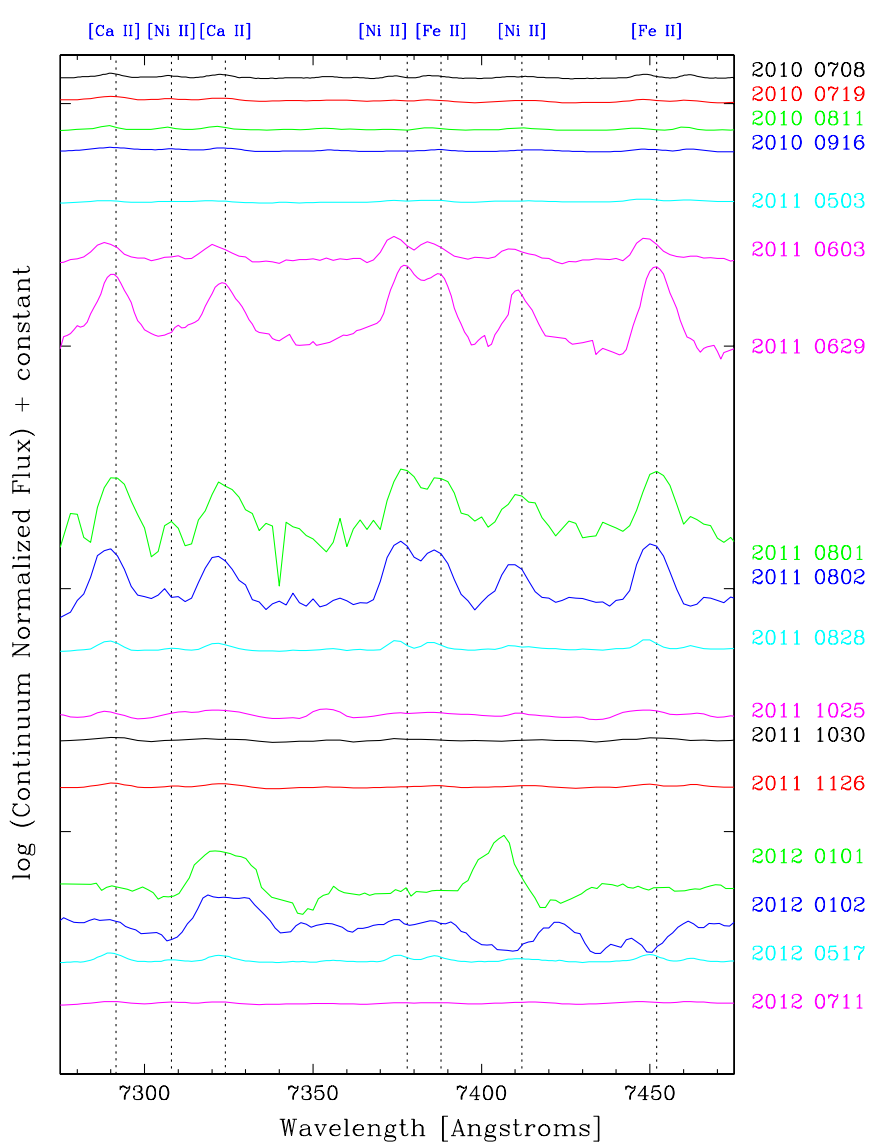

Figure 14. Time-series spectra in the 7275-7474 $\AA$ region showing the [Ca II] doublet as well as [Ni $\mathrm{II}]$ and [Fe II] lines that are prominent during faint photometric states. Lower signal-to-noise spectra have been smoothed, and neither of the 2011 July 4 or 5 spectra are shown.

(A color version of this figure is available in the online journal.)

to strengthen systematically over time, independent of the photometric state of PTF 10nvg. The line increases from $\sim 3 \AA$ in equivalent width and a maximum depth of $60 \%$ of the continuum in 2010 July to $\sim 8 \AA$ equivalent width and a maximum depth of $\sim 30 \%$ of the continuum in 2011 October.

These dramatic changes are also evident in the highdispersion He I $\lambda 10830$ profiles shown in Figure 18. The NIRSPEC spectra were obtained in 2010 July, when PTF 10nvg was near its absolute brightness maximum (which occurred in 2010 August), in 2011 September, when the source was in its prolonged optical minimum state, and in 2012 May as the source was rising toward a photometric peak. The change exhibited is consistent with the trend observed in the lower-resolution spectra. The faint-state 2011 spectrum is close to a classical P Cygni profile, with some sub-structure in each of the redshifted emission and the blueshifted absorption components, along with a narrow, slightly blueshifted absorption. This low-velocity narrow absorption component to the He $\mathrm{I} \lambda 10830$ profile is present in all three spectra and may be the same as that seen in the $\mathrm{K}_{\mathrm{I}}$ and $\mathrm{Na}$ I profiles. In both brighter state spectra, the redshifted emission component at about $+50 \mathrm{~km} \mathrm{~s}^{-1}$ that was present in the faint state is gone. However, the two brighter state spectra are different in their broad blueshifted absorption components. Relative to the 2010 bright-state spectrum, the 2011 faint-state and 2012 bright-state blueshifted absorptions both are deeper $(25 \%$ of continuum versus $60 \%$ of continuum) and broader (about -100 to $-300 \mathrm{~km} \mathrm{~s}^{-1}$ versus -50 to $-250 \mathrm{~km} \mathrm{~s}^{-1}$ ). 
In summary, the blueshifted absorption exhibited in the Na I D, K I $\lambda 7665,7699$, Ог $\lambda 7774$ lines and He I $\lambda 10830$ line, as well as blueshifted absorption components to the broad $\mathrm{H} \alpha$ and $\mathrm{Ca}$ II $\lambda \lambda 8498,8542$, and 8662 emission profiles (discussed further in Section 5.3 below) are indicative of a strong outflow/wind. The absorption must occur in a region outside of the optical and near-infrared continuum in the case of the pure absorption features, and outside the region producing the broad $\mathrm{H} \alpha$ and $\mathrm{Ca}$ II line emission (mainly the accretion column but also the wind itself).

\subsection{Evolution of the Accretion and Outflow Diagnosing Emission Lines}

At low resolution, Figures 13 and 14 show detail relative to Figure 10 of some of the optical emission line behavior of PTF 10nvg during 2010 and 2011. Among the rich optical emission line spectrum are permitted atomic species of $\mathrm{H}$ I, O I, Fe I, Fe II, $\mathrm{Ca}$ I, and Ca II. Notably, there is no evidence of optical He I. There are also (strong) forbidden emission lines in the optical from [C I], [O I], [Fe II], [Ni II], [Cr II], [Ca II], and [S II]. Several of these species are rarely seen in young star spectra, even those which are emission line dominated. Figure 17 illustrates the infrared time series of emission line behavior. In the infrared, we see permitted atomic species of Al I, Ca I, Fe I, K I, Mg I, Na I, $\mathrm{O}$ I, Si I, and Ti I as well as forbidden [Fe II], [N I], and [S II].

In both the optical and infrared, there was notable weakening of permitted atomic line emission when the continuum faded. The forbidden atomic line species, on the other hand, became significantly more prominent in the faint-state spectra; this prominence is due not only to better contrast relative to the continuum as it faded, but also to variation (by up to a factor of two in both the optical and near-infrared) in the forbidden line absolute fluxes. Infrared $\mathrm{H}_{2}$ lines behaved in a manner similar to the forbidden atomic lines. As the permitted atomic emission is generally associated with the accretion flow (with some lines influenced by outflow physics), and the forbidden atomic and the $\mathrm{H}_{2}$ lines are generally associated with outflow, we suggest below that their respective correlation and anti-correlation with the photometric state of PTF 10nvg implies that the forbidden and $\mathrm{H}_{2}$ lines are formed outside the extinction region. Other molecular lines, notably infrared CO and optical TiO/VO, however, followed the emission behavior of the permitted atomic lines, suggesting that these lines are also formed in the inner disk region.

At high dispersion, the emission line spectrum of PTF 10nvg bears significant resemblance to that of V1331 Cyg in terms of the species present and the relative line strengths. Direct comparison of small portions of the red optical and of the $1 \mu \mathrm{m}$ region high dispersion spectra are provided in Figure 19. The PTF 10nvg spectrum is more "extreme" in terms of emission line presence and strength than the spectra of other well-known "continuum plus emission" sources. Specifically, compared to DR Tau, Z CMa, or SVS 13, for example, PTF 10nvg exhibits a larger number of emission line species and with larger blueshifts. However, at $1 \mu \mathrm{m}$ the blueshifted absorption trough in He I $\lambda 10830$ for PTF 10nvg (Figure 18) is not as deep or as wide as in these objects (see Figure 1 of Edwards et al. 2003 for profiles) despite the similar line terminal velocities. V1331 Cyg similarly has a He I profile that is much deeper and broader than that of PTF 10nvg. But unlike the spectra of the above sources around $1 \mu \mathrm{m}, \mathrm{V} 1331$ presents a similarly rich emission line spectrum as PTF 10nvg in both the permitted and some of the forbidden line species (bottom panel of Figure 19). In the optical, the PTF 10nvg spectrum (from 2010 December, the only epoch in our high-dispersion data set that is not limited by signal-to-noise) is again well-matched to V1331 in the permitted line emission (top panel of Figure 19) though PTF 10nvg has stronger and more blueshifted forbidden line emission.

In the subsections below we discuss the details and timeseries behavior of the molecular (Section 5.3.1), permitted atomic (Section 5.3.2), and forbidden atomic (Section 5.3.3) lines. Summarizing our findings, the evolution of PTF 10nvg's spectroscopic properties support our interpretation from the photometry that the source undergoes large variations in lineof-sight extinction $\left(A_{v}>30^{m}\right)$ toward the origin of the optical/near-infrared continuum and permitted emission lines. Because emission equivalent width increases (decreases) with continuum fades (rises) as $10^{0.4 \times \delta m}$, we do expect some evolution in line strength. However, the emission lines which are thought to arise from shocked circumstellar material do not appear to be subject to the bulk of these extinction variations; the fluxes of these lines are relatively stable throughout the monitoring period, and therefore appear significantly more prominent at epochs when the other components of the spectrum appear heavily extincted.

\subsubsection{Molecular Emission}

Above, when describing the behavior of PTF 10nvg's optical continuum, we discussed that the broad $\mathrm{TiO}$ and $\mathrm{VO}$ molecular emission at optical wavelengths is time variable. To quantify the effect, we measured the strength of the $\mathrm{TiO}$ emission at 6250 , $6760,7100,7800,8455$, and $8880 \AA$ as well as the VO emission at 7445 and 7865 using band indices as described in Hillenbrand (1997). There is stronger optical molecular emission relative to the continuum during the 2010 outburst and in early 2012 when the source was bright, relative to the periods when the source was fainter, notably in late 2011. This is consistent with the inference drawn from direct examination of the spectra both on an absolute scale and on a continuum-normalized scale (see Figure 10). In addition to the direct correlation between strength of the different molecular bands, they are all stronger when the permitted atomic emission is stronger. $\mathrm{TiO} / \mathrm{VO}$ emission is correlated as well with the optical spectral continuum slope.

Molecular emission is also seen in PTF 10nvg's near-infrared spectra, specifically in bands arising from warm $\mathrm{H}_{2} \mathrm{O}, \mathrm{CO}$, and VO (Figure 12). These molecular bands are all prominent in PTF 10nvg's bright states and all weaken relative to the continuum as the continuum fades. As Figures 12 and 17 demonstrate, the strength of the $\mathrm{CO}$ and $\mathrm{H}_{2} \mathrm{O}$ emission features are highly correlated with both emission from permitted atomic lines ( $\mathrm{HI}, \mathrm{Na}$ I, Ca I, etc.) and with the brightness of the underlying continuum.

Like the optical $\mathrm{TiO}$ and $\mathrm{VO}$ bands, the infrared $\mathrm{H}_{2} \mathrm{O}$, $\mathrm{CO}$, and $\mathrm{VO}$ bands are commonly seen in absorption in M-type photospheres. CO overtone emission is not uncommon in embedded protostars. For example, Doppmann et al. (2005) and Connelley \& Greene (2010) detected CO emission from $\sim 15 \%$ of the Class I and $\sim 25 \%$ Class I/II (i.e., flat spectrum) sources in their samples. Detailed analyses of the kinematic profiles of these $\mathrm{CO}$ band heads in several particularly well-studied $\mathrm{CO}$ emission sources suggest that the emission arises from the innermost regions of circumstellar disks in Keplerian rotation (e.g., Najita et al. 1996). The prominence of several oxygen species molecular lines when PTF 10nvg is brighter could be due to simple heating and cooling related to variation in optical and higher energy photon flux, or perhaps to molecule formation 


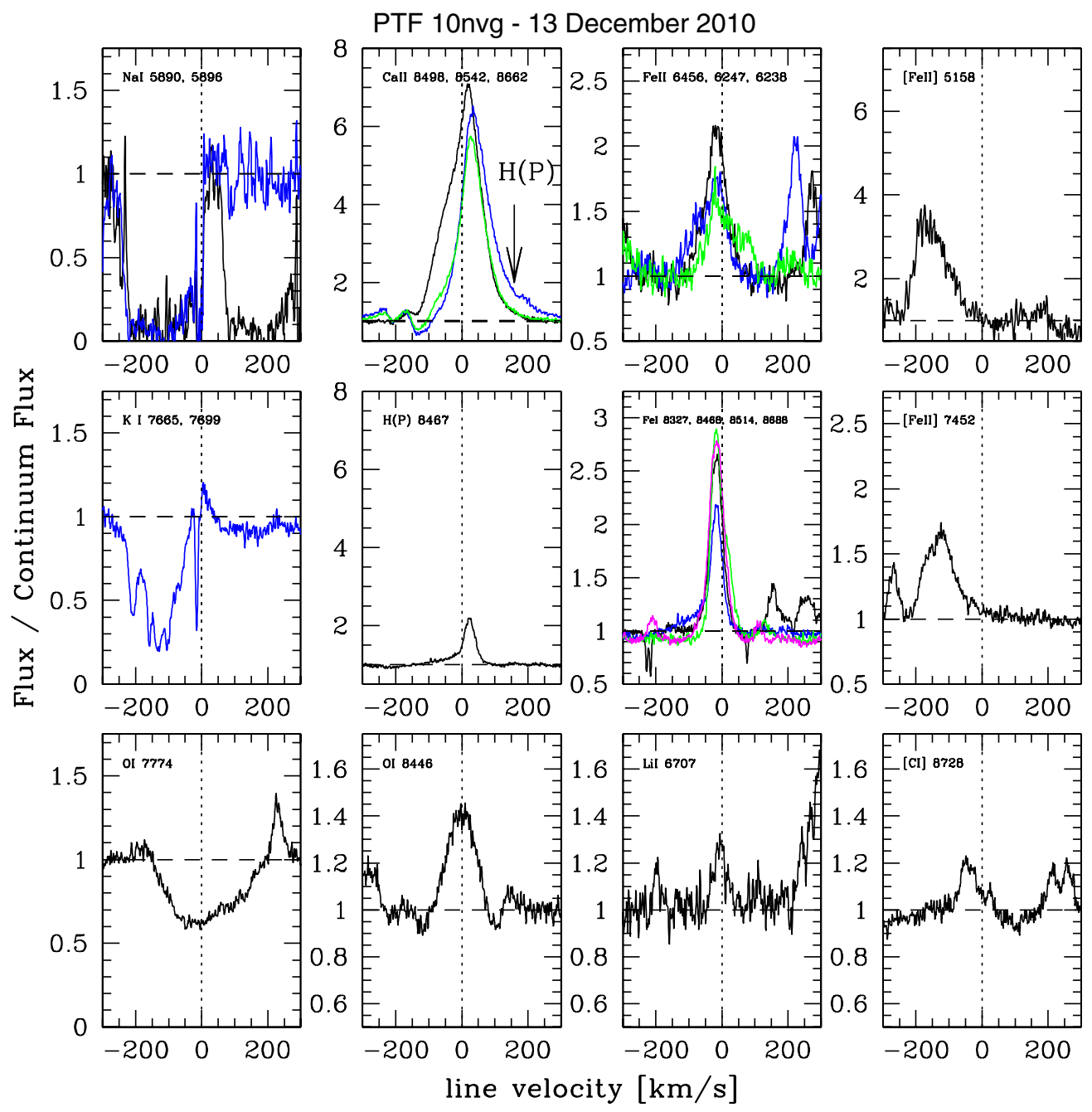

Figure 15. Optical line profiles from 2010 December with vertical dotted lines indicating zero velocity and the abscissa covering -300 to $+300 \mathrm{~km} \mathrm{~s}{ }^{-1}$. In each panel, the black profile represents the line indicated first in the panel legend; blue/green/magenta profiles, if present, indicate the second/third/fourth line in the panel legend (for example, in the Ca II panel the black, blue, and green profiles are those of the $\lambda \lambda 8498,8542$, and 8662 transitions). The Ca II triplet lines are broader than other atomic emission lines and likely have both accretion-related and outflow-related components; note that the red sides of these lines are each contaminated by H I Paschen lines, with the panel immediately below the Ca II panel showing another Paschen line on the same scale. Highly and moderately blueshifted emission is apparent in forbidden [Fe II] and [C I], respectively (see also Figure 16). In contrast, permitted Fe I, Fe II, and O I emission is centered at low blueshifts or nearly zero velocity with a range in profile widths including rather narrow profiles. Blueshifted absorption is seen in the wind-sensitive absorption lines of K I and Na I, with the blue sides of the Ca II triplet lines also exhibiting some (sub-continuum) absorption. The O I $\lambda 7774$ triplet is seen in absorption as well. Unusually, Li I $\lambda 6707$ appears to be in emission (see the text).

(A color version of this figure is available in the online journal.)

following dissociation during the bright state; see Hillenbrand et al. (2012) for further discussion. Alternatively, the correlation could be caused by obscuration of the molecular emitting region during the faint stages; this is the same explanation we invoke for the time variable behavior of the atomic line emission.

Molecular emission from $\mathrm{H}_{2}$, however, displays times series behavior with the opposite trend from the oxygen species (TiO, $\mathrm{VO}, \mathrm{CO}$ ). While $\mathrm{H}_{2}$ lines are present at all epochs, these lines appear most prominent during PTF 10nvg's faint states. The absolute line flux remains relatively constant, however, indicating that the increased prominence of these features during faint states is due to improved contrast with the nearby continuum. This behavior is qualitatively visible in Figure 17, where the proximity of the $2.12 \mu \mathrm{m} \mathrm{H}_{2}$ line to the $2.166 \mu \mathrm{m} \mathrm{H}$ I line provides a clear demonstration that $\mathrm{H}_{2}$ strengthens relative to $\mathrm{HI}$ as the extinction (as inferred from near-infrared colors) increases. This behavior strongly suggests that PTF 10nvg's variable extinction obscures the emission region responsible for the $\mathrm{HI}$ (and other permitted atomic emission and molecular emission) lines, but not the emission region from whence the $\mathrm{H}_{2}$ emission arises. To test this expectation in more detail, we compare in Figure 20 the ratio of the $\mathrm{H}$ I and $\mathrm{H}_{2}$ line strengths to a simple model in which $\mathrm{H}_{2}$ line strength is invariant while $\mathrm{H}$ I line strength diminishes. This simple model accurately replicates the dependence of the $\mathrm{H}_{2}$-to-H I line ratio on $A_{K}$ for all but the most heavily extincted epoch (for which increasing systematic errors may lead to underestimated errors based on photon statistics alone).

Shocks due to stellar winds and outflows, as well as photoexcitation by high-energy radiation, are both capable of producing $\mathrm{H}_{2}$ emission. Previous studies of Classical T Tauri stars and Class I protostars have identified systems that appear to be templates for each type of $\mathrm{H}_{2}$ excitation mechanism, as well as numerous systems where the excitation mechanism remains ambiguous (Beck et al. 2008; Greene et al. 2010). From their reported 2010 July 14 spectrum, Covey et al. were able to 


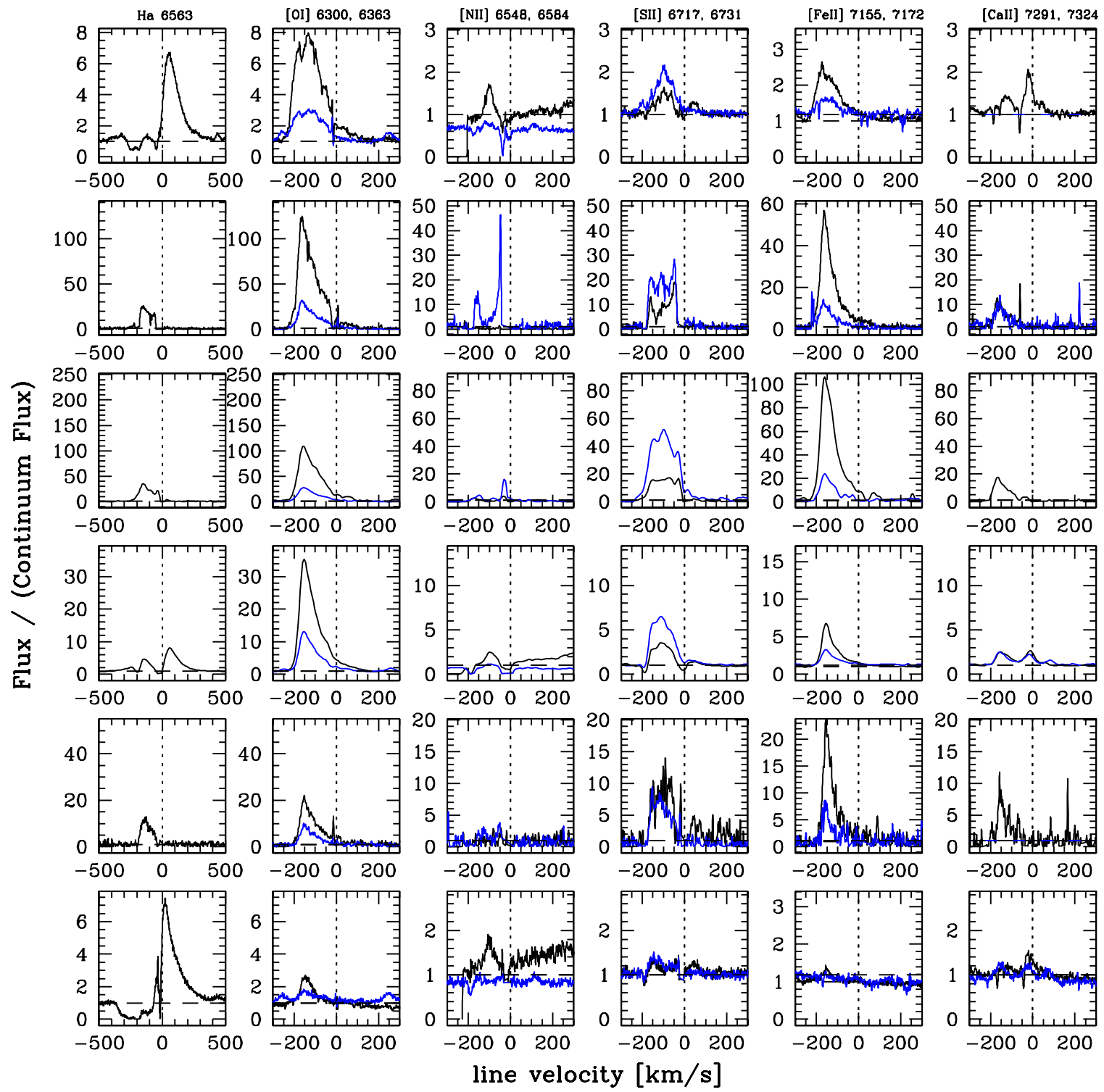

Figure 16. Comparison of normalized optical $\mathrm{H} \alpha$ and forbidden line profiles as identified above the top row. The velocity range along the abscissa is $-/+500 \mathrm{~km} \mathrm{~s}^{-1}$ in the $\mathrm{H} \alpha$ panels and $-/+300 \mathrm{~km} \mathrm{~s}^{-1}$ for all other lines. Rows from top to bottom show data taken on 2010 December 13 (ordinate axis scaled by 1.05 ), 2011 June 15 (scale 18), 2011 June 28 (scale 32), 2011 December 9 (scale 5), 2012 January 6 (scale 7), and 2012 August 7 (scale 1). The ordinate range changes for the different dates (rows) by the scale factors given above, set according to the strength of the strongest emission line. However, the relative scaling among panels along each row is the same, so that comparison of line ratios among dates (rows) is meaningful; for example, [O I] to [Fe II] is high when the source is bright in 2010 December and 2011 December, but low in 2011 June and 2012 January when the source is faint. Black and blue lines indicate, respectively, the first and second wavelengths as labeled at the top. Balmer line emission is visible over the same range of blueshifted velocities as the forbidden lines during faint photometric states, but there is an additional redshifted emission component during bright photometric states. Variation is seen in the ratio of the [O I] 6300 and 6363 lines and the [S II] 6717 and 6731 lines; for reference, the ratios are roughly as expected for optically thin emission in the top row.

(A color version of this figure is available in the online journal.)

measure confidently only the $2.12 \mu \mathrm{m} \mathrm{H}_{2}$ line, as well as state a tentative detection of the $2.24 \mu \mathrm{m} \mathrm{H}_{2}$ line. From the ratio of these two lines, it was concluded that shock excitation was the most likely mechanism for exciting PTF 10nvg's $\mathrm{H}_{2}$ emission, but noted that this conclusion remained uncertain. The 2011 July 15 spectrum, obtained when PTF 10nvg was near a photometric minimum, is by contrast nearly featureless save for a number of well detected $\mathrm{H}_{2}$ and [Fe II] emission features. We present line strength measurements in Table 6 . for nine $K$-band $\mathrm{H}_{2}$ emission lines. For comparison we also tabulate the line strengths expected for different physical emission mechanisms: first models of J- and C-type shocks in stellar outflows (Smith 1995) and models of UV and/or X-ray photoexcitation in the presence of circumstellar dust (Nomura et al. 2007) and, second, empirically measured values for spatially extended $\mathrm{H}_{2}$ emission near RW Aur (Beck et al. 2008) and for a knot in the HH 54 outflow (Giannini et al. 2006). Finally, Table 6 also summarizes the observed and predicted values for specific line ratios identified by Beck et al. (2008) as particularly useful for disentangling the underlying excitation mechanism for $\mathrm{H}_{2}$ emission.

The PTF 10nvg line ratios are extremely similar to those measured by Beck et al. (2008) using adaptive optics imaging that spatially resolves the circumstellar $\mathrm{H}_{2}$ emission near RW Aur. The line strengths measured by Giannini et al. (2006) from Knot B of HH54 are also in reasonable agreement with our observations of PTF 10nvg. This similarity is consistent 


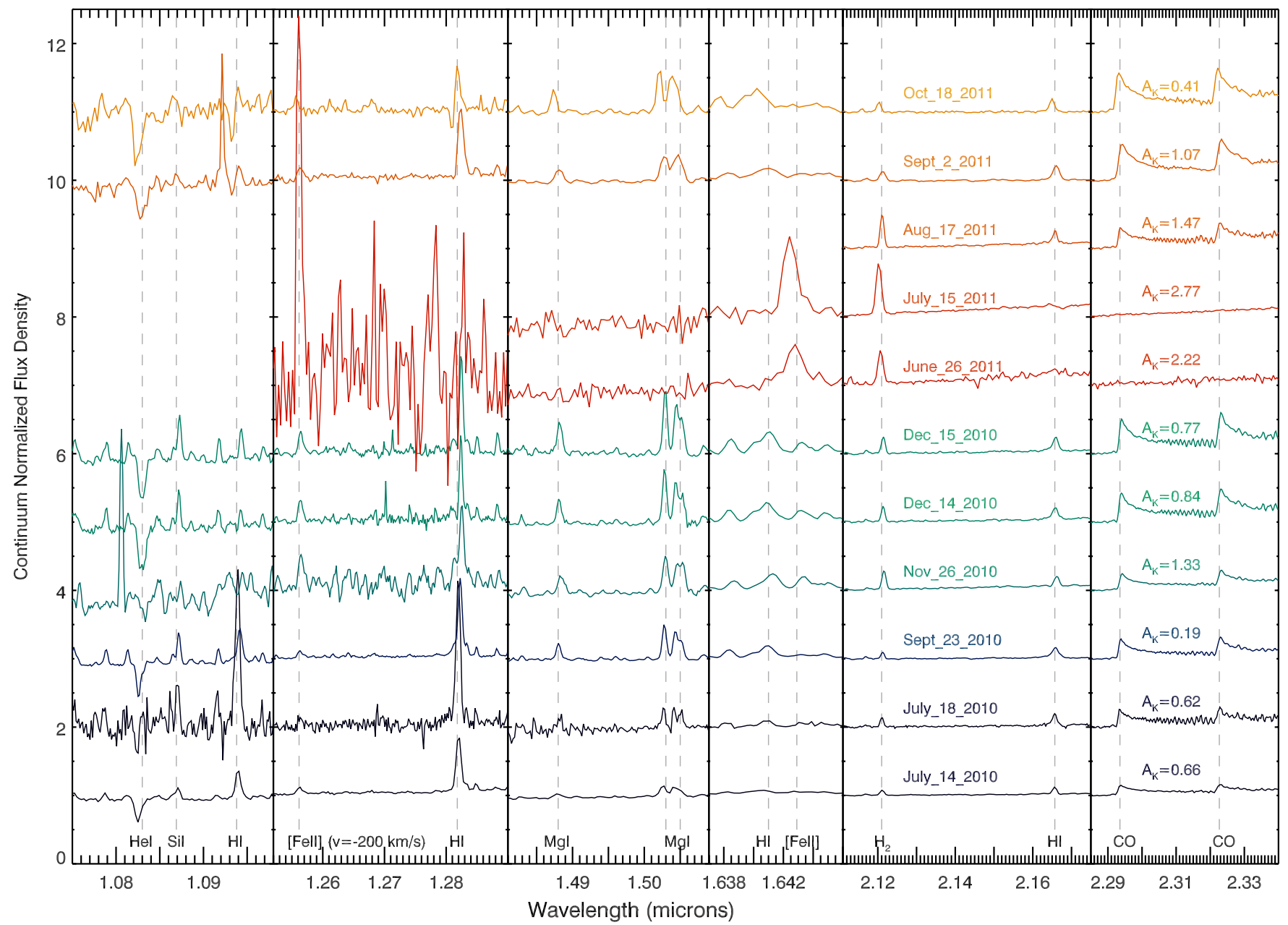

Figure 17. Time-series near-infrared spectra, isolating representative permitted and forbidden transitions. Extinction estimates based on the photometry are indicated in the right panel. Emission lines that are thought to trace hot, dense circumstellar material (i.e., $\mathrm{H} \mathrm{I}, \mathrm{CO}, \mathrm{Mg}$ I) are weaker in higher extinction states whereas lines thought to trace shocks $\left(\mathrm{H}_{2}\right)$ or photoexcitation ([Fe II]) in lower-density circumstellar material are stronger. Absolute line flux measurements reveal that the lower-density tracers maintained a near-constant brightness, suggesting that they are formed exterior to the extincted region, while the lines tracing hotter, denser material faded along with PTF 10nvg's continuum, suggesting that they are formed near the extincted photosphere or inner disk regions.

(A color version of this figure is available in the online journal.)

Table 6

PTF 10nvg $\mathrm{H}_{2}$ Line Fluxes

\begin{tabular}{|c|c|c|c|c|c|c|c|c|c|c|}
\hline Line ID & PTF 10nvg & $\begin{array}{c}\text { B08 RW Aur } \\
\text { (spatially extended) }\end{array}$ & S95 C Shock & S95 J Shock & G06 HH54 & $\begin{array}{l}\text { N07 X-ray } \\
(10 \mu \mathrm{m})\end{array}$ & $\begin{array}{l}\text { N07 UV } \\
(10 \mu \mathrm{m})\end{array}$ & $\begin{array}{c}\text { N07 X+UV } \\
\quad(10 \mu \mathrm{m})\end{array}$ & $\begin{array}{l}\text { N07 X+UV } \\
(1 \mathrm{~mm})\end{array}$ & $\begin{array}{c}\text { N07 X+UV } \\
(10 \mathrm{~cm})\end{array}$ \\
\hline $1.958 \mu \mathrm{m}[1-0 \mathrm{~S}(3)]$ & 1.8 & $\ldots$ & 2.68 & 0.0341 & 15.4 & 1.326 & 8.096 & 14.475 & 1.472 & 0.079 \\
\hline $2.034 \mu \mathrm{m}[1-0 \mathrm{~S}(2)]$ & 0.75 & 0.25 & 1.06 & 0.011 & 4.2 & 0.533 & 3.857 & 6.477 & 0.719 & 0.041 \\
\hline $2.123 \mu \mathrm{m}[1-0 \mathrm{~S}(1)]$ & 2.045 & 1.0 & 3.04 & 0.0266 & 9.59 & 1.565 & 12.968 & 20.725 & 2.526 & 0.142 \\
\hline $2.154 \mu \mathrm{m}[2-1 \mathrm{~S}(2)]$ & 0.041 & $<0.09$ & 0.0539 & 0.0027 & 0.51 & $\ldots$ & $\ldots$ & $\ldots$ & $\ldots$ & $\ldots$ \\
\hline $2.222 \mu \mathrm{m}[1-0 \mathrm{~S}(0)]$ & 0.476 & 0.17 & 0.679 & 0.00526 & 2.58 & 0.357 & 3.256 & 5.023 & 0.667 & 0.04 \\
\hline $2.413 \mu \mathrm{m}[1-0 \mathrm{Q}(2)]$ & 0.557 & 0.25 & 0.749 & 0.0058 & 4.5 & 0.395 & 3.604 & 5.56 & 0.748 & 0.066 \\
\hline $2.248 / 2.123$ & 0.10 & 0.11 & 0.05 & 0.24 & 0.10 & 0.06 & 0.02 & 0.03 & 0.03 & 0.15 \\
\hline $2.034 / 2.222$ & 1.58 & 1.52 & 1.56 & 2.09 & 1.63 & 1.49 & 1.18 & 1.29 & 1.08 & 1.03 \\
\hline $2.248 / 2.073$ & 1.07 & 1.09 & 1.09 & 0.73 & 0.61 & $\ldots$ & $\ldots$ & $\ldots$ & $\ldots$ & $\cdots$ \\
\hline $2.123 / 2.407$ & 1.25 & 1.38 & 1.30 & 1.59 & 0.88 & 1.23 & 1.05 & 1.12 & 0.93 & 0.40 \\
\hline
\end{tabular}

with the hypothesis that the origin of PTF 10nvg's $\mathrm{H}_{2}$ emission is a region that is well separated from the central protostellar source. Models that reproduce the line ratios observed for PTF 10nvg and RW Aur include those calculated by Smith (1995) for a $2000 \mathrm{~K}$ C-type shock and the models produced by Nomura et al. (2007) for pure X-ray photoexcitation.

A final clue to the origin of PTF 10nvg's $\mathrm{H}_{2}$ emission is provided by the kinematics of the lines, which are uniformly 

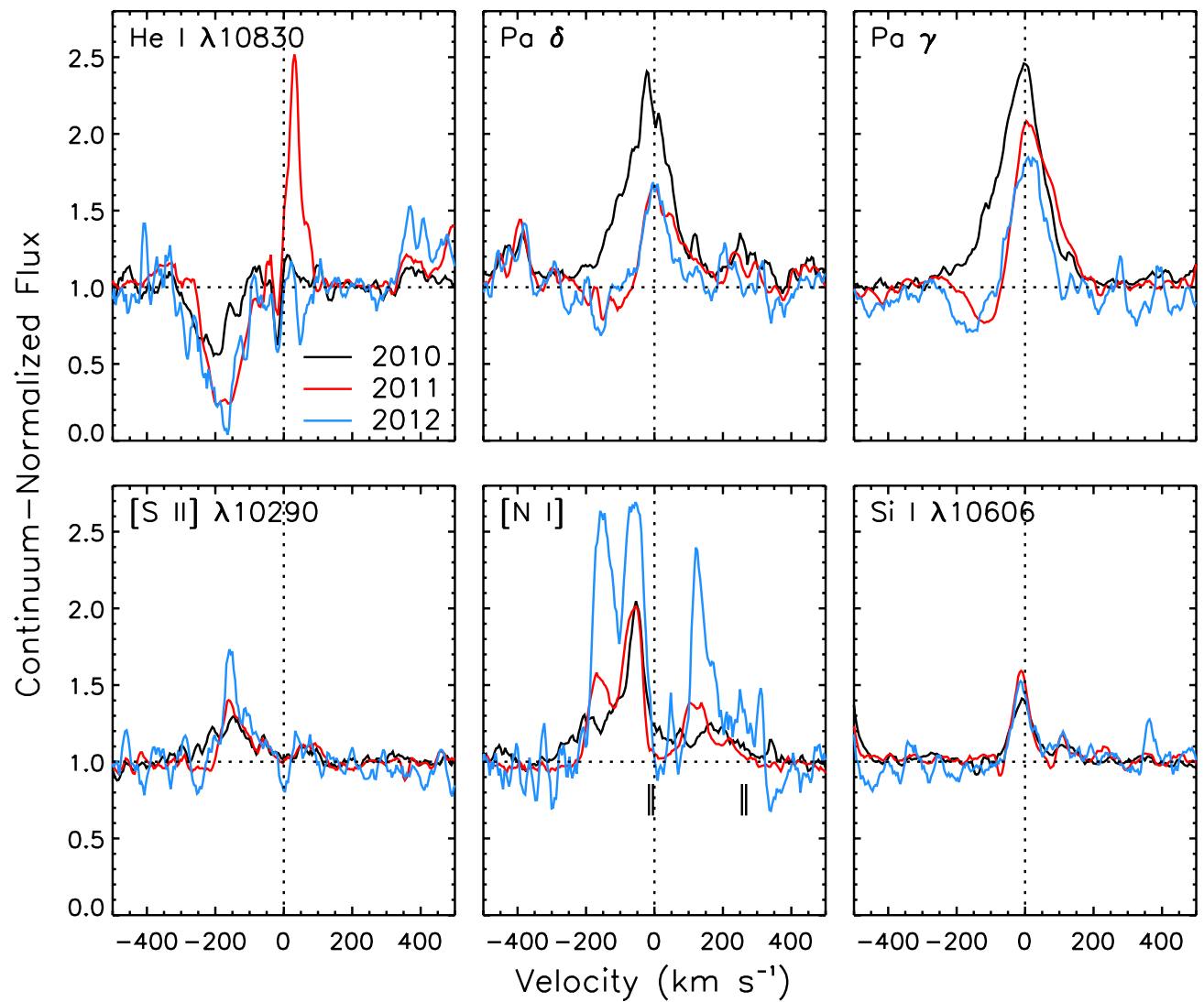

Figure 18. Line profiles in the $1 \mu \mathrm{m}$ region as they appeared in 2010 July (black), 2011 September (red), and 2012 May (blue). Top row shows an He I and two H I lines. Bottom row shows metallic forbidden lines (two [N I] doublets as marked near $1.0401 \mu \mathrm{m}$ and an [S II] line at $1.029 \mu \mathrm{m}$ ) and a permitted line (Si I $1.061 \mu \mathrm{m}$ ). As in the high-dispersion optical spectra, the permitted emission is centered at zero velocity while the forbidden emission is blueshifted along with the wind-indicative He I $1.083 \mu \mathrm{m}$ absorption. Variability over the time series is apparent, with the forbidden lines stronger relative to the continuum during epochs of higher extinction and the $\mathrm{HI}$ lines strong at lower extinction; however, little variability is apparent in the permitted metallic lines.

(A color version of this figure is available in the online journal.)
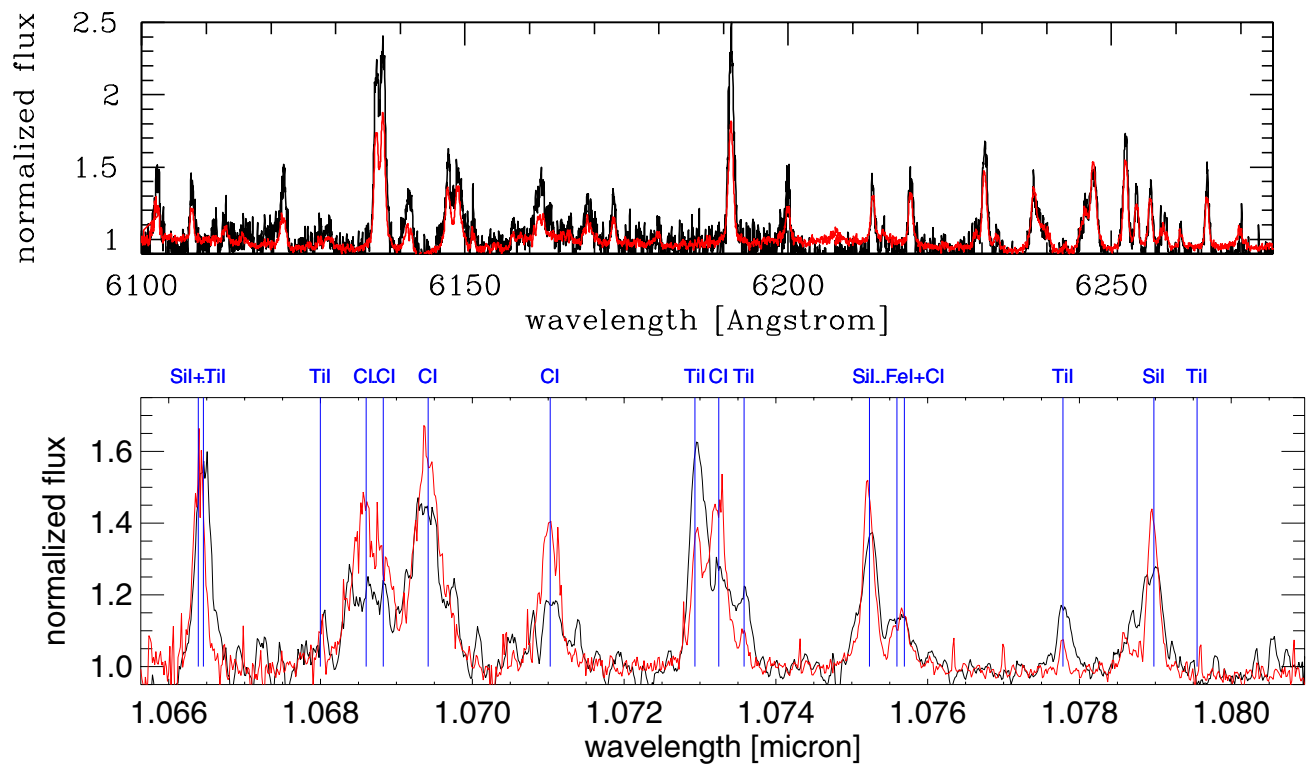

Figure 19. Portions of our high-dispersion spectra for PTF 10nvg (in black) compared to the well-known emission line dominated object V1331 Cyg (in red). There is a clear spectral similarity between these two young stars. Top panel: Keck/HIRES data in the red optical spectral region, showing PTF $10 \mathrm{nvg}$ in a bright state in 2010 December. Bottom panel: Keck/NIRSPEC data in the $Y$ band, showing PTF 10nvg on 2010 July 16, near peak outburst. Blue lines identify various permitted metallic emission lines, which are similar in the two stars. H I, He I, and forbidden metallic lines (located in other spectral regions) are also present in both stars but exhibit different kinematic signatures.

(A color version of this figure is available in the online journal.) 


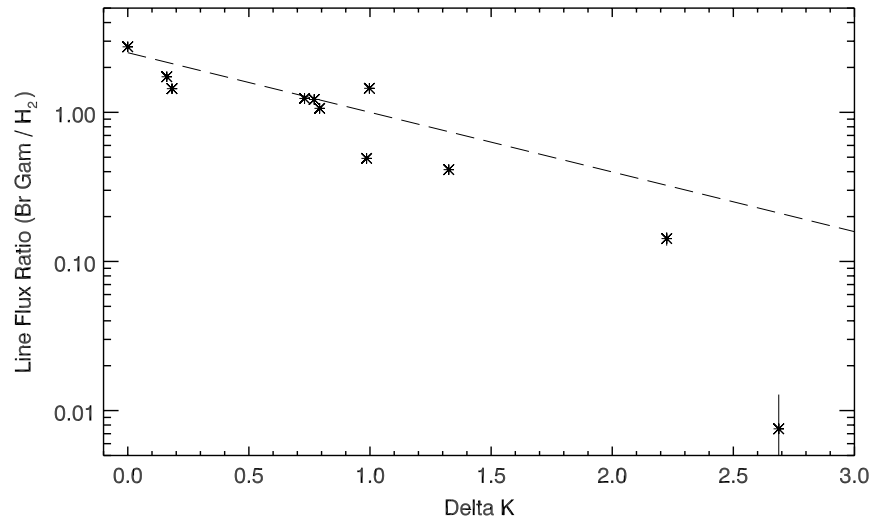

Figure 20. $\mathrm{H}_{\mathrm{I}} 2.16 \mu \mathrm{m}$ to $\mathrm{H}_{2} 2.12 \mu \mathrm{m}$ line ratio measured from moderate resolution near-infrared spectra (asterisks) as a function of PTF 10nvg's $K_{s}$ magnitude relative to peak brightness. Error bars for the ratio measurements were established by adding synthetic noise to the spectrum then re-measuring the line ratios. Overplotted for comparison as a dashed line are the predictions of a simple model in which the $\mathrm{H}_{2}$ line flux is constant with time, while the H I $2.16 \mu \mathrm{m}$ line flux diminishes as expected if the differential $K_{s}$ magnitude is due entirely to extinction (i.e., $\mathrm{HI}=\mathrm{HI}_{o} \times 10^{-0.4 \times \Delta K_{s}}$ ). The anomalous point at high extinction corresponds to the 2011 July 15 epoch where the Brackett gamma line strength is very weak, and therefore may be more susceptible to systematic errors introduced by miscancellation of the $\mathrm{H}$ i profile of the telluric standard. Except for this anomalous point, the observed line ratios agree quite well with the quantitative predictions of the extinction variation model.

blueshifted by $\sim 200 \mathrm{~km} \mathrm{~s}^{-1}$ with respect to the rest velocity of the North America Nebula as well as the observed velocities of PTF 10nvg's permitted atomic features. This kinematic profile strongly suggests that PTF 10 nvg's $\mathrm{H}_{2}$ emission originates from within a stellar jet. Interestingly, isolating the emission region location does not completely resolve the ambiguity between $\mathrm{X}$-ray and shock heating as potential excitation mechanisms, as Chandra observations of the HH 154 protostellar jet revealed that shock regions can generate X-rays in situ in emission regions with expansion velocities of $\sim 500 \mathrm{~km} \mathrm{~s}^{-1}$. Figure 17 demonstrates the behavior of $\mathrm{H}_{2}$ (second panel from right) relative to [Fe II] (third and fifth panels from right) which vary in a correlated manner over the time series, and relative to $\mathrm{CO}$ (rightmost panel) and various permitted atomic lines (all panels) where there is anti-correlated behavior.

In summary, multiple lines of evidence support the conclusion that PTF 10nvg's $\mathrm{H}_{2}$ emission likely arises from a spatially extended outflow. First, the $\mathrm{H}_{2}$ emission strength is insensitive to the variable extinction that apparently occults the innermost disk and accretion region-where the near-infrared continuum and the other molecular (see above) emission as well as the permitted atomic (see below) emission lines form. Second, there is similarity in $\mathrm{H}_{2}$ line strengths to RW Aur's spatially extended emission and to models of excitation due to mechanical shocks and X-ray photoexcitation, both of which have been associated with other protostellar jets.

\subsubsection{Permitted Atomic Emission}

Over most of the time series, the strongest emission lines in the optical spectra of PTF 10nvg are those of $\mathrm{H} \alpha$ and $\mathrm{Ca}$ IItypical situation for young stars. As discussed below, during fainter photometric stages, however, the forbidden lines became stronger than these atomic lines. The $\mathrm{H} \alpha$ and $\mathrm{Ca}$ II line strengths vary both absolutely and relative to the red optical continuum (top and bottom panels of Figure 10).

The Ca II triplet emission varied between -15 and $-25 \AA$ but became much weaker in 2011 June, when the continuum faded.
For classical T Tauri stars in Taurus, the line strengths among the three lines of the Ca II triplet are typically $1.20 \pm 0.19$ for the $8542 \AA$ to $8498 \AA$ ratio and $1.28 \pm 0.13$ for the $8542 \AA$ to $8662 \AA$ ratio (based on analysis of our own collection of optical spectra for representative samples). In PTF 10nvg, however, the $8498 \AA$ line is particularly strong; the $8542 \AA$ to $8498 \AA$ ratio has a mean and dispersion of $0.93 \pm 0.15$ over the time series (considering only spectra for which the lines are measurable), while the $8542 \AA$ to $8662 \AA$ ratio is consistent with expectations at $1.14 \pm 0.18$. Although highly unusual, these observed line ratios for PTF 10nvg are not unique. Hamann \& Persson (1989) found for V645 Cyg ratios among the triplet lines of 0.85 for $8542 \AA$ to $8498 \AA$ and 1.1 for $8542 \AA$ to $8662 \AA$, similar to those in PTF 10nvg. Notably the line ratios are far from their expected ratio of 1:9:5 for optically thin emission, which is typically the case for young accreting stars. Kwan \& Fischer (2011) find that the physical conditions for producing strong Ca II triplet emission include relatively high density $n_{\mathrm{H}} \sim 10^{12} \mathrm{~cm}^{-3}$ and low temperature $T<7500 \mathrm{~K}$, with the line formation taking place in the accretion flow.

As illustrated in the high-dispersion profiles of Figures 15, the Ca II triplet lines all peak to the red of zero velocity. The velocity profiles are similar between the 2010 December and 2011 December spectra (when atomic emission was also prominent) although the 2011 spectra are even narrower on the blueshifted side. There are indications, however, at both epochs of differences among the three lines of the triplet. The $8498 \AA$ line is the broadest of the three, with FWHM $\approx 200 \mathrm{~km} \mathrm{~s}^{-1}$, has an approximately symmetric profile, and exhibits no evidence for sub-continuum absorption. The $8542 \AA$ and $8662 \AA$ lines, by contrast, are somewhat narrower and asymmetric (narrower on the blue side than the red side), with sub-continuum "dips" in their blueshifted components that change between the two highdispersion spectra and indicate wind absorption. The 8542 and $8662 \AA$ structure is similar to that seen in other strong outflow sources such as V1515 Cyg and V1057 Cyg. On its red side, the $8498 \AA$ line traces well the $8662 \AA$ profile but the $8542 \AA$ line is broader; on the blue side, though, it is the 8542 and $8662 \AA$ profiles that are quite similar. While these three lines share the $2 P \rightarrow 2 D$ transition, they differ in their spin with the 8542 and $8662 \AA$ electrons changing spin between the $4 p$ and $3 d$ energy levels, and the $8498 \AA$ electrons retaining the same spin, which is perhaps a clue to the apparent differences in line optical depth.

In $\mathrm{H} \alpha$, the emission line equivalent widths varied at low dispersion between -20 and $-60 \AA$ during brighter photometric states but became quite large (many hundreds of $\AA$ ) as the source continuum level faded during late June through August of 2011. The strength and profile behavior as discerned from our collection of low-resolution spectra is illustrated in Figure 13 while the high-dispersion behavior is illustrated in Figure 16.

The low-resolution spectra from 2010 July, August, and September all show a redshifted emission shoulder in $\mathrm{H} \alpha$, with the first July spectrum exhibiting a clear blueshifted absorption component, which is then ambiguous in the August and September data. The line profile as seen in the high-dispersion 2010 December spectrum shows more clearly both the redshifted emission component and a two-component structure to the blueshifted absorption, similar to the structure seen in the Ca II lines described above. ${ }^{16}$ In $\mathrm{H} \beta$, the line structure has the

\footnotetext{
16 Based on optical spectra taken on 2010 September 5 and November 25, Aspin (2011) claim that the blueshifted $\mathrm{H} \alpha$ absorption reported by Covey et al. had disappeared. Our data show that the absorption weakened in the low-dispersion data but it clearly remained present.
} 
same $-250 \mathrm{~km} \mathrm{~s}^{-1}$ absorption component as seen in the $\mathrm{H} \alpha$ line, but a deeper $-125 \mathrm{~km} \mathrm{~s}^{-1}$ absorption component that extends to lower velocities and even to the positive side of the line. During the faint photometric state spectra (two in 2011 June and one in 2012 January), the $\mathrm{H} \alpha$ profile lacks any redshifted emission and only strong blueshifted emission is observed correlating with strong forbidden-line emission at these same velocities (see Figure 16 and the discussion below). The brighter state (2012 August and 2010 December) profiles are also similar. The 2011 December profile is intermediate, exhibiting both strong blueshifted emission and strong redshifted emission components. The blueshifted emission components in $\mathrm{H} \beta$ and $\mathrm{H} \alpha$ are similar and exhibit similar time-series behavior. Specifically, while from 2011 May through to 2011 October, the $\mathrm{H} \alpha$ line is considerably weaker (Figure 10), the even greater dimming of the red continuum produced enhanced line equivalent width during this period (left panel of Figure 13). The 2011 October and November profiles then revert back to the redshifted emission shoulder, as the source brightened.

This time-series behavior suggests that at least some of the blueshifted $\mathrm{H} \alpha$ emission originates in the outflow or outflow shock region, similar to the $\mathrm{H}_{2}$ emission (discussed above) and to the optical/infrared forbidden line emission (discussed below), both of which are similarly blueshifted. The blueshifted velocities for all of these emission tracers at the position of the continuum source are similar to the velocities reported in Section 5.3.4 for the spatially offset blueshifted emission that is observed, and arises from the interaction of the outflow with either ambient material in the local vicinity or itself.

In both the $\mathrm{Ca}$ II and the $\mathrm{HI}$ profiles there is no apparent change over the existing time series in the velocity of the emission line peaks (which, as described above, are slightly to the red in both of these lines) or in the terminal velocity of the blueshifted absorption components. That there is little change in the terminal velocity of the wind means that the launching velocity is not changing, even though there is changing structure in the absorption part of the P Cygni profiles.

Notably, no optical He I $\lambda 5876$ emission is seen, ${ }^{17}$ even though this line is near-ubiquitously detected for classical $\mathrm{T}$ Tauri stars in high-dispersion spectra. For strong emission at $5876 \AA, T \approx 15,000-20,000 \mathrm{~K}$ and $n_{\mathrm{H}}>10^{11}$ would be required (Kwan \& Fischer 2011) and thus a lack of $5876 \AA$ emission while lower energy $10,830 \AA$ emission is observed could indicate an upper limit on temperature and/or density in the emission region.

Other prominent atomic emission lines in the optical include those of O I, Fe I, Fe II, Ca I. Those lines that are visible in the existing high-dispersion spectra (see Figure 15) are generally symmetric and centered at zero velocity, as well as narrow in velocity with FWHM $\sim 45-50 \mathrm{~km} \mathrm{~s}^{-1}$ (e.g., Fe I lines) to $\sim 65-70 \mathrm{~km} \mathrm{~s}^{-1}$ (e.g., Fe II lines). Their origin may be on the stellar surface or in the disk. The O I $\lambda 8446$ line, however, though also centered at zero velocity, is broadened with FWHM $\sim 250 \mathrm{~km} \mathrm{~s}^{-1}$. This width is similar to that exhibited by the $\mathrm{Ca}$ II and $\mathrm{H} \alpha$ lines, and suggests origin in the inflowing and/or outflowing material. In the low-dispersion spectra we measure a ratio of $\mathrm{Ca}$ II $\lambda 8498$ to O I $\lambda 8446$ (for which the time variable extinction effects are not a factor given the small wavelength difference) that does change somewhat over time but notably always exceeds 10. Kwan \& Fischer (2011) find that values

\footnotetext{
17 A narrow $6678 \AA$ emission line might be associated with He I but the absence of the related $5876 \AA$ line suggests that this is more likely Fe I $\lambda 6678$.
}

larger than 7 for this ratio indicate temperatures $T<7500 \mathrm{~K}$ and densities $n_{\mathrm{H}}>10^{12} \mathrm{~cm}^{-3}$ for the emitting gas. Only the most extreme accretors among well-studied Taurus stars have similar Ca II $\lambda 8498:$ O I $\lambda 8446$ line ratios.

Another notable atomic line seen in emission is $\mathrm{Li}$ I $\lambda 6707$. Interpretation of the profile (Figure 15) should take into account that there is a $y^{3} F^{o}-e^{5} P$ term transition of Fe I reported by the NIST Atomic Spectra Database (Ralchenko et al. 2011) at similar wavelength, specifically $6707.43 \AA$. No other lines of this Fe I multiplet are within our spectral range and so the possible identification as such cannot be supported. We note, however, that another Li I line at $6103 \AA$ may also appear weakly in emission in our spectra, possibly supporting the Li I emission scenario. Although this line also has a nearby Fe I potential identification, the $w^{5} D^{o}-5 / 2[7 / 2]$ term transition at $6103.64 \AA$, this is a much higher excitation Fe I line than those found otherwise in the PTF 10nvg spectra.

Redward, as illustrated in Figure 19, the atomic line emission apparent in the high-dispersion $1 \mu \mathrm{m}$ spectra includes zero velocity C I, S I, Si I, Sr II, and Ti I multiplets. There is no Fe I or Fe II apparent. The three epochs of NIRSPEC spectra, which cover dramatically different brightness stages of PTF 10nvg, show little difference in the metallic line emission profiles (e.g., Figure 18) which generally match quite well over a range of line excitation potentials. Changes over time are apparent, however, in the $\mathrm{H}$ I and He I profiles, with both lines exhibiting blueshifted absorption components as discussed above in the context of wind lines. Other prominent near-infrared emission lines seen in the low-dispersion spectra include $\mathrm{Na}$ I, Mg I, and Fe II. As Figure 17 demonstrates, these lines appear to vary in concert with one another, and are anti-correlated with the extinction estimates inferred from PTF 10nvg's near-infrared photometry.

A common practice is to convert infrared hydrogen line fluxes into mass accretion rates on to the central star. Following Muzerolle et al. (1998) and assuming a mass of $0.5 M_{\odot}$ and radius of $3 R_{\odot}$ for the star, we derive accretion rates of $3 \times 10^{-7} M_{\odot} \mathrm{yr}^{-1}$ in the brighter states and $6 \times 10^{-8} M_{\odot} \mathrm{yr}^{-1}$ in the fainter states using $\mathrm{Br} \gamma$ with an upper limit of $5 \times$ $10^{-12} M_{\odot} \mathrm{yr}^{-1}$ when the line was undetected during summer of 2011 . Using $\mathrm{Pa} \beta$, the values are about a factor of four lower. No extinction corrections are applied in these calculations, and thus the numbers are clearly lower limits in the faint state and probably the bright state as well. In addition to the extinction uncertainty, it is not clear that the accretion model on which the line luminosity to accretion rate calibration is based applies to PTF 10nvg.

\subsubsection{Forbidden Atomic Emission}

In young stars, forbidden lines of, e.g., [O I], [N II], [S II], and $[\mathrm{Fe}$ II] in the optical and near-infrared spectra are attributed to few hundred $\mathrm{km} \mathrm{s}^{-1}$ radiative shocks (those that cool by such radiation more quickly than they would by expansion) in outflowing material in jets associated with accretion disks. Unlike the permitted optical/infrared emission lines discussed above, the forbidden emission lines are (almost always) optically thin. They trace plasma over a wide range in density $n_{e} \sim 10^{3}-10^{8} \mathrm{~cm}^{-3}$ in the temperature range $T \sim 5000-20,000 \mathrm{~K}$-for hydrogen ionization fraction $n_{e} / n_{\mathrm{H}} \approx 1 / 10$, which is appropriate for the mostly neutral young star jets.

The moderate temperature, low density forbidden lines are formed closest to the shock, with temperature decreasing and density increasing further from the shock in the pre-shock medium. The infrared $\mathrm{H}_{2}$ lines discussed above are generally 
thought to form further away from the shock front than the optical/infrared forbidden lines, probing cooler temperatures in either post-shock gas along the jet axis that has sufficiently cooled, or material perpendicular to the jet axis that is entrained/ stirred by the flow. As with the density and temperature gradients, the ionization fraction is likely not constant along the flow. The implied range of physical parameters in the outflow sampled in spectroscopic observations such as ours that do not spatially resolve (see, however, Section 5.3.4) the cooling region(s) in the outflow zone means that we should consider the forbidden line emission indicative but not diagnostic of jet/ outflow conditions. See Hartigan et al. (2000) for a review and Brugel et al. (1981) for a classic study of forbidden lines in Herbig-Haro objects.

Indeed, the typical [O I], [N II], [S II], and [Fe II] forbidden lines are present in PTF 10nvg spectra, but they display significant variability over time (see Figures 13, 14, and 16). The forbidden profiles are purely blueshifted, with a range of profile shapes. Notable in the spectroscopic time series is the increasing prominence of these and other forbidden lines in 2011 June - as the broadband optical/infrared continuum faded to below detectable levels in our PTF $R$-band photometry. Specifically in the red optical spectra, while the somewhat common forbidden line doublets of [O I] $\lambda \lambda 6300,6363$, and $\left[\mathrm{S}_{\mathrm{II}}\right] \lambda \lambda 6717,6731$ as well as $[\mathrm{Fe} \mathrm{II}] \lambda \lambda 7155,7172$ and [Ca II] $\lambda \lambda 7291,7324$, had been weakly present in the $2010 \mathrm{spec}-$ tra and in the first 2011 (May) spectrum obtained, by 2011 June these lines had significantly increased in strength relative to the continuum. Over just a three week time frame, they along with other somewhat unusual forbidden lines (such as the $\left[\mathrm{CI}_{\mathrm{I}}\right] \lambda \lambda 9824,9850$ doublet, [CrII], and [Ni II]; see Figures 10, 13, and 14) rose in prominence to dominate the optical spectrum. Notably, emission in [N II] is seen only during the very faintest epochs. This increasing prominence of the forbidden lines was accompanied by a weakening of the permitted emission lines, most visibly the Ca II triplet and several Fe II lines that previously were quite strong. This behavior suggests that we are seeing, in the faint photometric states, emission from a Herbig-Haro flow that is not visible when the source is brighter.

We can gain insight into the physical conditions necessary for these forbidden line observations by taking guidance from the material assembled in Dougados et al. (2010) and references therein. For the different forbidden line species we discuss first the line strengths and their variation, and then the line profiles and their variation.

For the [OI] 6300 and $6363 \AA$ doublet, having the highest critical density of the red optical [O I], [N $\mathrm{NI}]$, and [S II] lines, the evolution of the observed line ratio is truly remarkable. Typically observed [OI] $\lambda 6300:\left[\mathrm{O}_{\mathrm{I}}\right] \lambda 6363$ values are in the range 2.5-4:1 with the theoretical line ratio 3:1 based on Einstein $A$ values. Before and after the deep photometric fading episode of 2011, the expected 3:1 ratio for optically thin emission was indeed consistently observed to within $10 \%$ in PTF 10nvg spectra. However, as the continuum flux faded and the forbidden line prominence increased, the [O I] doublet ratio was variable between the typical 3:1 and values as extreme as 1:2 in several of the faint-state 2011 June, July, and August low-dispersion (see Figure 13) as well as high-dispersion (see Figure 16) spectra. The variability in the doublet line ratio suggests dramatic changes in the optical depth of the [O I] lines, from optically thin to a likely optically thick case where $1: 1$ is expected; we cannot, however, explain the observed situation that the $6363 \AA$ line is stronger than the $6300 \AA$ line at some epochs.
We note that Li \& McCray (1992) developed a model for [O I] $\lambda 6300:\left[\mathrm{O}_{\mathrm{I}}\right] \lambda 6363$ doublet emission in SN 1987A, which exhibited a systematically time variable ratio that increased from an optically thick $1: 1$ to the usual optically thin $3: 1$. The model was based on an expanding shell with a small filling factor, i.e., clumpy distribution of [O I], that is not necessarily applicable here given differences in expected geometry between a spherically expanding supernova shell and a more collimated young star outflow, but does suggest that variable line optical depth is a plausible explanation for our observations. Notably, the [O I] changes observed in PTF 10nvg were not gradual and systematic as observed in SN 1987A; rather, they were abrupt with the $6300 \AA: 6363 \AA$ ratios changing literally from night to night, e.g., from 1:1 on 2011 August 1 to $3: 1$ on 2011 August 2 when the data derive from the same instrument, configuration, observers, slit position angle, etc. so the variability cannot be attributed to spatial alignment or other similar observational effects. Plausible explanations for our observations, whether related to optical depth effects or not, need to account not only for the truly peculiar 1:2 line ratio but also the short term variability of the ratio.

At all epochs, including during the photometric fading period when the $\left[\mathrm{O}_{\mathrm{I}}\right]$ ratio is out of the expected $3: 1$ ratio, the much lower critical density [S II] $\lambda \lambda 6717,6731$ doublet notably remained within the expected range for its ratio. Typically observed values in young stars are $0.5-1: 1$, with the theoretical ratio in the high density limit 0.43 . The PTF data are consistent with these values but with some variation over the time series. Most of the data exhibited line ratios close to the high density expectation (indicating $n_{e}>10^{4} \mathrm{~cm}^{-3}$ ) but some epochs show more equal strength doublet lines, indicating lower density gas $\left(n_{e} \sim 10^{3} \mathrm{~cm}^{-3}\right)$.

Notably, neither [O III] 4959/5007 with a similar critical density to the strong [O I] doublet, nor [O II] 3726/3728 with a similar critical density to the strong [S II] doublet, are seen. Although our spectra become somewhat signal-to-noise challenged in the blue, strong line emission comparable to the red line emission would be detectable. The [O III] line absence provides a constraint on the electron temperature of the emitting gas and is, indeed, rarely seen in well-studied $\mathrm{HH}$ objects. The [O II] absence can be taken to confirm the assumption of a low ionization fraction.

The $[\mathrm{N}$ II $] \lambda \lambda 6548,6583$ doublet is of intermediate critical density and has a typically observed ratio of 1-5:1 in young stars with a theoretical ratio of 3 based on Einstein A values. These lines are present in just a few of our low-resolution spectra during the faintest photometric states, but are hard to disentangle from the broad Halpha emission (see Figure 13). They were only weakly visible in our high-dispersion data. We note that $[\mathrm{N} \mathrm{II}] \lambda \lambda 6548,6583$ emission is seen, however, at positions that are spatially offset from the PTF 10nvg point source, as discussed in Section 5.3.4. Some of the weak emission seen in Figure 16 may arise in the extended emission region closest to the trace (see Figure 21).

Considering a higher density diagnostic, the infrared [Fe II] 1.533 and $1.644 \mu \mathrm{m}$ lines, like the optical forbidden lines, are more prominent when the source is faint in the continuum. The ratio of these two lines is density sensitive and indicates $n_{e}>10^{5} \mathrm{~cm}^{-3}$. The 1.257 and $1.644 \mu \mathrm{m}$ line ratio can be used to measure extinction since the lines share the same upper level (see Connelley \& Greene (2010)). For PTF 10nvg the time-series data indicate low extinction $\left(A_{V}<2 \mathrm{mag}\right)$ to the jet region-in contrast to our earlier findings on the large and 

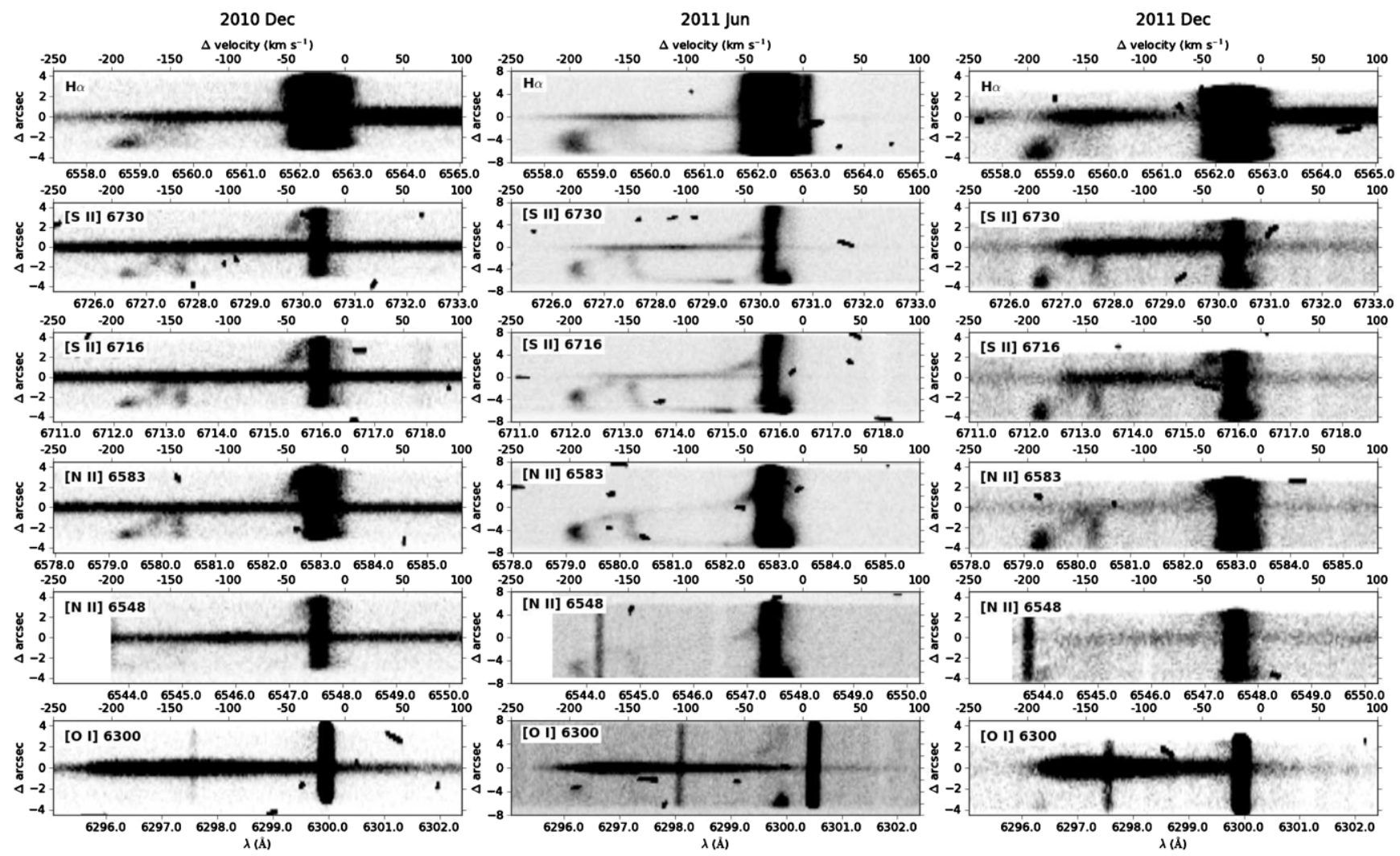

Figure 21. Spatial-spectral diagrams centered around the optical $\mathrm{H} \alpha$, [O I $]$, [N II], and [S II] lines. Units on the ordinate are arcseconds while those on the abscissa are given in both wavelength $(\AA)$ and velocity $\left(\mathrm{km} \mathrm{s}^{-1}\right)$ with each panel showing a $350 \mathrm{~km} \mathrm{~s}^{-1}$ portion of the emitting region. The image intensity scaling is linear with the range set over a range of counts selected for each feature to emphasize the spatially extended emission (not the same in all panels). Cosmic rays have not been removed from the images. "Sky" emission fills the decker vertically at low-velocity locations that depend on heliocentric correction and may not be the same from line to line depending on whether the emission is dominated by telluric vs. nebular background emission. The left and right panels show the HIRES observations from 2010 December (P.A. $=110^{\circ} 8$ ) and 2011 December (P.A. = 102.6), respectively. The middle panel shows data from 2011 July $($ P.A. $=176.0)$ when the decker setting allowed twice the spatial coverage of the other two panels but otherwise exhibits similar structure away from the main trace. Blueshifted emission is seen below the trace at several velocities (roughly $-190,-160,-140$, and $-20 \mathrm{~km} \mathrm{~s}^{-1}$ in all columns plus at -75 and blueward in the middle panel only, with its larger extent in the spatial direction). Blueshifted emission is also seen above the trace at about $-50 \mathrm{~km} \mathrm{~s}^{-1}$.

Table 7

PTF 10nvg [Fe II] Line Fluxes

\begin{tabular}{lcc}
\hline \hline Line ID & PTF 10nvg & G06 HH54 \\
\hline $1.257 \mu \mathrm{m}$ & 4.5 & 8.7 \\
$1.644 \mu \mathrm{m}$ & 5.06 & 8.2 \\
$1.257 / 1.644$ & 0.89 & 1.06 \\
\hline
\end{tabular}

time variable extinction toward to near-infrared continuum. The above and other infrared Fe II lines that are prominent during PTF 10nvg's faintest epochs are tabulated in Table 7 and can be compared in line strength ratio to that observed by Giannini et al. (2006) from Knot B of HH54. The two sets of measurements agree moderately well, providing additional support for the existence of a significant outflow within the PTF 10nvg system. The optical [Fe II] lines probe even higher density material. The measured $7155 \AA, 7452 \AA, 8617 \AA$, and $8892 \AA$ lines of [Fe II] yield ratios that according to Bautista \& Pradhan (1998) indicate gas at densities of $n_{e} \sim 1-5 \times 10^{6} \mathrm{~cm}^{-3}$ in the vicinity of PTF 10nvg. The optical [Fe II] doublet at $7155,7172 \AA$ varies in our time series, strengthening during the 2011 faint epochs but perplexingly failing to re-emerge in the late 2011/early 2012 fade like other forbidden lines. These lines also exhibit a ratio both well above and below its expected 3:1 ratio for optically thin emission. Unlike the time-series behavior of the [O I] doublet ratio, however, the [Fe II] anomalies are not restricted to the faint epochs. There is no correlation of the [Fe II] line ratio variations and the $[\mathrm{O} \mathrm{I}]$ line ratio peculiarities.

High density gas is also probed by [Ni II] and [Ca II] lines. The observed ratio of [Ni II] $7412 \AA$ to $7378 \AA$ in the $0.3-0.5$ range indicates densities $n_{e}>5 \times 10^{7} \mathrm{~cm}^{-3}$ according to Bautista et al. (1996). The observed ratio of Ca II $8542 \AA$ to [Ca II] $7291 \AA$ varies between 8 and 15 during the bright photometric states and is indicative of densities in the range $n_{e} \sim 3-5 \times 10^{8} \mathrm{~cm}^{-3}$. During the faint states this ratio becomes very small as the atomic emission is not detected while the forbidden emission becomes quite strong relative to the continuum.

As illustrated in Figure 18, there is also forbidden line emission in the high-dispersion $1 \mu \mathrm{m}$ spectrum, with a blueshifted set of [ $\left.\mathrm{N}_{\mathrm{I}}\right]$ doublets around $1.040 \mu \mathrm{m}$ (specifically 1.040059, 1.040100 and $1.041002,1.041044 \mu \mathrm{m}$ ) and a blueshifted [S II] series located at 1.029, 1.032, 1.034, $1.037 \mu \mathrm{m}$. They are indicative of hotter temperature gas, in the 30,000-40,000 $\mathrm{K}$ range, than the optical forbidden lines which probe 5000 to $20,000 \mathrm{~K}$ temperatures. Like the optical lines, the near-infrared forbidden line strength appears to correlate with the photometrically derived extinction estimates (Figure 9); specifically, the 2012 spectrum exhibits stronger [N $\left.\mathrm{N}_{\mathrm{I}}\right]$ and [S II] lines than the 2011 and 2010 spectra, both of which were taken when the extinction was about half of the value for the 2012 spectrum. The non-simultaneous optical-infrared spectroscopy in combination 
with the unknown extinction and evidence for large extinction variations over time precludes us from using infrared-optical line pairs of these species to probe either temperature (e.g., from optical $6730 \AA$ versus infrared $1.03 \mu \mathrm{m}$ [S II] at high temperature, or optical $8620 \AA$ versus infrared $1.64 \mu \mathrm{m}[\mathrm{Fe}$ II] at lower temperature) or ionization fraction (e.g., from optical [N II] and infrared $\left.\left[\mathrm{N}_{\mathrm{I}}\right]\right)$. We simply note the presence of these hotter lines in the $1 \mu \mathrm{m}$ region spectra.

In addition to the line strength variations, there were variations in the line profiles of the forbidden emission in PTF 10nvg. These are illustrated by our high-dispersion optical data as shown in Figure 16. In all forbidden lines and at all epochs, the profiles are purely blueshifted with maximum emission velocities of $\sim 250 \mathrm{~km} \mathrm{~s}^{-1}$. At the earliest 2010 December epoch, the [OI] doublet has a broad FWHM and nearly square profile ranging from 0 to $-250 \mathrm{~km} \mathrm{~s}^{-1}$, peaking at about $-130 \mathrm{~km} \mathrm{~s}^{-1}$, whereas at the later epochs the profile becomes skewed with a broad base but increasing flux toward higher velocities, peaking near the terminal velocity, which remains at the same $-250 \mathrm{~km} \mathrm{~s}^{-1}$ terminus. The skewed profile is present in both fainter and brighter continuum states of PTF 10nvg following the initial 2010 December spectrum in which there was more lower velocity emission relative to later times. Similar line profile morphology and behavior over time is seen in the $\left[\mathrm{Fe}_{\mathrm{II}}\right]$ doublet. The [S II] doublet seems to have the opposite trend, however, with a similarly shaped but narrower and slightly lower velocity $\left(-100 \mathrm{~km} \mathrm{~s}^{-1}\right)$ peak relative to the [O I] doublet early on, but becoming more boxy and broad as the [O I] became more skewed. The [N II] doublet appears quite similar to the $[\mathrm{S}$ II] doublet but is much weaker, and essentially undetected in most of our high-dispersion spectra.

The [Ca II] doublet, unlike the above lines, is double-peaked, with a higher velocity component centered at about $-150 \mathrm{~km} \mathrm{~s}^{-1}$ and extending to $-200 \mathrm{~km} \mathrm{~s}^{-1}$ (similar to the [S II] and [N II] doublets), as well as a second, nearly zero velocity but slightly blueshifted peak. This double-peaked profile (see Figure 16) is seen in the 2010 December and 2011 December spectra, both taken near photometric peaks in the light curve. During the fainter photometric states, however, only the blueshifted peak is seen, and presenting the same skew presented by the $[\mathrm{OI}]$ and $[\mathrm{Fe} \mathrm{II}]$ doublets. A similar double-peaked and timeseries behavior is exhibited by the optical [C I] lines. The [Ca II] doublet, unlike other forbidden lines, has a possible origin in photon pumping through the permitted Ca II 8498, 8542, and 8662 lines rather than requiring collisional pumping (though collisions may still be the dominant mechanism in young star jets). The $7324 \AA: 7291 \AA$ line ratio of [Ca II] is consistent with the expected equilibrium value of 0.67 .

In summary, the forbidden emission line strengths in PTF $10 \mathrm{nvg}$ indicate a wide range of gas densities, from $n_{e} \sim$ $10^{3} \mathrm{~cm}^{-3}$ to $n_{e}>10^{8} \mathrm{~cm}^{-3}$. Line strength variations, some line ratio variations, and line profile variations were all seen over the time series.

\subsubsection{Spatially Offset Emission}

As discussed above, the velocity structure along the spectral trace as defined by the (sometimes weak) spectral continuum exhibits blueshifted emission in a large number of forbidden lines as well as in $\mathrm{H} \alpha$, notably during the fainter photometric states of PTF 10nvg's light curve. In addition, many of the HIRES images exhibit blueshifted emission that is spatially offset from the continuum source position. This is illustrated via position-velocity diagrams in the vicinity of $\mathrm{H} \alpha$ and forbidden
[OI] $\lambda 6300,\left[\mathrm{~N}_{\mathrm{II}}\right] \lambda \lambda 6548,6583$, and $\left[\mathrm{S}_{\mathrm{II}}\right] \lambda \lambda 6716,6730$ in Figure 21.

We show the two-dimensional HIRES spectra at three epochs: 2010 December when PTF 10nvg was relatively bright, 2011 June when it was quite faint, and 2011 December when it was again bright. The two December spectra were obtained at similar position angle (P.A.), $110^{\circ} .8$ and $102^{\circ} .6$ for the 2010 and 2011 observations, respectively, with a $7^{\prime \prime}$ long decker, while the 2011 June spectrum was obtained with a $14^{\prime \prime}$ long decker and a P.A. of 176.0. Despite the differing P.A.s between the spectra, there are a few features that are commonly observed at all epochs. These include a bright knot of emission extending $\sim 2-6$ " from the stellar trace at $\sim-190 \mathrm{~km} \mathrm{~s}^{-1}$, which can be seen in all epochs in $\mathrm{H} \alpha$, [S II], and [N II] $\lambda 6583 \AA$ (note that only the 2011 June spectrum had a long enough decker to see the emission 6 " from PTF 10nvg). This same knot is also visible in [N II] $\lambda 6548$ in the two 2011 spectra as well as [O I] $\lambda 6300$ in the 2011 December spectrum. In addition, there is plausible but weak offset emission in $[\mathrm{Ca}$ II $] \lambda 7291$ at this same $-190 \mathrm{~km} \mathrm{~s}^{-1}$ velocity, but in the $7324 \AA$ line of the doublet it is not obviously offset from a sky line that coincidentally appears at a similar velocity; these images are not shown. Fainter knots, apparently extending from the position of the continuum source, can be seen a few arcsec below the trace at $\sim-140$ and $-160 \mathrm{~km} \mathrm{~s}^{-1}$. These features are present in all three epochs and are most easily seen in [S II]. The final knot that can be seen in all epochs is $\sim 2^{\prime \prime}$ above the stellar trace at $\sim-50 \mathrm{~km} \mathrm{~s}^{-1}$, and it too is most readily identifiable in $[\mathrm{S}$ II].

Interestingly, in the 2011 June spectrum there is virtually no $[\mathrm{N}$ II] emission from the PTF 10nvg continuum position, and for $\lambda 6583$ in particular, it can be seen that each of the aforementioned knots are connected spatially. This structure is clear evidence that PTF 10nvg is driving a jet with a terminal shock ending $\sim 4^{\prime \prime}$ from the star and maximum velocity $\sim 200 \mathrm{~km} \mathrm{~s}^{-1}$. The appearance of these features at all epochs, regardless of the P.A., suggests that PTF 10nvg is driving a wide-angle outflow.

There are additional spatially offset features which are not observed in each epoch. The most prominent is a knot seen in the 2011 June spectrum at $\sim-20 \mathrm{~km} \mathrm{~s}^{-1}$ located $\sim 4^{\prime \prime}$ below the trace of PTF 10nvg and extending to the edge of the decker $\sim 7^{\prime \prime}$ from PTF 10nvg. This feature is most easily identified in [O I] since it is blended with diffuse nebular emission in all the other panels. This feature is too far from PTF 10nvg to be detected in the 2010 December spectrum as the decker did not extend $4^{\prime \prime}$ below the PTF 10nvg continuum, while the same emission feature may be present in the 2011 December spectrum, though blending with nebular emission (or the [O I] night sky line) make a positive identification challenging. Finally, the last prominent feature, which is seen only in the 2011 June observation, is a point-source-like trace seen $\sim 7^{\prime \prime}$ below PTF 10nvg. This trace is seen in $\mathrm{H} \alpha$, $[\mathrm{S} \mathrm{II}]$, and [ $\mathrm{NII}] \lambda 6583$. Optical imaging does not reveal any point sources within $\sim 8^{\prime \prime}$ of PTF 10nvg and so the origin of this emission is currently unclear. High angular resolution narrow-band imaging of the field around PTF 10nvg should elucidate if this feature is from another star or somehow related to PTF 10nvg itself.

No spatially offset emission is apparent in the NIRSPEC $1 \mu \mathrm{m}$ high-dispersion spectral imaging data, e.g., in [N I] or [S II], or in the $1.083 \mu \mathrm{m} \mathrm{He}$ I line. For these observations the slit length was $12^{\prime \prime}$ and thus able to sample the relevant spatial range where offset emission is seen in optical forbidden lines, as discussed above. 


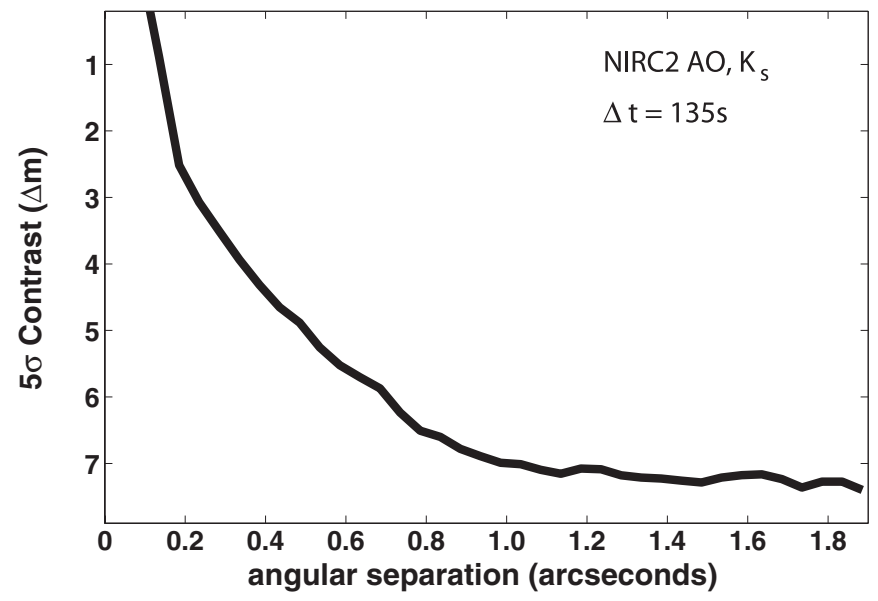

Figure 22. Contrast vs. angular separation curve for the field centered on PTF $10 \mathrm{nvg}$. A maximum contrast of $7.5 \mathrm{mag}$ is achieved in the $K_{s}$ band beyond $1^{\prime \prime}$.0 separation, corresponding to 520-600 AU depending on the adopted source distance. Detailed image analysis reveals that no stellar companion candidates are detected above these limits though the observations cannot rule out very low mass stellar or any substellar companions.

\section{CONSTRAINTS ON MULTIPLICITY}

Neither the raw nor the reduced and co-added adaptive optics imaging frames from Keck/NIRC2 show evidence for point source companions within several arcseconds of PTF 10nvg. Figure 22 shows the sensitivity of the observations to off-axis sources. The diffraction-limited data rule out the presence of companions down to a level of $\Delta K_{s}=$ $3.2,5.0,7.0$, and $7.4 \mathrm{mag}$ at $0^{\prime \prime} .25,0^{\prime \prime} .50,1^{\prime \prime} .0$, and $2^{\prime \prime} .0(5 \sigma)$, respectively. For comparison, the typical FWHM of our seeinglimited PTF observations in the $R$ band and the beam size of our CARMA data at $2.7 \mathrm{~mm}$ are both about $2^{\prime \prime}$.

With a primary source brightness of $K \approx 8.5 \mathrm{mag}$ at the time of the AO observations, the achieved contrast limits correspond to a lack of companions brighter than $K \approx 16$ mag outside of the optical and millimeter PSF. At closer separations, the flux limits can be translated into upper mass limits to any companions of $1.14,0.66$, and $0.30 M_{\odot}$ at separations of 130,260 , and $520 \mathrm{AU}$. These numbers use the isochrones of Girardi et al. (2002) and assume an age of $1 \mathrm{Myr}$, a distance of $520 \mathrm{pc}$, but do not include a foreground extinction estimate since this is unknown (but would raise the mass limits). The companion upper mass limit sensitivity is not particularly deep despite the large contrast achieved with Keck/NIRC2 since the $2 \mu \mathrm{m}$ brightness of PTF $10 \mathrm{nvg}$ is dominated by circumstellar excess rather than pure photosphere.

For reference, companions to several well-known FU Oritype stars do reside within this separation and contrast range: at $196 \mathrm{AU}$ and $\Delta K_{s}=4.1 \mathrm{mag}$ for FU Ori (Wang et al. 2004; Pueyo et al. 2012) and at $115 \mathrm{AU}$ and $\Delta K_{s}=1.2 \mathrm{mag}$ for Z CMa (Koresko et al. 1991; Hinkley et al. 2012). Although the companions have been claimed to have some relevance to the outbursting behavior of FU Ori stars, the ability for such wide companions to instigate the inner disk instability is limited. Companions are also invoked in several examples of presumed disk-eclipsing young stars that, like PTF 10nvg, have long periods. Specifically, KH15D at 48 days (Hamilton et al. 2012), WL4 at 131 days (Plavchan et al. 2008), and YLW16A at 92 days (Plavchan et al. 2010) have extinction-like flux dips between a few tenths and several magnitudes in depth. Several of these stars have companions in the 25-50 AU region that are hypothesized to induce warps in the disks. Our existing AO observations are not sensitive to such close binaries.

\section{DISCUSSION AND INTERPRETATION}

\subsection{The Nature of the Large-amplitude Variable PTF 10nvg}

In this section, we provide broader context for the observations of PTF 10nvg that we have described above, namely, (1) the large-amplitude optical/near-infrared magnitude and color variations that are broadly consistent with expectations from extinction variations; (2) the semi-periodic nature of the multiband light curves; (3) the respective correlation/anti-correlation of permitted/forbidden atomic spectral line emission strength with continuum brightness; (4) the rare detection of molecular $\mathrm{TiO} / \mathrm{VO}$ emission at optical wavelengths, with corresponding molecular $\mathrm{CO}, \mathrm{H}_{2} \mathrm{O}$, and $\mathrm{VO}$ emission in the near-infrared; (5) the exhibition of strong $\mathrm{P}$ Cygni line profiles in several atomic species that are sensitive to winds and outflows; (6) the lack of evidence for stellar multiplicity; (7) the Class I-type SED; and (8) the spatially extended molecular outflow.

Before the 2010 brightening that was detected by both Itagaki et al. and Covey et al., the source discussed here as PTF 10nvg was known as IRAS 20496+4354 but had not been well studied. Although there was no previously reported nearinfrared or optical photometry for the object, Aspin (2011) and we in this paper have demonstrated through investigation of archival images that the source has been sporadically visible in several past observations. The recent photometric behavior included brightening on a several month timescale followed by an overall fade that has been punctuated by semi-periodic spikes in brightness over the past several years.

Both previous to and subsequent to its 2010 brightening, the source can be characterized as a Class I (disk-plus-envelope) young stellar object. Spectroscopically, PTF 10nvg is highly unusual for a Class I, or any other class, young star. In addition to the rare $\mathrm{TiO} / \mathrm{VO}$ emission and more common $\mathrm{CO}$ emission as well as permitted atomic emission during bright photometric states, in its faint photometric state the source is dominated by forbidden emission lines, some of which are commonly seen in young stars but others of which are rarely seen.

One interpretation is that the object could be in its initial envelope-clearing stage such that the embedded optical/infrared point source is in the process of becoming revealed. The consistency of both the photometric and spectroscopic behavior with variations in line-of-sight extinction supports this interpretation. Further, the molecular emission could be the result of heating and cooling at high latitudes in the outflow cavity as stellar photons first reach these areas of the circumstellar environment. As an analogy, classical novae, which are also driven by accretion (on to a white dwarf of material from an unseen companion) frequently show "dust dips" in their outbursting light curves with several episodes of fading and re-brightening occurring on tens to hundreds of days timescales, similar to the situation we have found for PTF 10nvg. Another interpretation is that, instead of repeated geometric clearing of the line of sight to the central star and subsequent obscuration of this line by dust, the source is surrounded by dust that is being in situ reformed in the nearby circumstellar environment and then either destroyed or accelerated away though wind/outflow. In this scenario, an analogy to the deep occulting phenomenon of R Coronae Borealis stars (Clayton 2012) could be appropriate. The very large extinction variations, $\Delta A_{V}$ up to 30 , and the detected periodicity may be a challenge for this hypothesis, however. The quasi-periodicity 
of the dust obscuration events in PTF 10nvg strongly suggest that the appropriate geometry is one of dense material along our line of sight to the optical/near-infrared continuum, that is in orbit about the central star, and located approximately $0.7\left(M_{*} / M_{\odot}\right)^{1 / 3} \mathrm{AU}$ from it.

The periodicity and the extinction-dominated nature of the 2011 and 2012 light curves do not, however, extrapolate back to the 2010 (optical) data in a satisfactory manner, though the limited 2009 data do seem to phase well with the derived period. During 2010 the source was much brighter than over the next two years, and in fall of 2012 the light curve again reached a similar level of brightness (in a departure from the previously observed quasi-periodicity). These unusually bright periods perhaps indicate that at least some of behavior is driven by accretion-related "outburst" events. The recent outburst hypothesis may be further supported by the lack of detection in previous infrared imaging surveys, notably 2MASS, but detection at not fainter than $K_{s} \approx 11$ mag over the past three years. Brightening in the mid-infrared as well, specifically during 2010, suggests a bolometric luminosity increase; however, the scale of the mid-infrared brightening could plausibly be due to the inferred extinction changes. Dramatic photometric and spectroscopic changes in young stars are often classified as outbursts and associated with large scale (one or more orders of magnitude) increases in the accretion rate of material from the disk on to the central star. Below we pursue the interpretation that the body of evidence in hand for PTF 10nvg is indeed indicative of an outbursting young star, though mediated by semi-periodic dust obscuration events.

Spectroscopically, PTF 10nvg bears strong resemblance during its brighter states in the atomic emission line pattern to the well-known extreme emission line object V1331 Cyg. The accompanying forbidden line emission in PTF 10nvg is explained if the enhanced accretion episode of 2010 also drove an enhanced outflow, perhaps with outflowing material reaching a shock-enabling surface approximately one year post-outburst, when forbidden line prominence increased significantly. However, given that we see a richer forbidden spectrum when the source is in a faint state, and given the evidence for large variations in extinction toward the central continuum source, another interpretation is that it is only when the continuum (arising from the photosphere, accretion shock, and inner disk regions) is suppressed that we are able to see a much fainter, spatially distinct, but ever-present jet region. This scenario requires an inclined disk to do the obscuring, but not too inclined given the $>200 \mathrm{~km} \mathrm{~s}^{-1}$ line widths of the [O I], [S II], and [Fe II] lines. The situation would be similar to that advocated by White \& Hillenbrand (2004): there is an orientation effect such that the spatially extended forbidden line emission is more readily observed in systems without a direct view of the central continuum source. This explanation was preferred to invoking differences, or in our case variations in, the mass outflow rate itself for explaining stronger forbidden line emission. We note that Appenzeller et al. (2005) also found enhanced line-to-continuum ratios for forbidden lines in known edge-on disk systems relative to those typically seen in accreting $\mathrm{T}$ Tauri stars.

Another peculiarity of PTF 10nvg is the rare observation of optical TiO/VO molecular bands in emission. Hillenbrand et al. (2012) reported several additional sources besides PTF 10nvg that have been observed to exhibit similar, broad $\mathrm{TiO} / \mathrm{VO}$ emission at optical wavelengths. In common between the sources is the Class I-type SED and spectroscopic evidence for rapid accretion and strong outflow. It is unclear at present whether an "outburst" phase is required for detection of $\mathrm{TiO} / \mathrm{VO}$ emission. Future study of such $\mathrm{TiO} / \mathrm{VO}$ sources may help elucidate the relative molecular abundances in circumstellar disk/envelope environments, and perhaps the mechanisms for molecule formation and destruction. The $\mathrm{TiO}$ and VO species appear from the statistics to be not readily observable during more quiescent stages of disk evolution but notably they are present in planetary atmospheres and so must be present in the disks. Banzatti et al. (2012), for example, have recently discussed in detail for EX Lup the $\mathrm{H}_{2} \mathrm{O}$ and $\mathrm{OH}$ (increased emission during outburst), as well as several organic (decreased emission during outburst) molecules during quiescence and in outburst. These authors discuss the photodissociative and photodesorption effects of enhanced high-energy radiation during an episode of enhanced accretion, and the ensuing broad implications for disk chemistry and structure evolution.

In summary, a coherent physical model for PTF 10nvg would include the following. The optical $\mathrm{TiO} / \mathrm{VO}$ and infrared $\mathrm{CO}$ emission comes from the inner disk region. The broad permitted atomic lines are formed in the accretion/outflow zone, with the narrower atomic lines part of the rest-frame accretion shock. The 2010 brightening was indeed an "outburst" event, driven by accretion, but perhaps accompanied by dust clearing along the line-of-sight. The photometric variability since 2010 is primarily due to large variations in extinction, which periodically obscure the inner disk. This causes the dense gas tracers (molecular $\mathrm{TiO} / \mathrm{VO}, \mathrm{CO}$ and permitted atomic lines) to disappear. However, the low density tracers (molecular $\mathrm{H}_{2}$ and atomic forbidden lines) are coming from an extended jet/outflow, which may include self-shocks. We can see this faint emission only when the central continuum source is obscured. The characteristic timescale of the extinction variations is few-week occultations, with $\sim 221$ day periodicity. If these events are linked with an orbital timescales, it suggests that the occulting material is located at $a \approx 0.7\left(M_{*} / M_{\odot}\right)^{1 / 3} \mathrm{AU}$. The departure from periodic behavior in late 2012 may be due to the dissipation of, or geometrical reconfiguration of, the occulting material. Alternately, it could be due to a recurring dominance of accretion variation behavior over extinction variation behavior, similar to the 2010 outburst that initially drew the attention of several groups worldwide to this source.

\subsection{PTF 10nvg in the Context of Other Large-amplitude Variable Young Stars}

In this section, we discuss the characteristics of PTF 10nvg relative to those of other young stellar objects that have exhibited outbursting behavior, finding some common elements with known classes, but also some unique aspects of PTF 10nvg. We advocate the position that our knowledge of the diversity of time-variable behavior in young stars is vastly incomplete, as is our understanding of the underlying physical mechanisms. Although FU Ori stars are certainly a distinguishable class once the candidates are suitably characterized, other variable categories such as EX Lup-type objects and V1647 Ori-type objects appear to contain a range of behaviors among their members. While all of these variable types are likely explained by time-variable accretion and related outflow, young star "eruptions" may manifest themselves in very different ways with the currently identified categories including somewhat heterogeneous objects at present.

The light curve of PTF 10nvg is dissimilar to those of other categories of young star outbursts. Beyond the initial rise, the 
light curve is very different from those of FU Ori stars, which remain at or near the elevated brightness for decades. The FU Ori stars are inferred to be undergoing significantly enhanced accretion at rates of roughly $10^{-4}$ or $10^{-5} M_{\odot} \mathrm{yr}^{-1}$ (Zhu et al. 2010). The PTF 10nvg light curve is also dissimilar to the light curves of EX Lup-type objects, which have smaller amplitude rises and a clearly distinct quiescent versus outbursting state. The EX Lup star accretion rates are inferred to be lower than those advocated for FU stars; the unusually large 2008 outburst of EX Lup itself was interpreted as an accretion enhancement from roughly $10^{-9} M_{\odot} \mathrm{yr}^{-1}$ to roughly $10^{-7} M_{\odot} \mathrm{yr}^{-1}$ (Sipos et al. 2009; Aspin et al. 2010; Juhász et al. 2012). The light curve for this outburst event (see, e.g., Figure 1 of Juhász et al. 2012) indeed bears some similarity to the photometric behavior of PTF $10 \mathrm{nvg}$, both in terms of the amplitude of the initial rapid rise and the multiple photometric peaks occurring over the next half to three-quarters of a year before the source faded to quiescent levels. As described here, PTF 10nvg also exhibits quasi-periodic brightness peaks at $\sim 7$ month long intervals. However, the outbursting phase with superposed large extinction variations has lasted almost three years now, longer than the events typically associated with EX Lup outbursts. It remains to be seen whether a true quiescent stage will reveal itself in the future.

The physical mechanism for the outbursting behavior in the several classes of large-amplitude variable young stars is thought to be related to an inner disk instability. For FU Ori stars, thermal, gravitational, and magneto-rotational models have all been proposed (Bonnell \& Bastien 1992; Bell \& Lin 1994; Clarke \& Syer 1996; Kley \& Lin 1999; Armitage et al. 2001; Vorobyov \& Basu 2005; Boley et al. 2006; Zhu et al. 2009). For the EX Lup stars, the origin is postulated to be essentially at the inner disk edge itself, and the "instability" driven by a cyclic interaction between the differentially rotating accretion disk and the magnetosphere (roughly dipole in this region), which is rotating with the star. Episodic and sometimes periodic accretion and outflow are naturally explained as a consequence of the oscillations in magnetic field topology (Goodson \& Winglee 1999; Romanova et al. 2005, 2009; D’Angelo \& Spruit 2010, 2012). The field structure alternates between an expanded magnetospheric structure that diffuses into the disk and leads eventually to field line opening and outflow. Following reconnection, a more compact magnetospheric structure results and allows encroachment by the accumulated inner disk material. Enhanced accretion onto the star results when corotation is crossed, after which the magnetosphere again expands. Alternately, the enhanced accretion could involve similar mechanisms but operating in a more stochastic and rapid rather than a semi-cyclic manner (e.g., Romanova et al. 2008). In all of the above scenarios, however, the predicted timescales are highly uncertain, ranging between several times the dynamical timescale and several orders of magnitude times the viscous timescale. Thus it is not yet known if the postulated inner disk oscillations can be associated with the interval between the young star outbursts that have been observed. At least qualitatively, there is some resemblance between the accretion rate evolution during the outbursts described in, e.g., Figure 4 of D'Angelo \& Spruit (2010) and the light curve behavior of PTF 10nvg. The same interpretative conclusion was reached for the 2008 outburst of EX Lup by Juhász et al. (2012).

Covey et al. made the analogy between the outburst behavior of PTF 10nvg and V1647 Ori. This claim was before discovery of the large-amplitude near-infrared variations as consistent with extinction variations, and before discovery of the quasiperiodicity in the light curve. However, Aspin (2011) do claim that the near-infrared color variations of V1647 Ori are consistent with variable extinction (though they argue that those of PTF 10nvg are not consistent with this interpretation, contrary to the evidence presented here). Furthering the analogy, we have found a single outflow cavity in the long-wavelength molecular line observations of PTF 10nvg, which could also be argued for V1647 Ori based on the strong asymmetry in the scattered light nebula.

Lorenzetti et al. (2012) have suggested based on the nearinfrared color differences between the quiescent and the outbursting states of several large-amplitude variables (including PTF 10nvg and V1647 Ori) that the outbursts are characterized by an extra thermal component that is not present during the quiescent stages. In the case of PTF 10nvg, the extra radiation during the outburst is estimated to have $\sim 1400 \mathrm{~K}$ and to be located at $0.3 \mathrm{AU}$ (based on a fitted blackbody model), after accounting for extinction variations of 4.6 mag between a low state and a high state. While our observations indicate that the observed photometric variations (Figure 6) and also spectrophotometric variations (considering a de-reddened version of Figure 11) are roughly consistent with variations in extinction, there is some stochasticity to the color evolution which could indeed necessitate underlying changes in the source SED below the extinction variation which dominates.

If the Lorenzetti et al. (2012) model is robust, the physical situation would be that of changes in thermally emitting material located in the inner region the disk. Although Lorenzetti et al. (2012) interpret this as reprocessing of the radiation emitted on the stellar surface as the accretion shock becomes hotter due to the enhanced accretion rate, one can also draw an analogy to the above theories invoking inner disk instabilities wherein the inner disk heating is due to viscous and dynamical process rather than radiative reprocessing. Notably, the $\sim 0.3 \mathrm{AU}$ scale derived in the Lorenzetti et al. (2012) blackbody model is not so different from the $a \approx 0.7\left(M_{*} / M_{\odot}\right)^{1 / 3}$ AU scale that was found above in our discussion of the semi-periodicity of the repeated photometric peaks. However, perhaps inconsistent between the postulation of an extra, cool emission component and the instability theory, is that there does not appear to be any lag between the infrared brightening and the optical brightening in PTF 10nvg. This is true for the initially recorded outburst in 2010 as well as all subsequent brightenings that we have observed from 2010 to 2012. Lorenzetti et al. (2012) do note that the blackbody model becomes less good of a fit as their EX Lup-type sources evolve beyond the initial photometric outburst toward quiescence.

\subsection{The Rate of PTF 10nvg-like Events}

As detailed both here and in previous work (Covey et al. 2011; Aspin 2011), PTF 10nvg does not belong to either the FU Orilike or the EX Lup-like classes of young stellar object outbursts. It may be an intermediate timescale and amplitude outburst class, perhaps analogous to V1647 Ori as discussed in Covey et al. Or it may be a more extreme version of the extinctiondriven phenomena seen in UX Ori or GM Cep. We advocate that both phenomena (accretion and extinction) play some role in the interpretation of PTF 10nvg photometry and spectroscopy. At present, estimates of the absolute rates of these types of largeamplitude events (whether accretion-driven, extinction-driven, or both) remain highly uncertain. Nevertheless, we attempt a rough estimate from our ongoing photometric monitoring survey 
of the North America Nebula of the relative rate of PTF 10nvgtype phenomena.

To our knowledge, the P48 survey of the North America Nebula region is unique in both its depth and cadence. During the past $\sim 3$ years the P48 has observed the same $\sim 7.3 \mathrm{deg}^{2}$ field with roughly a nightly cadence while it was visible from Palomar mountain to a depth of $\sim 20$ mag per observation. In this time there has been one FU Ori-like outburst (HBC 722/PTF 10qpf; Semkov et al. 2010; Miller et al. 2011) and one PTF 10nvg-like outburst event, suggesting that the rate of the two phenomena is similar to within the accuracy afforded by low number statistics. A more general discussion of smalleramplitude but significant outbursting and extinction dominated behavior among young members of the North America Nebula will be presented by Findeisen et al. (2013).

Overall, with $\sim 12$ FU Ori-like eruptions observed to date (Reipurth \& Aspin 2010) and only one PTF 10nvg-like event it is also possible that PTF 10nvg-like events are far more rare and only $\sim$ one-tenth as common as FU Ori outbursts. With $\sim 10$ FU Ori-like outbursts expected for each low mass star (Hartmann \& Kenyon 1996), even this lower limit would suggest 1 PTF 10nvg-like outburst per low mass star. Likewise, in the interpretation that this event corresponds to the initial cloud clearing and source revelation, we would expect $\sim 1$ event per star.

As future surveys continue to study star forming regions with high cadence observations over long time baselines, the importance of PTF 10nvg-like events in our global understanding of the low mass star formation process will be elucidated. History shows that astronomy is rich with examples of new phenomena being discovered as surveys push to explore undersampled regions of parameter space.

\section{SUMMARY AND CONCLUSIONS}

1. Continued photometric monitoring of PTF 10nvg by the P48 and PAIRITEL telescopes throughout 2011 and 2012 detected magnitude variations of $\Delta R \sim 10 \mathrm{mag}$ and $\Delta K \sim 3 \mathrm{mag}$, and color variations of $\Delta J-K \sim 3 \mathrm{mag}$. The time-series behavior in color-color and color-magnitude diagrams indicates that these photometric variations are consistent with those expected due to changes in line-ofsight extinction amounting to $\Delta A_{V}>30 \mathrm{mag}$.

2. Over the long term, attributing PTF 10nvg's non-detection in 2MASS to similar extinction changes suggests the source has historically experienced a range of $\Delta A_{V} \sim 70 \mathrm{mag}$. Alternately, the non-detection in $2 \mathrm{MASS}$ may reflect a true outburst scenario for the 2010 brightening and subsequent photometric evolution.

3. Timescales of several weeks can be associated with dramatic flare-like photometric changes and $\sim 7$ months with the repeated maxima observed in the time series over the past several years. While the initial 2010 maxima were rounded/smooth, later maxima in 2011 and early 2012 were characterized by narrow/sharp photometric peaks. A late2012 maximum was again broad and rounded/smooth.

4. The Lomb-Scargle periodogram calculated from $J H K_{s}$ light curves between 2010 and mid-2012 features a prominent, statistically significant peak at $\sim 221$ days. Under the assumption that the period is the Keplerian orbital timescale, the dust obscuration governing PTF 10nvg's photometric behavior is located at about $0.7\left(M_{*} / M_{\odot}\right)^{1 / 3} \mathrm{AU}$ from the central star. Continued monitoring will be essen- tial to confirm the reality of the periodic signal; the existing light curves span $<1000$ days in total, and include several $100+$ day gaps. Further, the late-2012 light curve appears to depart from the previously derived periodic trend and may reflect renewed dominance of accretion over extinction effects.

5. WISE detected PTF 10nvg over multiple epochs in 2010. Comparison of these mid-infrared detections with prior detections at similar wavelengths by IRAS, Spitzer, and $M S X$ indicates that PTF 10nvg brightened in the midinfrared by factors of a few during its summer 2010 brightening.

6. CARMA observations reveal an extended millimeter continuum source having a total (dust plus gas) mass of $0.06 M_{\odot}$. CARMA also detects spatially unresolved emission in both ${ }^{12} \mathrm{CO}$ and ${ }^{13} \mathrm{CO}$ centered on PTF 10 nvg's nearinfrared position and systemic velocity. Spatially extended, redshifted ${ }^{12} \mathrm{CO}$ emission is detected to the south of PTF $10 \mathrm{nvg}$, which we interpret as revealing the presence of an outflow cavity. Over the wider field, the molecular emission is well aligned with the $\mathrm{H} \alpha$ emission arc.

7. The bright-state optical and near-infrared spectrum of PTF 10 nvg is that of a "continuum-plus-emission" object, similar to V1331 Cyg but with significant molecular emission contributions to the continuum. The faint-state spectrum is that of a Herbig-Haro object. Both neutral and singly ionized line species probing a range of densities and temperatures are observed. The lines that characterize PTF 10nvg's faint-state spectrum are present at all epochs, however, suggesting that the spectral evolution is primarily due to the suppression of the bright-state spectral features during epochs of enhanced extinction.

8. Spectral monitoring reveals that the strong wind signatures detected in the 2010 outburst persisted through 2011 and 2012. The depth and terminal velocities of optical wind absorption features (i.e., the Na I D and KI 7665/7699 doublets, the OI 7774 triplet) appeared consistent-or perhaps slightly increased over time in some lines-across all epochs where the optical continuum was sufficiently strong to enable these features to be measured. In the nearinfrared, He I $\lambda 10830$ absorption increased in both depth and equivalent width by nearly a factor of two from 2010 through 2011. A strong redshifted emission component to He I $\lambda 10830$ likely also traces the outflow rate of the inner wind.

9. The evolution of the strengths and profiles of permitted atomic emission lines reveal the presence of time variability in PTF 10nvg's line-of-sight accretion and outflow activity. The source exhibits optically thick Ca II triplet emission with line ratios that are unusual even for young stars. The $\mathrm{H} \alpha$ emission also demonstrates significant variations in both overall strength and kinematic profile, with the line weakening and shifting blueward as the source fades. Strong redshifted $\mathrm{H} \alpha$ emission disappeared in June 2011 as PTF 10nvg approached its photometric minimum, while the blueshifted $\mathrm{H} \alpha$ emission remained distinctly visible. These observations suggest that PTF 10nvg's Ca II and H I lines include contributions from both accretion and outflow activity. While the accretion component may dominate during the brightest states, during fainter states only the outflow signature remains. Notably absent from the spectra at any epoch is the $\mathrm{He} \mathrm{I} \lambda 5876$ line, a high-temperature 
$(T>15,000 \mathrm{~K})$ tracer seen near-ubiquitously in classical $\mathrm{T}$ Tauri stars.

10. Forbidden line emission measuring 5000-20,0000 K gas reveals the presence of a likely Herbig-Haro jet with a wide range of densities, $n \sim 10^{3}-10^{7} \mathrm{~cm}^{-3}$. Some line ratios such as [O I] $6300 \AA: 6363 \AA$ and [Fe II] $7155 \AA: 7172 \AA$ show extreme and unusually time-dependent ratios that are not easily explained in the standard optically thin assumption for forbidden line emission. Instead, these lines may be partially optically thick.

11. Spatially extended blueshifted emission is visible in several forbidden line species as well as $\mathrm{H} \alpha$ in two-dimensional HIRES images. These position-velocity diagrams provide further evidence that PTF 10nvg is driving a jet with a line-of-sight velocity of about $-200 \mathrm{~km} \mathrm{~s}^{-1}$ and exciting a strong shock region $\sim 4^{\prime \prime}$ projected distance from the source (2000AU at $520 \mathrm{pc}$ ).

12. The moderate resolution near-infrared spectra obtained in 2011 June/July, when PTF 10nvg was near its faintest state, provide an opportunity to study the properties of the jet and extended outflow. PTF 10nvg's $\mathrm{H}_{2}$ line strengths strongly resemble theoretical predictions for $\mathrm{C}$-type shocks and pure $\mathrm{X}$-ray photoexcitation, as well as empirical observations of $\mathrm{H}_{2}$ emission from RW Aur and Knot B of the HH54 protostellar jet; the same is true for the near-infrared [Fe II] emission.

13. From near-infrared adaptive optics observations we can exclude the presence of stellar companions within several hundred AU of PTF 10nvg.

Overall, we conclude that the recent time-series data on PTF 10nvg will not be easy to interpret without a good deal of hindsight. Further observational and theoretical study of this enigmatic source is warranted.

We commend PTF and PAIRITEL critical personnel Peter Nugent, Eran Ofek, and Dan Starr, and Cullen Blake for effective facility operation and data distribution. Mark Sullivan ran his PTF-specific PSF fitting code for us. In addition, we thank Joey Richards for helpful conversations and advice about the light curve analysis. We are also grateful to the many observers who kindly helped with the acquisition and/or reduction of spectra that are reported either in this paper or in Covey et al. (2011), including Katie Hamren, Everett Schlwain, Aaron Barth, Evan Kirby, Melissa Graham, Eric Hsiao, Peter Blanchard, Antonio Cucchiara, Adam Morgan, Daniel Perley, Christopher Griffith, Michael Kandrashoff, Luisa Rebull, Wilson Liu, John Angione, Shriharsh Tendulkar, Aaron Ofer, Kelsey Clubb, Greg Herczeg, Adam Kraus, Nick Law, Andrew Howard, Dan DeFelippis, Kunal Mooley, Trevor David, Geoff Marcy, and Howard Isaacson. CARMA Summer School students Isaac Shivvers, Che-Yu Chen, and Aaron Juarez participated in some of the CARMA observations and data reduction described herein. We are also very grateful for the assistance of staff members at the observatories where data were obtained. Funding acknowledgements are as follows. (1) K.R.C. acknowledges support for this work from the Hubble Fellowship Program, provided by NASA through Hubble Fellowship grant HST-HF-51253.01-A awarded by the STScI, which is operated by the AURA, Inc., for NASA, under contract NAS 5-26555, and support for publication charges generously provided by Dr. William Tifft. (2) A.V.F.'s group at UC Berkeley acknowledges generous financial assistance from Gary and Cynthia Bengier, the Christopher R. Redlich Fund, the Richard and Rhoda Goldman Fund, the TABASGO Foundation, and US National Science Foundation (NSF) grant AST0908886. (3) PAIRITEL has been supported by a Swift Guest Investigator grant NASA/NNX12AE67G. (4) Some of the data presented herein were obtained at the W. M. Keck Observatory, which is operated as a scientific partnership among the California Institute of Technology, the University of California, and NASA; the observatory was made possible by the generous financial support of the W. M. Keck Foundation. (5) The Infrared Telescope Facility is operated by the University of Hawaii under Cooperative Agreement No. NNX-08AE38A with the National Aeronautics and Space Administration, Science Mission Directorate, Planetary Astronomy Program. (6) Support for CARMA construction was derived from the Gordon and Betty Moore Foundation, the Kenneth T. and Eileen L. Norris Foundation, the James S. McDonnell Foundation, the Associates of the California Institute of Technology, the University of Chicago, the states of California, Illinois, and Maryland, and the National Science Foundation. Ongoing CARMA development and operations are supported by the National Science Foundation under a cooperative agreement (grant number AST-0838260) and by the CARMA partner universities.

Facilities: PO:1.2m, FLWO:2MASS, Shane, Hale (two instruments), ARC, Keck:I, Keck:II, IRTF, CARMA, WIYN:0.9m, UKIRT, Spitzer, WISE

\section{REFERENCES}

Appenzeller, I., Bertout, C., \& Stahl, O. 2005, A\&A, 434, 1005 Armitage, P. J., Livio, M., \& Pringle, J. E. 2001, MNRAS, 324, 705 Aspin, C. 2011, AJ, 141, 196

Aspin, C., Reipurth, B., Herczeg, G. J., \& Capak, P. 2010, ApJL, 719, L50

Banzatti, A., Meyer, M. R., Bruderer, S., et al. 2012, ApJ, 745, 90

Bautista, M. A., Peng, J., \& Pradhan, A. K. 1996, ApJ, 460, 372

Bautista, M. A., \& Pradhan, A. K. 1998, ApJ, 492, 650

Beck, T. L., McGregor, P. J., Takami, M., \& Pyo, T.-S. 2008, ApJ, 676, 472

Beckwith, S. V. W., Sargent, A. I., Chini, R. S., \& Guesten, R. 1990, AJ, 99, 924

Bell, K. R., \& Lin, D. N. C. 1994, ApJ, 427, 987

Bertin, E., \& Arnouts, S. 1996, A\&AS, 117, 393

Blake, C. H., Bloom, J. S., Latham, D. W., et al. 2008, PASP, 120, 860

Bloom, J. S., Perley, D. A., Li, W., et al. 2009, ApJ, 691, 723

Bloom, J. S., Richards, J. W., Nugent, P. E., et al. 2012, PASP, 124, 1175

Bloom, J. S., Starr, D. L., Blake, C. H., Skrutskie, M. F., \& Falco, E. E. 2006, in ASP Conf. Ser. 351, Astronomical Data Analysis Software and Systems XV, ed. C. Gabriel, C. Arviset, D. Ponz, \& S. Enrique (San Francisco, CA: ASP), 751

Boley, A. C., Mejía, A. C., Durisen, R. H., et al. 2006, ApJ, 651, 517

Bonnell, I., \& Bastien, P. 1992, ApJL, 401, L31

Brugel, E. W., Boehm, K. H., \& Mannery, E. 1981, ApJS, 47, 117

Carpenter, J. M. 2001, AJ, 121, 2851

Chen, W. P., Hu, S. C.-L., Errmann, R., et al. 2012, ApJ, 751, 118

Clarke, C. J., \& Syer, D. 1996, MNRAS, 278, L23

Clayton, G. C. 2012, JAVSO, 40, 539

Cody, A. M., \& Hillenbrand, L. A. 2010, ApJS, 191, 389

Cody, A. M., \& Hillenbrand, L. A. 2011, ApJ, 741, 9

Connelley, M. S., \& Greene, T. P. 2010, AJ, 140, 1214

Covey, K. R., Hillenbrand, L. A., Miller, A. A., et al. 2011, AJ, 141, 40

D’Angelo, C. R., \& Spruit, H. C. 2010, MNRAS, 406, 1208

D’Angelo, C. R., \& Spruit, H. C. 2012, MNRAS, 420, 416

Doppmann, G. W., Greene, T. P., Covey, K. R., \& Lada, C. J. 2005, AJ, 130, 1145

Dougados, C., Bacciotti, F., Cabrit, S., \& Nisini, B. 2010, Jets from Young Stars IV (Lecture Notes in Physics, Vol. 793; Springer: Berlin), 213

Edwards, S., Fischer, W., Hillenbrand, L., \& Kwan, J. 2006, ApJ, 646, 319

Edwards, S., Fischer, W., Kwan, J., Hillenbrand, L., \& Dupree, A. K. 2003, ApJL, 599, L41

Espaillat, C., Furlan, E., D’Alessio, P., et al. 2011, ApJ, 728, 49

Findeisen, K., Hillenbrand, L., Ofek, E., et al. 2013, ApJ submitted

Fitzpatrick, E. L. 1999, PASP, 111, 63

Flaherty, K. M., Muzerolle, J., Rieke, G., et al. 2012, ApJ, 748, 71 
Giannini, T., McCoey, C., Nisini, B., et al. 2006, A\&A, 459, 821

Girardi, L., Bertelli, G., Bressan, A., et al. 2002, A\&A, 391, 195

Goodson, A. P., \& Winglee, R. M. 1999, ApJ, 524, 159

Greene, T. P., Barsony, M., \& Weintraub, D. A. 2010, ApJ, 725, 1100

Hamann, F., \& Persson, S. E. 1989, ApJ, 339, 1078

Hamilton, C. M., Johns-Krull, C. M., Mundt, R., Herbst, W., \& Winn, J. N. 2012, ApJ, 751, 147

Hartigan, P., Bally, J., Reipurth, B., \& Morse, J. A. 2000, Protostars and Planets IV (Tucson, AZ: Univ. Arizona Press), 841

Hartmann, L., \& Kenyon, S. J. 1996, ARA\&A, 34, 207

Herter, T. L., Henderson, C. P., Wilson, J. C., et al. 2008, Proc. SPIE, 7014, $70140 \mathrm{X}$

Hillenbrand, L. A. 1997, AJ, 113, 1733

Hillenbrand, L. A., Knapp, G. R., Padgett, D. L., Rebull, L. M., \& McGehee, P. M. 2012, AJ, 143, 37

Hinkley, S., Rice, E., Hillenbrand, L., et al. 2013, ApJL, 763, L9

Indebetouw, R., Mathis, J. S., Babler, B. L., et al. 2005, ApJ, 619, 931

Isella, A., Pérez, L. M., \& Carpenter, J. M. 2012, ApJ, 747, 136

Jørgensen, J. K., Hogerheijde, M. R., van Dishoeck, E. F., Blake, G. A., \& Schöier, F. L. 2004, A\&A, 413, 993

Juhász, A., Dullemond, C. P., van Boekel, R., et al. 2012, ApJ, 744, 118

Kley, W., \& Lin, D. N. C. 1999, ApJ, 518, 833

Koresko, C. D., Beckwith, S. V. W., Ghez, A. M., Matthews, K., \& Neugebauer, G. 1991, AJ, 102, 2073

Kóspál, Á., Ábrahám, P., Acosta-Pulido, J. A., et al. 2011, A\&A, 527, A133

Kurosawa, R., \& Romanova, M. M. 2012, MNRAS, 426, 2901

Kwan, J., \& Fischer, W. 2011, MNRAS, 411, 2383

Lada, C. J. 1987, Star Forming Regions (Dordrecht: Reidel), 1

Law, N. M., Kulkarni, S. R., Dekany, R. G., et al. 2009, PASP, 121, 1395

Li, H., \& McCray, R. 1992, ApJ, 387, 309

Lomb, N. R. 1976, Ap\&SS, 39, 447

Lorenzetti, D., Antoniucci, S., Giannini, T., et al. 2012, ApJ, 749, 188

McCarthy, J. K., et al. 1998, Proc. SPIE, 3355, 81

McLean, I. S., Becklin, E. E., Bendiksen, O., et al. 1998, Proc. SPIE, 3354, 566

Meyer, M. R., Calvet, N., \& Hillenbrand, L. A. 1997, AJ, 114, 288

Miller, A. A., Hillenbrand, L. A., Covey, K. R., et al. 2011, ApJ, 730, 80

Miller, J. S., \& Stone, R. P. S. 1993, Lick Obs. Tech. Rep. 66 (Santa Cruz: Lick Obs.)

Morales-Calderón, M., Stauffer, J. R., Hillenbrand, L. A., et al. 2011, ApJ, 733,50

Muzerolle, J., Flaherty, K., Balog, Z., et al. 2009, ApJL, 704, L15

Muzerolle, J., Hartmann, L., \& Calvet, N. 1998, AJ, 116, 2965

Najita, J., Carr, J. S., Glassgold, A. E., Shu, F. H., \& Tokunaga, A. T. 1996, ApJ, 462, 919

Nomura, H., Aikawa, Y., Tsujimoto, M., Nakagawa, Y., \& Millar, T. J. 2007, ApJ, 661,334

Ofek, E. O., Laher, R., Law, N., et al. 2012, PASP, 124, 62
Oke, J. B., Cohen, J. G., Carr, M., et al. 1995, PASP, 107, 375

Oke, J. B., \& Gunn, J. E. 1982, PASP, 94, 586

Perley, D. A., Bloom, J. S., Klein, C. R., et al. 2010, MNRAS, 406, 2473

Plavchan, P., Gee, A. H., Stapelfeldt, K., \& Becker, A. 2008, ApJL, 684, L37

Plavchan, P., Laohakunakorn, N., Seifahrt, A., Staplefeldt, K., \& Gee, A. H. 2010, BAAS, 42, 350

Pueyo, L., Hillenbrand, L., Gautam, V., et al. 2012, ApJ, 757, 57

Ralchenko, Yu., Kramida, A. E., Reader, J., \& NIST ASD Team 2011, NIST Atomic Spectra Database, ver. 4.1.0 (Gaithersburg, MD: National Institute of Standards and Technology), available online at http://physics.nist.gov/asd

Rau, A., Kulkarni, S. R., Law, N. M., et al. 2009, PASP, 121, 1334

Rayner, J. T., Toomey, D. W., Onaka, P. M., et al. 2003, PASP, 115, 362

Rebull, L. M., Guieu, S., Stauffer, J. R., et al. 2011, ApJS, 193, 25

Reipurth, B., \& Aspin, C. 2010, in Evolution of Cosmic Objects through Their Physical Activity, ed. H. A. Harutyunian, A. M. Mickaelian, \& Y. Terzian (Yerevan: Gitutyun Publ. House of NAS RA), 19

Richards, J. W., Starr, D. L., Butler, N. R., et al. 2011, ApJ, 733, 10

Romanova, M. M., Kulkarni, A. K., \& Lovelace, R. V. E. 2008, ApJL, 673, L171

Romanova, M. M., Ustyugova, G. V., Koldoba, A. V., \& Lovelace, R. V. E. 2005, ApJL, 635, L165

Romanova, M. M., Ustyugova, G. V., Koldoba, A. V., \& Lovelace, R. V. E. 2009, MNRAS, 399, 1802

Rosolowsky, E., Dunham, M. K., Ginsburg, A., et al. 2010, ApJS, 188, 123

Scargle, J. D. 1982, ApJ, 263, 835

Semkov, E. H., \& Peneva, S. P. 2012, Ap\&SS, 338, 95

Semkov, E. H., Peneva, S. P., Munari, U., Milani, A., \& Valisa, P. 2010, A\&A, $523, \mathrm{~L} 3$

Sicilia-Aguilar, A., Merín, B., Hormuth, F., et al. 2008, ApJ, 673, 382

Simon, T., \& Joyce, R. R. 1988, PASP, 100, 1549

Sipos, N., Ábrahám, P., Acosta-Pulido, J., et al. 2009, A\&A, 507, 881

Smith, M. D. 1995, A\&A, 296, 789

Steidel, C. C., Shapley, A. E., Pettini, M., et al. 2004, ApJ, 604, 534

Sullivan, M., Kasliwal, M. M., Nugent, P. E., et al. 2011, ApJ, 732, 118

van Boekel, R., Juhász, A., Henning, T., et al. 2010, A\&A, 517, A16

Vogt, S. S., Allen, S. L., Bigelow, B. C., et al. 1994, Proc. SPIE, 2198, 362

Vorobyov, E. I., \& Basu, S. 2005, ApJL, 633, L137

Wang, H., Apai, D., Henning, T., \& Pascucci, I. 2004, ApJL, 601, L83

White, R. J., \& Hillenbrand, L. A. 2004, ApJ, 616, 998

Wilson, J. C., Henderson, C. P., Herter, T. L., et al. 2004, Proc. SPIE, 5492, 1295

Xiao, L., Kroll, P., \& Henden, A. A. 2010, AJ, 139, 1527

Zechmeister, M., \& Kürster, M. 2009, A\&A, 496, 577

Zhu, Z., Hartmann, L., Calvet, N., et al. 2008, ApJ, 684, 1281

Zhu, Z., Hartmann, L., Gammie, C., \& McKinney, J. C. 2009, ApJ, 701, 620

Zhu, Z., Hartmann, L., Gammie, C. F., et al. 2010, ApJ, 713, 1134 